\title{
Ingestion Pathway Consequences of an H-Separations Coil and Tube Failure
}

by

A. Blanchard

Westinghouse Savannah River Company

Savannah River Site

Aiken, South Carolina 29808

J. Thompson

Westinghouse Safety Management Solutions
TECEIVED

AUG 161999

OSTI

This paper was prepared in connection with work done under the above contract number with the U.S. Department of Energy. By acceptance of this paper, the publisher and/or recipient acknowledges the U.S. Government's right to retain a nonexclusive, royalty-free license in and to any copyright covering this paper, along with the right to reproduce and to authorize others to reproduce all or part of the copyrighted paper. 
Keywords: Ingestion Pathways Emergency Planning Food Interdiction

Retention: Lifetime

\section{Ingestion Pathway Consequences of an H-Separations Coil and Tube Failure (U)}

Jay M. Thompson

April 1999

Classification

\section{UNCLASSIFIED}

DOES NOT CONTAIN

UNCLASSIFIED CONTROLLED

NUCLEAR INFORMATION

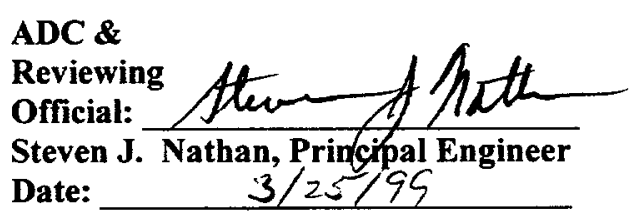

Date:

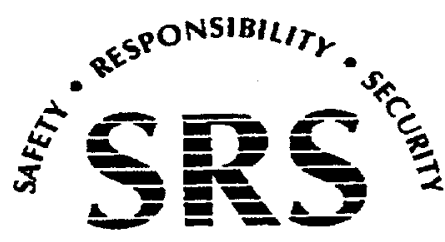

SAVANNAH RIVER SITE

Westinghouse Savannah River Company Technical Services Division Aiken, SC 29808 
Ingestion Pathway Consequences of an H-Separations Coil and Tube Failure (U)

Jay M. Thompson

April 1999

Westinghouse Savannah River Company Technical Services Division

Aiken, SC 29808

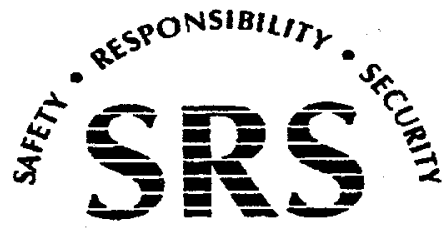

SAVANNAH RIVER SITE

PREPARED FOR THE U.S. DEPARTMENT OF ENERGY UNDER CONTRACT NO. DE-AC09-96SR18500 


\section{DISCLAIMER}

This report was prepared as an account of work sponsored by an agency of the United States Government. Neither the United States Government nor any agency thereof, nor any of their employees, makes any warranty, express or implied, or assumes any legal liability or responsibility for the accuracy, completeness, or usefulness of any information, apparatus, product, or process disclosed, or represents that its use would not infringe privately owned rights. Reference herein to any specific commercial product, process, or service by trade name, trademark, manufacturer, or otherwise does not necessarily constitute or imply its endorsement, recommendation, or favoring by the United States Government or any agency thereof. The views and opinions of authors expressed herein do not necessarily state or reflect those of the United States Government or any agency thereof.

This report has been reproduced directly from the best available copy.

Available to DOE and DOE contractors from the Office of Scientific and Technical Information, P.O. Box 62, Oak Ridge, TN 37831; prices available from (615) 576-8401.

Available to the public from the National Technical Information Service, U.S. Department of Commerce; 5285 Port Royal Road, Springfield, VA 22161. 


\section{DISCLAIMER}

\section{Portions of this document may be illegible in electronic image products. Images are produced from the best available original document.}


PROJECT: $\quad$ N/A

DOCUMENT:

TITLE:
WSRC-TR-99-00089

Ingestion Pathway Consequences of an $\mathrm{H}$-Separations Coil and Tube Failure (U)

REVISION NUMBER:

0

AUTHOR/TECHNICAL REVIEWS:

Inn Tihomesen

J. M. Thompson, Author

C.Q. Othonnower

E. A. Thompson, Technical Reviewer $\frac{3 / 25 / 99}{\text { Date }}$

$4 / 6 / 99$

Date
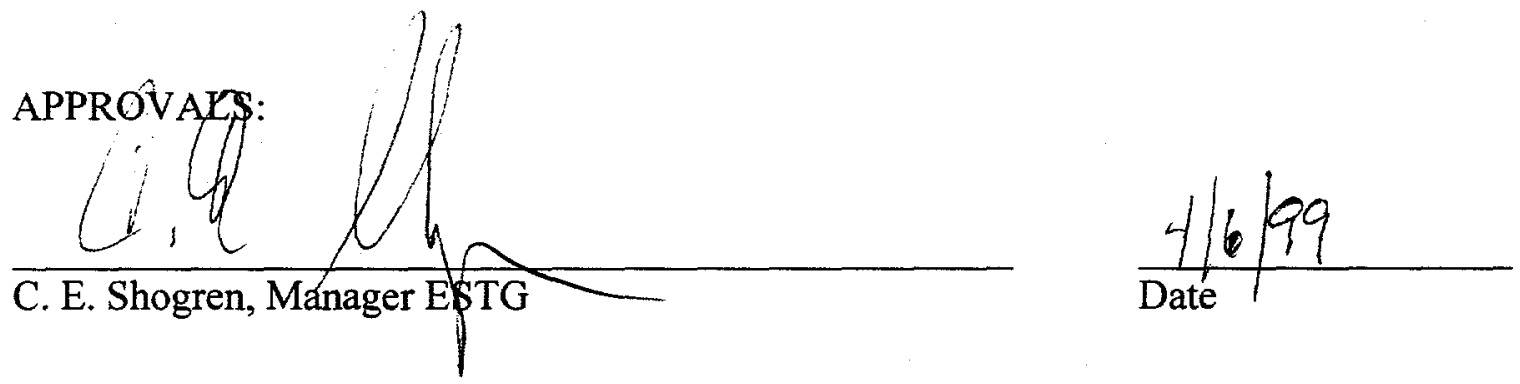

Za What

M. J. Hitchler, Principal, Safety Analysis Services

$\frac{417 / 95}{\text { Date }}$ 


\begin{abstract}
The food ingestion consequences of an accidental release, scenario 1-RD-2.35, are evaluated for H-Separations. The sizes of land areas requiring the protective action of food interdiction are calculated. The consequences of the release are evaluated with the HOTSPOT model and an EXCEL spreadsheet.
\end{abstract}




\section{CONTENTS}

Introduction
Approach
Background
Analysis
Results
Conclusion
Attachments

\section{TABLES}

Table 1. H-Separations airborne source term, 1-RD-2.35 ........................................... 2

Table 2. Areal concentrations for a unit deposition ........................................................... 3

Table 3. Radionuclide concentration in milk per unit deposition of particulate mix ......... 3

Table 4. Derived response levels for the milk pathway .................................................... 4

Table 5. Derived response levels for all pathways ................................................................ 4

Table 6 Food pathways ranked by DRL …………………........................................... 5

Table 7. HOTSPOT Parameters used for the H-Separations Coil \& Tube release $(1-R D-2.35)$

Table 8. Ranges until deposition less than DRL, average meteorology, H-Separations, $1-\mathrm{RD}-2.35$.

Table 9. Areas above DRL, average meteorology, H-Separations, 1-RD-2.35 ................. 7

Table 10. Ranges until deposition less than DRL, 95\% adverse meteorology,

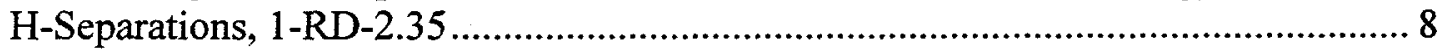

Table 11. Areas above DRL, 95\% adverse meteorology, H-Separations, 1-RD-2.35 ....... 8 


\section{Ingestion Pathway Consequences of an.H-Separations Coil and Tube Failure (U)}

\section{Introduction}

Accidental releases of radionuclides from nuclear facilities may cause contamination of food and forage. Numerous pathways exist for deposited radionuclides to reach man. The major pathways considered by recent Food and Drug Administration guidance are analyzed in this report.

\section{Approach}

The general methodology for determining the limiting radionuclide group, Derived Response Level (DRL), and consequences for ingestion pathways is outlined below.

1. Determine the source terms (radionuclide-specific and total airborne releases).

2. If tritium is present in the source term, evaluate the consequences of the tritium release using UFOTRI. Tritium is not present in the source term of scenario 1-RD2.35 .

3. Calculate the concentrations of each radionuclide in foodstuffs for a unit deposition of the particulate mix.

4. For radionuclides with FDA (1998) Derived Intervention Levels (DILs), divide the DIL by the foodstuff concentration from a unit deposition. The Derived Response Level, or DRL, is the deposition that gives a DIL for a specific nuclide (group) and pathway.

5. Run the dispersion/deposition model HOTSPOT to determine areas impacted.

\section{Background}

FDA (1998) places selected radionuclides into five groups. These groups are:

- $\operatorname{Sr}-90$

- I-131

- Cs-134+ Cs-137

- $\mathrm{Pu}-238+\mathrm{Pu}-239+\mathrm{Am}-241$

- $\mathrm{Ru}-103+\mathrm{Ru}-106$

Notes for Table 2 and Appendix D of FDA (1998) provide guidance on how to utilize these groups. The Derived Intervention Level (DIL) for each radionuclide group is applied independently (i.e., there is no additivity between groups). If multiple radionuclides are present in a group, the DIL applies to the sum of the concentrations of 
those radionuclides. The DILs are applied to the wet weight of foods (as prepared for consumption).

These groups were developed to aid analyses at a variety of nuclear facilities. Nuclear waste storage facilities and nuclear fuel reprocessing facilities are expected to have $\mathrm{Sr}-90$, Cs-137, Pu-239, and Pu-238 as major contributors (p. 51 of FDA 1998).

FDA recommends that DILs from the five groups be applied immediately following an accident. Early evaluation of other radionuclides that may have been released is not required. However, an evaluation should be performed as soon as possible to ensure the DILs are appropriate; presumably, this would include evaluating DILs for the five groups as well as those for radionuclides not in the groups.

The five radionuclide groups are not sufficient to cover accidents at all types of facilities or locations. FDA (1998) notes the example of transportation accidents that may release radionuclides not in the radionuclide groups. Although such releases are not specifically addressed (in the five groups), FDA recommends that an evaluation of the radiation dose from ingestion of these other radionuclides be performed to determine if protective action guides will be exceeded. Appendix E of FDA (1998) presents DILs for 15 additional radionuclides.

Some source terms at SRS are postulated to be dominated by tritiated water vapor. DILs for tritium in water vapor and organically bound forms were derived in WSRC-TR-99-00064 using methodology consistent with FDA (1998). The tritiumspecific model UFOTRI is used to evaluate tritium releases. Tritium is not present in the source term of scenario 1-RD-2.35.

\section{Analysis}

For H-Separations, the source term for the Coil \& Tube Release Scenario 1-RD-2.35 is evaluated. Respirable releases are the same as total releases for each radionuclide since the respirable fraction (RF) is 1.0 (S-CLC-G-00004, Sheet 6, section 3.2). S-CLC-G-00004 contains the source term calculations used ultimately in S-EHA-H-00004. The source terms are listed in Table 1.

Table 1. H-Separations airborne source term, 1-RD-2.35

\begin{tabular}{lcc}
\hline Nuclide & & Airborne Release (Ci) \\
\hline $\mathrm{Am}$ & 241 & $6.57 \mathrm{E}-01$ \\
$\mathrm{Pu}$ & 238 & $1.62 \mathrm{E}+00$ \\
$\mathrm{Pu}$ & 239 & $4.23 \mathrm{E}-03$ \\
$\mathrm{Pu}$ & 240 & $2.43 \mathrm{E}-01$ \\
$\mathrm{Pu}$ & 241 & $3.26 \mathrm{E}+01$ \\
$\mathrm{Pu}$ & 242 & $1.54 \mathrm{E}-02$ \\
Total & & $3.51 \mathrm{E}+01$ \\
\hline
\end{tabular}

Reference: Source_H.xls, Worksheet "Quantities", column G (included in Attachment A of this report); respirable release from S-EHA-H-00004, Rev. 1, Appendix B, Calculation 3, Sheet 6. 


\section{Classification of release into FDA radionuclide groups}

The $\mathrm{Pu}-238+\mathrm{Pu}-239+\mathrm{Am}-241$ group is the only one of the five radionuclide groups that is represented. The only one of the fifteen additional radionuclides evaluated in FDA (1998) that is in this source term is $\mathrm{Pu}-241$. Pu-241 has a DIL of $120 \mathrm{~Bq} \mathrm{~kg}^{-1}$. DILs for $\mathrm{Pu}-240$ and $\mathrm{Pu}-242$ are not given in FDA (1998) and are not evaluated in this report.

\section{Evaluation of the deposition needed to exceed a DIL}

Assume a deposition of $1 \mathrm{Ci} \mathrm{m}^{-2}$ of the nuclide mix, and that all radionuclides deposit similarly. In this case, the relative concentrations of the deposited material will be the same as that released. The quantities of the individual radionuclides present for this unit deposition are given in Table 2. Attachment A presents applicable spreadsheet calculations.

Table 2. Areal concentrations for a unit deposition

\begin{tabular}{cc}
\hline Nuclide & Areal concentration per unit deposition $\left(\mathrm{Ci} \mathrm{m}^{-2}\right)$ \\
\hline Am241 & $1.87 \mathrm{E}-02$ \\
Pu238 & $4.61 \mathrm{E}-02$ \\
Pu239 & $1.20 \mathrm{E}-04$ \\
Pu240 & $6.92 \mathrm{E}-03$ \\
Pu241 & $9.28 \mathrm{E}-01$ \\
Pu242 & $4.38 \mathrm{E}-04$ \\
\hline Reference: Source H.xls, Worksheet "Quantities", column I
\end{tabular}

The concentrations in various foodstuffs due to this unit deposition may now be evaluated. The concentration of a particular radionuclide in a foodstuff is equal to the deposition times the (radionuclide-specific) overall transfer factor. Division of the DIL by the concentration in the foodstuff resulting from a unit deposition gives the deposition needed for a DIL. This process is presented in Tables 3 and 4 for the dairy pathway. Attachment B presents applicable spreadsheet calculations.

Transfer factors for plutonium and americium are derived in WSRC-TR-99-00005, Ingestion Pathway Transfer Factors for Plutonium and Americium. These two elements encompass all radionuclides released in scenario 1-RD-2.35.

Table 3. Radionuclide concentration in milk per unit deposition of particulate mix

\begin{tabular}{|c|c|c|c|c|c|}
\hline Nuclide & 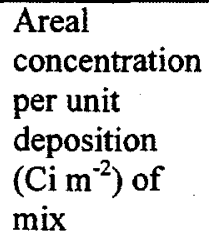 & $\begin{array}{l}\text { Overall } \\
\text { transfer factor } \\
\mathrm{T}_{\text {milk }}\left(\mathrm{m}^{2} \mathrm{~L}^{-1}\right)\end{array}$ & $\begin{array}{l}\text { Concentration } \\
\text { in milk }\left(\mathrm{Ci}^{-1}\right) \\
\text { per unit } \\
\text { deposition }\end{array}$ & $\begin{array}{l}\text { Radionuclide } \\
\text { Group } \\
\text { concentration } \\
\left(\mathrm{Ci} \mathrm{L}^{-1}\right)\end{array}$ & Radionuclide Group \\
\hline Am241 & $1.87 \mathrm{E}-02$ & $1.21 \mathrm{E}-05$ & $2.27 \mathrm{E}-07$ & & \\
\hline Pu238 & $4.61 \mathrm{E}-02$ & $8.90 \mathrm{E}-06$ & 4.10E-07 & $6.38 \mathrm{E}-07$ & $\mathrm{Pu}-238+\mathrm{Pu}-239+\mathrm{Am}-241$ \\
\hline Pu239 & $1.20 \mathrm{E}-04$ & $8.90 E-06$ & $1.07 \mathrm{E}-09$ & & \\
\hline Pu240 & $6.92 \mathrm{E}-03$ & $8.90 \mathrm{E}-06$ & $6.15 E-08$ & NA & NA \\
\hline Pu241 & $9.28 \mathrm{E}-01$ & $8.90 E-06$ & $8.25 \mathrm{E}-06$ & $8.25 \mathrm{E}-06$ & Pu-241 \\
\hline Pu242 & $4.38 \mathrm{E}-04$ & $8.90 \mathrm{E}-06$ & $3.90 \mathrm{E}-09$ & NA & NA \\
\hline
\end{tabular}


Table 4. Derived response levels for the milk pathway

\begin{tabular}{rccc}
\hline Radionuclide Group & $\begin{array}{l}\text { Radionuclide Group } \\
\text { concentration }\left(\mathrm{Ci} \mathrm{L}^{-1}\right) \\
\text { per unit deposition }\end{array}$ & DIL (Bq L $\left.{ }^{-1}\right)$ & $\begin{array}{l}\text { Deposition of mix corresponding } \\
\left.\text { to 1 DIL (Ci m }{ }^{-2}\right)\end{array}$ \\
\hline Pu-238 + Pu-239 + Am-241 & $6.38 \mathrm{E}-07$ & 2 & $8.47 \mathrm{E}-05$ \\
Pu-241 & $8.25 \mathrm{E}-06$ & 120 & $3.93 \mathrm{E}-04$ \\
\hline Reference: Source_H.xls, Worksheet "Dairy", columns N to Q
\end{tabular}

Table 4 presents depositions necessary to equal a DIL for the Pu-238 + Pu-239 + Am-241 group and the individual radionuclide $\mathrm{Pu}-241$. The activity of the depositions includes that from all radionuclides in the mix, even though the radionuclide(s) in a limiting group may only be a small fraction of the total activity released. The most limiting radionuclide group for the milk pathway is the $\mathrm{Pu}-238+\mathrm{Pu}-239+\mathrm{Am}-241$ group.

The processes outlined above may be repeated for other pathways. Table 5 lists total depositions of the radionuclide mix corresponding to $1 \mathrm{DIL}\left(\mathrm{Ci} \mathrm{m}^{-2}\right)$ of the $\mathrm{Pu}-238+$ $\mathrm{Pu}-239+\mathrm{Am}-241$ group and the individual radionuclide $\mathrm{Pu}-241$ for all pathways. Attachments B to $Q$ present spreadsheet calculations for each pathway.

Table 5. Derived response levels for all pathways

\begin{tabular}{lcc}
\hline Pathway & Deposition of mix $\left(\mathrm{Ci} \mathrm{m}\right.$ m $\left.^{-2}\right)$ corresponding to 1 DIL of nuclide group \\
\cline { 2 - 3 } Milk & Pu-238 + Pu-239+ Am-241 & Pu-241 \\
Egg contents & $8.47 \mathrm{E}-05$ & $3.93 \mathrm{E}-04$ \\
Beef & $1.67 \mathrm{E}-05$ & $2.11 \mathrm{E}-04$ \\
Veal & $5.96 \mathrm{E}-06$ & $4.66 \mathrm{E}-05$ \\
Sheep & $3.49 \mathrm{E}-07$ & $1.46 \mathrm{E}-06$ \\
Lamb & $4.08 \mathrm{E}-06$ & $2.27 \mathrm{E}-05$ \\
Pork & $1.77 \mathrm{E}-07$ & $8.12 \mathrm{E}-07$ \\
Poultry & $2.57 \mathrm{E}-06$ & $1.43 \mathrm{E}-05$ \\
Fish & $2.46 \mathrm{E}-06$ & $1.33 \mathrm{E}-05$ \\
Produce-direct deposition & $2.78 \mathrm{E}-08$ & $1.17 \mathrm{E}-07$ \\
Produce - root uptake & $2.91 \mathrm{E}-09$ & $1.22 \mathrm{E}-08$ \\
Produce-soil adhesion & $6.74 \mathrm{E}-03$ & $9.39 \mathrm{E}-02$ \\
Grain-direct deposition & $1.63 \mathrm{E}-04$ & $6.85 \mathrm{E}-04$ \\
Grain-root uptake & $2.91 \mathrm{E}-09$ & $1.22 \mathrm{E}-08$ \\
Grain-soil adhesion & $2.18 \mathrm{E}-02$ & $1.32 \mathrm{E}-01$ \\
Beverage & $6.78 \mathrm{E}-05$ & $2.85 \mathrm{E}-04$ \\
\hline
\end{tabular}

Reference: Source_H.xls, worksheets for each pathway 
In all cases, the $\mathrm{Pu}-238+\mathrm{Pu}-239+\mathrm{Am}-241$ group is more limiting than $\mathrm{Pu}-241$, reaching a DIL of the Pu-238 + Pu-239 + Am-241 group at a lower areal deposition than Pu-241. Since DILs are not additive between groups, the following discussion focuses on the $\mathrm{Pu}-238+\mathrm{Pu}-239+\mathrm{Am}-241$ group only.

Table 6 ranks the pathways by the deposition needed to reach a DIL of the Pu- $238+$ Pu-239+ Am-241 group.

Table 6 Food pathways ranked by DRL

\begin{tabular}{lc}
\hline Pathway & $\begin{array}{c}\text { Deposition of mix }\left(\mathrm{Ci} \mathrm{m}^{-2}\right) \text { corresponding to 1 DIL } \\
\text { of Pu-238 }\end{array}$ \\
\hline Produce-direct deposition & $2.91 \mathrm{E}-09$ \\
Grain-direct deposition & $2.91 \mathrm{E}-09$ \\
Fish & $2.78 \mathrm{E}-08$ \\
Lamb & $1.77 \mathrm{E}-07$ \\
Veal & $3.49 \mathrm{E}-07$ \\
Beverage & $8.33 \mathrm{E}-07$ \\
Poultry & $2.46 \mathrm{E}-06$ \\
Pork & $2.57 \mathrm{E}-06$ \\
Sheep & $4.08 \mathrm{E}-06$ \\
Beef & $5.96 \mathrm{E}-06$ \\
Egg contents & $1.67 \mathrm{E}-05$ \\
Grain-soil adhesion & $6.78 \mathrm{E}-05$ \\
Milk & $8.47 \mathrm{E}-05$ \\
Produce-soil adhesion & $1.63 \mathrm{E}-04$ \\
Produce - root uptake & $6.74 \mathrm{E}-03$ \\
Grain-root uptake & $2.18 \mathrm{E}-02$ \\
\hline
\end{tabular}

The direct deposition pathways reach the DIL first (i.e., they have the lowest DRLs). These pathways are important only for crops in the field at the time of passage of the residue cloud. The fish pathway is modeled for fish in stagnant ponds with no absorption of the radionuclides into the sediment. The ranking reflects the bioaccumulation factors for fish that are greater than unity. The meat pathways postulate ingestion of fodder contaminated by directly deposited radionuclides; fodder grown after the deposition and subsequently fed to animals would result in much lower concentrations in animal products.

\section{Determination of range and area of consequences}

The HOTSPOT computer code (Homann 1994) was selected to evaluate the consequences of particulate releases (WSRC-TR-98-00392). In the following calculations, a source term equal to the total activity $(35.1 \mathrm{Ci})$ is used, and the range and area affected for different meteorological parameters and DRLs found.

HOTSPOT Parameters used for the H-Area Coil \& Tube release are listed in Table 7. 
Table 7. HOTSPOT Parameters used for the H-Separations Coil \& Tube release (1-RD-2.35)

\begin{tabular}{lcc}
\hline Parameter & Average Meteorology & $95 \%$ Adverse Meteorology \\
\hline Release height & Ground & Ground \\
Wind speed & $2.5 \mathrm{~m} / \mathrm{s}$ & $1.7 \mathrm{~m} / \mathrm{s}$ \\
$\begin{array}{l}\text { Surface } \\
\text { roughness }\end{array}$ & $100 \mathrm{~cm}$ & $100 \mathrm{~cm}$ \\
$\begin{array}{l}\text { Stability class } \\
\begin{array}{l}\text { Deposition } \\
\text { velocity }\end{array}\end{array}$ & $\mathrm{C}$ & $\mathrm{E}$ \\
$\begin{array}{l}\text { Release duration } \\
\text { Inversion layer }\end{array}$ & $120 \mathrm{~min}$ & $0.1 / 1 / 10 \mathrm{~cm} / \mathrm{sec}$ \\
\hline
\end{tabular}

Meteorological data is consistent with Revision 1 of S-EHA-H-00004, Interim Emergency Preparedness Hazards Assessment for H Area Separations, Appendix B, Calculation 5, Sheet 1, which references Consequence Assessment for Emergency Preparedness Hazards Assessments. TP-95-002, Revision 3, January 21, 1997.

\section{Results}

Tables 8 and 9 summarize ingestion pathway consequences for average meteorology. If a contour (DRL) is not exceeded and/or the area affected is insignificant, "N/E" is entered for the value. Attachments $R$ through $T$ present the plots produced by the HOTSPOT runs for average meteorology. 
Table 8. Ranges until deposition less than DRL, average meteorology, H-Separations, $1-\mathrm{RD}-2.35$

\begin{tabular}{|l|c|c|c|c|}
\hline Pathway & $\begin{array}{c}\text { DRL } \\
\text { Ci m}\end{array}$ & $\begin{array}{c}0.1 \mathrm{~cm} \mathrm{~s}^{-1} \\
\mathrm{~km}\end{array}$ & $\begin{array}{c}1 \mathrm{~cm} \mathrm{~s}^{-1} \\
\mathrm{~km}\end{array}$ & $\begin{array}{c}10 \mathrm{~cm} \mathrm{~s}^{-1} \\
\mathrm{~km}\end{array}$ \\
\hline Produce-direct deposition & $2.91 \mathrm{E}-09$ & 40 & $>100$ & $>100$ \\
\hline Grain-direct deposition & $2.91 \mathrm{E}-09$ & 40 & $>100$ & $>100$ \\
\hline Fish & $2.78 \mathrm{E}-08$ & 1.5 & 40 & $>100$ \\
\hline Lamb & $1.77 \mathrm{E}-07$ & 0.6 & 2.0 & 15 \\
\hline Veal & $3.49 \mathrm{E}-07$ & 0.45 & 1.5 & 6.0 \\
\hline Beverage & $8.33 \mathrm{E}-07$ & 0.3 & 0.9 & 2.0 \\
\hline Poultry & $2.46 \mathrm{E}-06$ & 0.15 & 0.5 & 1.2 \\
\hline Pork & $2.57 \mathrm{E}-06$ & 0.15 & 0.5 & 1.2 \\
\hline Sheep & $4.08 \mathrm{E}-06$ & 0.10 & 0.4 & 0.9 \\
\hline Beef & $5.96 \mathrm{E}-06$ & 0.10 & 0.3 & 0.75 \\
\hline Egg contents & $1.67 \mathrm{E}-05$ & 0.06 & 0.2 & 0.5 \\
\hline Grain-soil adhesion & $6.78 \mathrm{E}-05$ & 0.03 & 0.1 & 0.25 \\
\hline Milk & $8.47 \mathrm{E}-05$ & 0.03 & 0.08 & 0.2 \\
\hline Produce-soil adhesion & $1.63 \mathrm{E}-04$ & 0.02 & 0.06 & 0.15 \\
\hline Produce - root uptake & $6.74 \mathrm{E}-03$ & N/E & 0.01 & 0.03 \\
\hline Grain-root uptake & $2.18 \mathrm{E}-02$ & N/E & N/E & 0.015 \\
\hline
\end{tabular}

Table 9. Areas above DRL, average meteorology, H-Separations, 1-RD-2.35

\begin{tabular}{|l|c|c|c|c|}
\hline Pathway & $\begin{array}{c}\mathrm{DRL} \\
\mathrm{Ci} \mathrm{m}-2\end{array}$ & $\begin{array}{c}0.1 \mathrm{~cm} \mathrm{~s}^{-1} \\
\mathrm{~km}^{2}\end{array}$ & $\begin{array}{c}1 \mathrm{~cm} \mathrm{~s}^{-1} \\
\mathrm{~km}^{2}\end{array}$ & $\begin{array}{c}10 \mathrm{~cm} \mathrm{~s}^{-1} \\
\mathrm{~km}^{2}\end{array}$ \\
\hline Produce-direct deposition & $2.91 \mathrm{E}-09$ & $1.0 \mathrm{E}+02$ & $>1.3 \mathrm{E}+03$ & $>1.6 \mathrm{E}+03$ \\
\hline Grain-direct deposition & $2.91 \mathrm{E}-09$ & $1.0 \mathrm{E}+02$ & $>1.3 \mathrm{E}+03$ & $>1.6 \mathrm{E}+03$ \\
\hline Fish & $2.78 \mathrm{E}-08$ & $5.8 \mathrm{E}-01$ & $8.6 \mathrm{E}+01$ & $>8.7 \mathrm{E}+02$ \\
\hline Lamb & $1.77 \mathrm{E}-07$ & $9.9 \mathrm{E}-02$ & $8.0 \mathrm{E}-01$ & $2.0 \mathrm{E}+01$ \\
\hline Veal & $3.49 \mathrm{E}-07$ & $5.0 \mathrm{E}-02$ & $4.8 \mathrm{E}-01$ & $4.7 \mathrm{E}+00$ \\
\hline Beverage & $8.33 \mathrm{E}-07$ & $2.1 \mathrm{E}-02$ & $2.0 \mathrm{E}-01$ & $9.8 \mathrm{E}-01$ \\
\hline Poultry & $2.46 \mathrm{E}-06$ & $6.7 \mathrm{E}-03$ & $6.7 \mathrm{E}-02$ & $3.5 \mathrm{E}-01$ \\
\hline Pork & $2.57 \mathrm{E}-06$ & $6.4 \mathrm{E}-03$ & $6.4 \mathrm{E}-02$ & $3.4 \mathrm{E}-01$ \\
\hline Sheep & $4.08 \mathrm{E}-06$ & $4.1 \mathrm{E}-03$ & $4.0 \mathrm{E}-02$ & $2.2 \mathrm{E}-01$ \\
\hline Beef & $5.96 \mathrm{E}-06$ & $2.6 \mathrm{E}-03$ & $2.8 \mathrm{E}-02$ & $1.5 \mathrm{E}-01$ \\
\hline Egg contents & $1.67 \mathrm{E}-05$ & N/E & $9.9 \mathrm{E}-03$ & $5.8 \mathrm{E}-02$ \\
\hline Grain-soil adhesion & $6.78 \mathrm{E}-05$ & N/E & N/E & $1.5 \mathrm{E}-02$ \\
\hline Milk & $8.47 \mathrm{E}-05$ & N/E & N/E & $1.3 \mathrm{E}-02$ \\
\hline Produce-soil adhesion & $1.63 \mathrm{E}-04$ & N/E & N/E & $6.7 \mathrm{E}-03$ \\
\hline Produce - root uptake & $6.74 \mathrm{E}-03$ & N/E & N/E & N/E \\
\hline Grain-root uptake & $2.18 \mathrm{E}-02$ & N/E & N/E & N/E \\
\hline
\end{tabular}


Tables 10 and 11 summarize ingestion pathway consequences for adverse meteorology. Plots from the HOTSPOT runs are included in Attachments $U$ through W.

Table 10. Ranges until deposition less than DRL, 95\% adverse meteorology, H-Separations, 1-RD-2.35

\begin{tabular}{|l|c|c|c|c|}
\hline Pathway & $\begin{array}{c}\text { DRL } \\
\text { Ci m}\end{array}$ & $\begin{array}{c}0.1 \mathrm{~cm} \mathrm{~s}^{-1} \\
\mathrm{~km}\end{array}$ & $\begin{array}{c}1 \mathrm{~cm} \mathrm{~s}^{-1} \\
\mathrm{~km}^{-1}\end{array}$ & $\begin{array}{c}10 \mathrm{~cm} \mathrm{~s}^{-1} \\
\mathrm{~km}\end{array}$ \\
\hline Produce-direct deposition & $2.91 \mathrm{E}-09$ & $>100$ & $>100$ & $>100$ \\
\hline Grain-direct deposition & $2.91 \mathrm{E}-09$ & $>100$ & $>100$ & $>100$ \\
\hline Fish & $2.78 \mathrm{E}-08$ & 25 & $>100$ & 32 \\
\hline Lamb & $1.77 \mathrm{E}-07$ & 3 & 30 & 7 \\
\hline Veal & $3.49 \mathrm{E}-07$ & 1.8 & 11 & 4 \\
\hline Beverage & $8.33 \mathrm{E}-07$ & 1.0 & 4 & 2.5 \\
\hline Poultry & $2.46 \mathrm{E}-06$ & 0.5 & 2 & 1.5 \\
\hline Pork & $2.57 \mathrm{E}-06$ & 0.5 & 2 & 1.5 \\
\hline Sheep & $4.08 \mathrm{E}-06$ & 0.4 & 1.4 & 1.1 \\
\hline Beef & $5.96 \mathrm{E}-06$ & 0.3 & 1.0 & 0.9 \\
\hline Egg contents & $1.67 \mathrm{E}-05$ & 0.2 & 0.6 & 0.6 \\
\hline Grain-soil adhesion & $6.78 \mathrm{E}-05$ & 0.09 & 0.3 & 0.3 \\
\hline Milk & $8.47 \mathrm{E}-05$ & 0.08 & 0.2 & 0.28 \\
\hline Produce-soil adhesion & $1.63 \mathrm{E}-04$ & 0.06 & 0.16 & 0.25 \\
\hline Produce - root uptake & $6.74 \mathrm{E}-03$ & N/E & 0.025 & 0.05 \\
\hline Grain-root uptake & $2.18 \mathrm{E}-02$ & N/E & 0.015 & 0.035 \\
\hline
\end{tabular}

Table 11. Areas above DRL, 95\% adverse meteorology, H-Separations, 1-RD-2.35

\begin{tabular}{|l|c|c|c|c|}
\hline Pathway & $\begin{array}{c}\text { DRL } \\
\mathrm{Ci} \mathrm{m}\end{array}$ & $\begin{array}{c}0.1 \mathrm{~cm} \mathrm{~s}^{-1} \\
\mathrm{~km}^{2}\end{array}$ & $\begin{array}{c}1 \mathrm{~cm} \mathrm{~s}^{-1} \\
\mathrm{~km}^{2}\end{array}$ & $\begin{array}{c}10 \mathrm{~cm} \mathrm{~s}^{-1} \\
\mathrm{~km}^{2}\end{array}$ \\
\hline Produce-direct deposition & $2.91 \mathrm{E}-09$ & $>6.0 \mathrm{E}+02$ & $>8.6 \mathrm{E}+02$ & $>5.7 \mathrm{E}+02$ \\
\hline Grain-direct deposition & $2.91 \mathrm{E}-09$ & $>6.0 \mathrm{E}+02$ & $>8.6 \mathrm{E}+02$ & $>5.7 \mathrm{E}+02$ \\
\hline Fish & $2.78 \mathrm{E}-08$ & $2.2 \mathrm{E}+01$ & $>5.3 \mathrm{E}+02$ & $3.9 \mathrm{E}+01$ \\
\hline Lamb & $1.77 \mathrm{E}-07$ & $7.3 \mathrm{E}-01$ & $2.6 \mathrm{E}+01$ & $3.3 \mathrm{E}+00$ \\
\hline Veal & $3.49 \mathrm{E}-07$ & $3.2 \mathrm{E}-01$ & $5.1 \mathrm{E}+00$ & $1.8 \mathrm{E}+00$ \\
\hline Beverage & $8.33 \mathrm{E}-07$ & $1.1 \mathrm{E}-01$ & $1.4 \mathrm{E}+00$ & $7.5 \mathrm{E}-01$ \\
\hline Poultry & $2.46 \mathrm{E}-06$ & $3.2 \mathrm{E}-02$ & $3.5 \mathrm{E}-01$ & $2.7 \mathrm{E}-01$ \\
\hline Pork & $2.57 \mathrm{E}-06$ & $3.1 \mathrm{E}-02$ & $3.4 \mathrm{E}-01$ & $2.6 \mathrm{E}-01$ \\
\hline Sheep & $4.08 \mathrm{E}-06$ & $1.9 \mathrm{E}-02$ & $2.0 \mathrm{E}-01$ & $1.7 \mathrm{E}-01$ \\
\hline Beef & $5.96 \mathrm{E}-06$ & $1.2 \mathrm{E}-02$ & $1.3 \mathrm{E}-01$ & $1.3 \mathrm{E}-01$ \\
\hline Egg contents & $1.67 \mathrm{E}-05$ & $4.1 \mathrm{E}-03$ & $4.0 \mathrm{E}-02$ & $5.2 \mathrm{E}-02$ \\
\hline Grain-soil adhesion & $6.78 \mathrm{E}-05$ & N/E & $8.9 \mathrm{E}-03$ & $1.6 \mathrm{E}-02$ \\
\hline Milk & $8.47 \mathrm{E}-05$ & N/E & $7.1 \mathrm{E}-03$ & $1.4 \mathrm{E}-02$ \\
\hline Produce-soil adhesion & $1.63 \mathrm{E}-04$ & N/E & $3.7 \mathrm{E}-03$ & $8.0 \mathrm{E}-03$ \\
\hline Produce - root uptake & $6.74 \mathrm{E}-03$ & N/E & N/E & N/E \\
\hline Grain-root uptake & $2.18 \mathrm{E}-02$ & N/E & N/E & N/E \\
\hline
\end{tabular}




\section{Conclusion}

Tables 8 to 11 present ingestion pathway consequences from H-Separations accident scenario 1-RD-2.35, Coil and Tube Failure. The Pu-238 + Pu-239 + Am-241 group is the most limiting for all pathways. The most significant pathways are those involving ingestion of freshly deposited radionuclides on produce and grain. Contamination of produce and grain exceeds FDA guidelines out to at least $40 \mathrm{~km}$ in all cases, and out to greater than $100 \mathrm{~km}$ in most cases. Additionally, under some conditions, it may be possible for fish and lamb foodstuffs to exceed FDA guidelines offsite. Sampling and analysis of these pathways would be recommended. Ranges for all other pathways are less than the distance to the site boundary (approximately $11 \mathrm{~km}$ ), so protective actions for these other pathways would not be recommended for offsite locations.

\section{References}

Burns, R. D. and Clifton, L. L. Interim Emergency Preparedness Hazards Assessment for H Area Separations, Aiken, SC: Westinghouse Savannah River Company; S-EHA-H-00004, Rev. 1; June 1998.

Eisele, D. P. Risks of $F \& H$ Cooling Tower Airborne Release (U), Aiken, SC: Westinghouse Savannah River Company; S-CLC-G-00004, Rev. 0; May 1994.

Food and Drug Administration. Accidental radioactive contamination of human foods and animal feeds: recommendations for state and local agencies. Rockville, MD: FDA; August 13, 1998.

Hadlock, D. J., Consequence Assessment for Emergency Preparedness Hazards Assessments, Aiken, SC: Westinghouse Savannah River Company; TP-95-002, Rev. 3, 1/20/97.

Homann, Steven G., HOTSPOT Health Physics Codes for the PC, UCRL-MA-106315, Lawrence Livermore National Laboratory, University of California, Livermore, CA, March 1994.

Tharakan, B.K. Derived Intervention Levels for Tritium Based on Food and Drug Administration Methodology Using ICRP 56 Dose Coefficients (U), Aiken, SC; Westinghouse Savannah River Company; WSRC-TR-99-00064, Rev. 0; February 1999.

Thompson, J. M. Ingestion Pathway Transfer Factors for Plutonium and Americium (U), Aiken, SC; Westinghouse Savannah River Company; WSRC-TR-99-00005, Rev. 0; January 1999.

Thompson, J. M. Selection of Models for Ingestion Pathway and Relocation (U), Aiken, SC; Westinghouse Savannah River Company; WSRC-TR-98-00392, Rev. 2; February, 1999. 


\section{Attachments}

Attachment A

Source term and areal concentrations spreadsheet calculations

Attachment B

Contamination of dairy products spreadsheet calculations

Attachment C

Contamination of egg contents spreadsheet calculations

Attachment D

Contamination of beef spreadsheet calculations

Attachment E

Contamination of veal spreadsheet calculations

Attachment F

Contamination of sheep spreadsheet calculations

Attachment G

Contamination of lamb spreadsheet calculations

Attachment $\mathrm{H}$

Contamination of pork spreadsheet calculations

Attachment I

Contamination of poultry spreadsheet calculations

Attachment J

Contamination of fish spreadsheet calculations

Attachment $\mathrm{K}$

External contamination of produce from direct deposition spreadsheet calculations

Attachment L

Internal contamination of produce from root uptake spreadsheet calculations

Attachment M

External contamination of leafy vegetables during harvest spreadsheet calculations

Attachment $\mathrm{N}$

External contamination of grain spreadsheet calculations 
Attachment $\mathrm{O}$

Internal contamination of grain by root uptake spreadsheet calculations

Attachment $P$

External contamination of grain during harvest spreadsheet calculations

Attachment Q

Contamination of beverages spreadsheet calculations

Attachment $\mathrm{R}$

HOTSPOT results - average meteorology, $0.1 \mathrm{~cm} \mathrm{~s}^{-1}$

Attachment S

HOTSPOT results - average meteorology, $1.0 \mathrm{~cm} \mathrm{~s}^{-1}$

Attachment T

HOTSPOT results - average meteorology, $10 \mathrm{~cm} \mathrm{~s}^{-1}$

Attachment U

HOTSPOT results - adverse meteorology, $0.1 \mathrm{~cm} \mathrm{~s}^{-1}$

Attachment V

HOTSPOT results - adverse meteorology, $1.0 \mathrm{~cm} \mathrm{~s}^{-1}$

Attachment W

HOTSPOT results - adverse meteorology, $10 \mathrm{~cm} \mathrm{~s}^{-1}$ 
Attachment A

Source term and areal concentrations spreadsheet calculations 


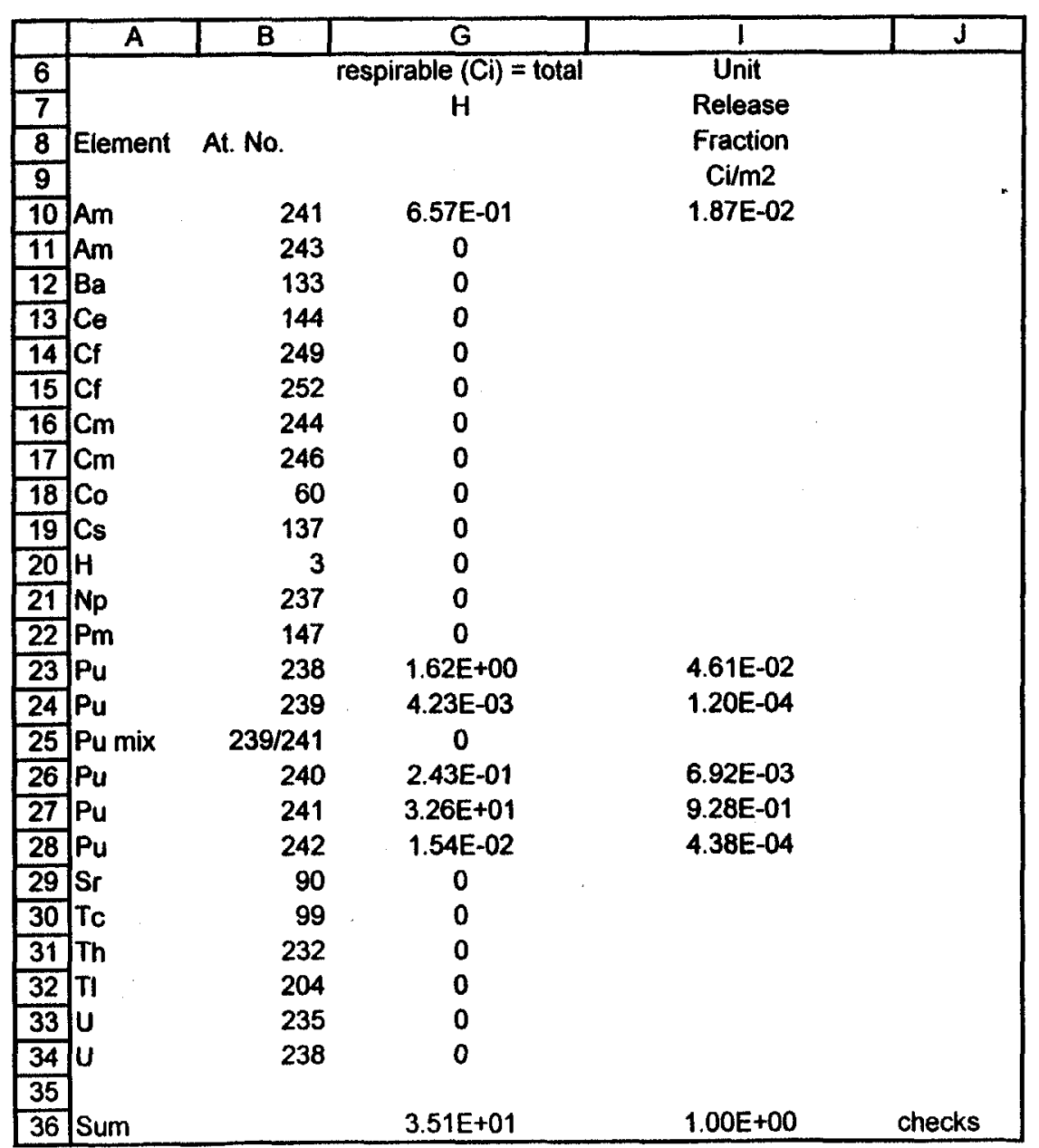




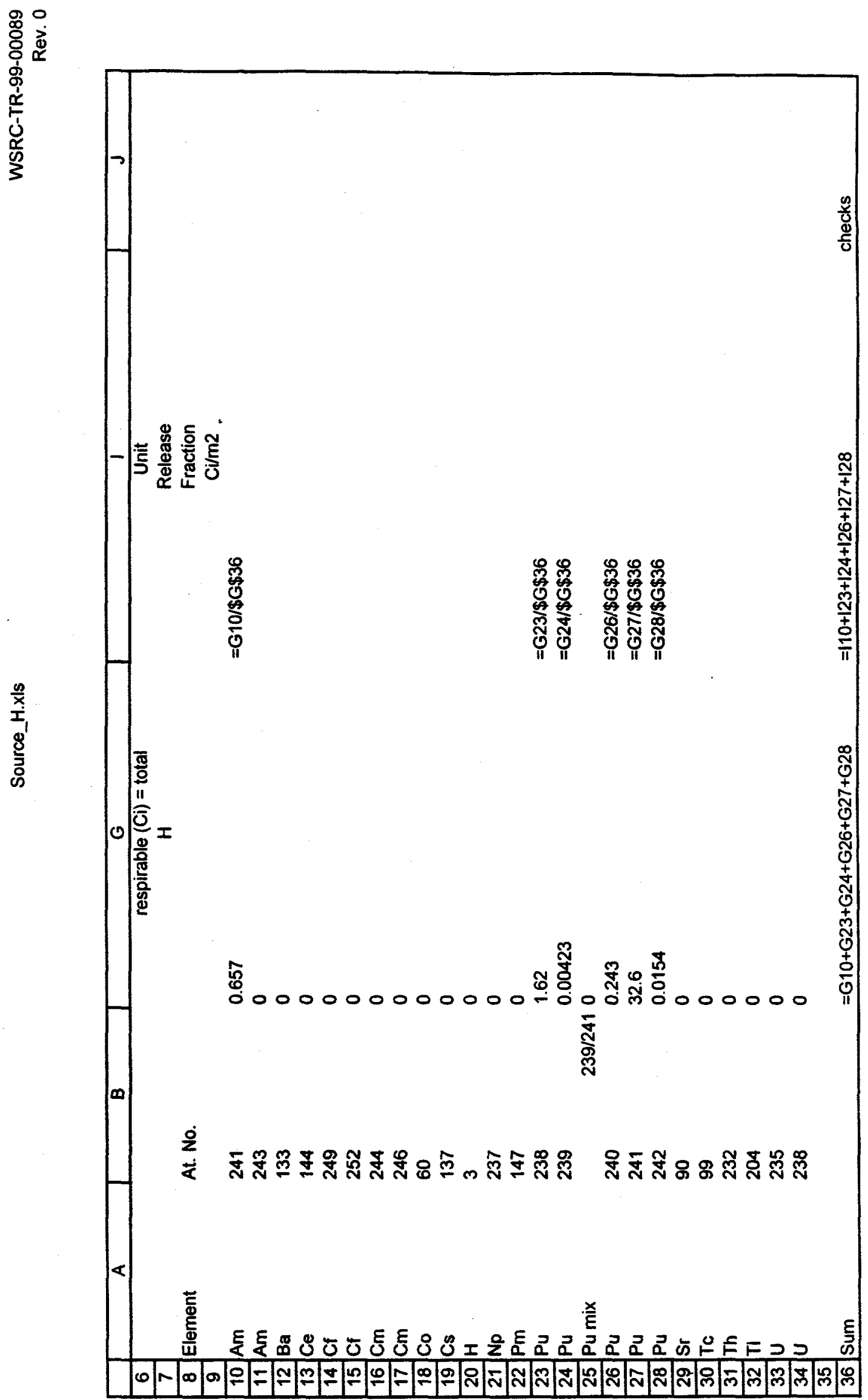

खे 


\section{Attachment B}

Contamination of dairy products spreadsheet calculations 


\begin{tabular}{|c|c|c|c|c|c|c|c|c|}
\hline & A & B & $\mathbf{L}$ & M & $\mathbf{N}$ & $\mathbf{0}$ & $\mathbf{P}$ & $\mathbf{Q}$ \\
\hline 2 & \multicolumn{8}{|c|}{ Dairy } \\
\hline 3 & \multirow{2}{*}{\multicolumn{5}{|c|}{ Consumption of direct deposition on pasture }} & Group & \multicolumn{2}{|c|}{ Group DIL Total } \\
\hline 4 & & & & & & sum & & Dep for \\
\hline 5 & & & & DEP & & & $\mathrm{Bq} / \mathrm{L}$ & DIL \\
\hline 6 & & & Tmilk & Unit & $T \times$ DEP & & & $\mathrm{Ci} / \mathrm{m} 2$ \\
\hline 7 & & & & Release & $\mathrm{Ci} / \mathrm{L}$ & Ci/L & & 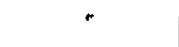 \\
\hline 8 & Element & At. No. & $\mathrm{m} 2 / \mathrm{L}$ & Fraction & & & & \\
\hline 9 & & & & $\mathrm{Ci} / \mathrm{m} 2$ & & & & \\
\hline 10 & Am & 241 & 1.21E-05 & 1.87E-02 & $2.27 \mathrm{E}-07$ & & & \\
\hline 11 & Am & 243 & 1.21E-05 & & & & & \\
\hline 12 & $\mathrm{Ba}$ & 133 & $3.88 E-03$ & & & & & \\
\hline 13 & $\mathrm{Ce}$ & 144 & 2.43E-04 & & & & & \\
\hline 14 & $\mathrm{Cf}$ & 249 & 1.62E-05 & & & & & \\
\hline 15 & $\mathrm{Cf}$ & 252 & 1.62E-05 & & Am241 & & & \\
\hline 16 & $\mathrm{Cm}$ & 244 & 1.62E-05 & & Pu238 & $6.38 \mathrm{E}-07$ & 2 & 8.47E-05 \\
\hline 17 & $\mathrm{Cm}$ & 246 & 1.62E-05 & & Pu239 & & & \\
\hline 18 & Co & 60 & 2.43E-03 & & & & & \\
\hline 19 & Cs & 137 & 6.39E-02 & & & & & \\
\hline 20 & $H$ & 3 & 1.21E-01 & & & & & \\
\hline 21 & $\mathrm{~Np}$ & 237 & 4.04E-05 & & & & & \\
\hline 22 & Pm & 147 & 4.85E-04 & & & & & \\
\hline 23 & Pu & 238 & $8.90 E-06$ & 4.61E-02 & 4.10E-07 & & & \\
\hline 24 & $\mathrm{Pu}$ & 239 & 8.90E-06 & $1.20 \mathrm{E}-04$ & 1.07E-09 & & & \\
\hline 25 & $\mathrm{Pu}$ & $39 / 241$ & 8.90E-06 & & & & & \\
\hline 26 & $\mathrm{Pu}$ & 240 & 8.90E-06 & 6.92E-03 & 6.15E-08 & & & \\
\hline 27 & $\mathrm{Pu}$ & 241 & 8.90E-06 & $9.28 E-01$ & 8.25E-06 & 8.25E-06 & 120 & $3.93 E-04$ \\
\hline 28 & $\mathrm{Pu}$ & 242 & 8.90E-06 & 4.38E-04 & $3.90 \mathrm{E}-09$ & & & \\
\hline 29 & $\mathrm{Sr}$ & 90 & 2.26E-02 & & & & & \\
\hline 30 & Tc & 99 & 1.86E-04 & & & & & \\
\hline 31 & Th & 232 & 4.04E-05 & & & & & \\
\hline 32 & $T \mid$ & 204 & 2.43E-02 & & & & & \\
\hline 33 & $\mathrm{u}$ & 235 & $3.24 E-03$ & & & & & \\
\hline 34 & $u$ & 238 & 3.24E-03 & & & & & \\
\hline
\end{tabular}




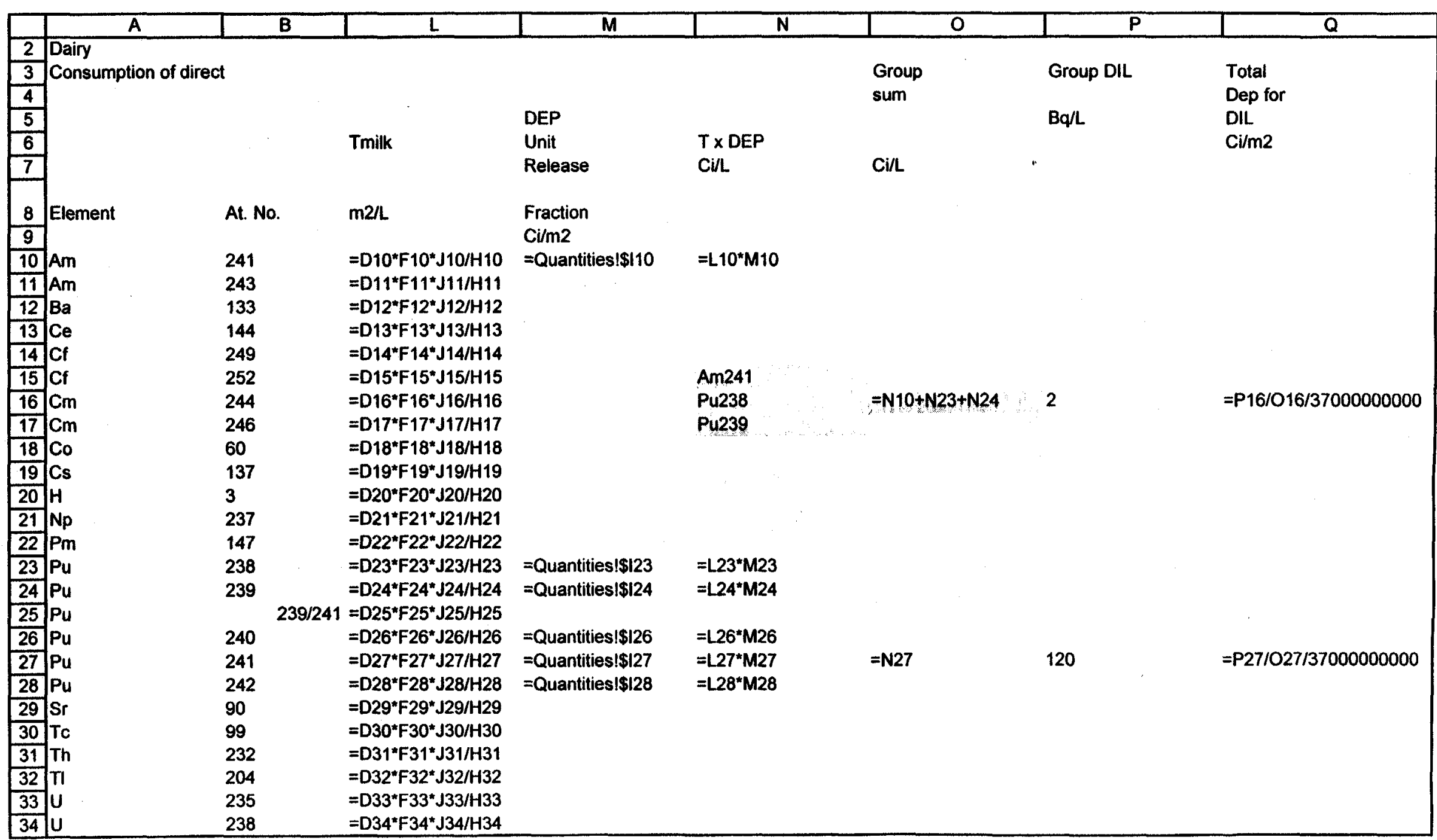




\section{Attachment C}

Contamination of egg contents spreadsheet calculations 


\begin{tabular}{|c|c|c|c|c|c|c|c|c|}
\hline & $\bar{A}$ & B & $\mathrm{L}$ & $\bar{M}$ & $\overline{\mathbf{N}}$ & 0 & $P$ & $\mathbf{Q}$ \\
\hline 2 & \multicolumn{8}{|c|}{ Egg contents } \\
\hline 3 & & & \multirow{5}{*}{ Teggs } & \multirow{5}{*}{$\begin{array}{l}\text { Unit } \\
\text { Release }\end{array}$} & \multirow{4}{*}{$T \times D E P$} & \multirow{4}{*}{$\begin{array}{l}\text { Group } \\
\text { sum }\end{array}$} & Group DIL & Total \\
\hline 4 & & & & & & & & Dep for \\
\hline 5 & & & & & & & $\mathrm{Ci} / \mathrm{kg}$ & DIL \\
\hline 6 & & & & & & & & $\mathrm{Ci} / \mathrm{m} 2$ \\
\hline 7 & & & & & Ci/kg & $\mathrm{Ci} / \mathrm{kg}$ & & \\
\hline 8 & Elemen & At. No. & $\mathrm{m} 2 / \mathrm{kg}$ & Fraction & & & & \\
\hline 9 & & & & $\mathrm{Ci} / \mathrm{m} 2$ & & & & \\
\hline 10 & Am & 241 & 1.33E-04 & 1.87E-02 & $2.48 E-06$ & & & \\
\hline 11 & Am & 243 & 1.33E-04 & & & & & \\
\hline 12 & $\mathrm{Ba}$ & 133 & 2.98E-02 & & & & & \\
\hline 13 & $\mathrm{Ce}$ & 144 & 2.98E-06 & & & & & \\
\hline 14 & $\mathrm{Cf}$ & 249 & & & & & & \\
\hline 15 & Cf & 252 & & & Am241 & & & \\
\hline 16 & $\mathrm{~cm}$ & 244 & & & Pu238 & $3.24 \mathrm{E}-0.6$ & 2 & 1.67E-05 \\
\hline 17 & $\mathrm{Cm}$ & 246 & & & Pu239 & & & \\
\hline 18 & Co & 60 & 3.31E-03 & & & & & \\
\hline 19 & Cs & 137 & 1.33E-02 & & & & & \\
\hline 20 & $\mathrm{H}$ & 3 & & & & & & \\
\hline 21 & Np & 237 & & & & & & \\
\hline 22 & $\mathrm{Pm}$ & 147 & 6.63E-04 & & & & & \\
\hline 23 & $\mathrm{Pu}$ & 238 & 1.66E-05 & 4.61E-02 & $7.64 \mathrm{E}-07$ & & & \\
\hline 24 & $\mathrm{Pu}$ & 239 & 1.66E-05 & $1.20 E-04$ & 1.99E-09 & & & \\
\hline 25 & $\mathrm{Pu}$ & $39 / 241$ & 1.66E-05 & & & & & \\
\hline 26 & $\mathrm{Pu}$ & 240 & $1.66 \mathrm{E}-05$ & $6.92 E-03$ & $1.15 \mathrm{E}-07$ & & & \\
\hline 27 & $\mathrm{Pu}$ & 241 & 1.66E-05 & $9.28 E-01$ & $1.54 \mathrm{E}-0.5$ & $1.54 E-05$ & 120 & 2.11E-04 \\
\hline 28 & $\mathrm{Pu}$ & 242 & 1.66E-05 & 4.38E-04 & $7.26 \mathrm{E}-09$ & & & \\
\hline 29 & $\mathrm{sr}$ & 90 & $6.63 E-03$ & & & & & \\
\hline 30 & Tc & 99 & $9.94 E-02$ & & & & & \\
\hline 31 & Th & 232 & & & & & & \\
\hline 32 & TI & 204 & & & & & & \\
\hline 33 & $u$ & 235 & $3.31 E-02$ & & & & & \\
\hline 34 & $u$ & 238 & 3.31E-02 & & & & & \\
\hline
\end{tabular}




\begin{tabular}{|c|c|c|c|c|c|c|c|c|}
\hline & $\bar{A}$ & $\bar{B}$ & $L$ & $\mathbf{M}$ & $\mathbf{N}$ & $\mathbf{0}$ & $\mathbf{P}$ & $\mathbf{Q}$ \\
\hline 2 & Egg contents & & & & & & & \\
\hline 3 & & & & & & Group & Group DIL & Total \\
\hline 4 & & & & & & sum & & Dep for \\
\hline 5 & & & & & & & $\mathrm{Ci} / \mathrm{kg}$ & DIL \\
\hline 6 & & & Teggs & Unit & $T \times D E P$ & & & $\mathrm{Ci} / \mathrm{m} 2$ \\
\hline 7 & & & & Release & Ci/kg & $\mathrm{Ci} / \mathrm{kg}$ & " & \\
\hline 8 & Element & At. No. & $\mathrm{m} 2 / \mathrm{kg}$ & Fraction & & & & \\
\hline 9 & & & & $\mathrm{Ci} / \mathrm{m} 2$ & & & & \\
\hline 10 & Am & 241 & $=\mathrm{D} 10^{*} \mathrm{~F} 10^{*} \mathrm{~J} 10 / \mathrm{H} 10$ & =Quantities!\$110 & $=L 10^{*} M 10$ & & & \\
\hline 11 & Am & 243 & $=\mathrm{D} 11^{*} \mathrm{~F} 11^{*} \mathrm{~J} 11 / \mathrm{H} 11$ & & & & & \\
\hline 12 & $\mathrm{Ba}$ & 133 & $=D 12^{*} F 12^{*} \mathrm{~J} 12 / \mathrm{H} 12$ & & & & & \\
\hline 13 & $\mathrm{Ce}$ & 144 & $=D 13^{*} F 13^{*} \mathrm{~J} 13 / \mathrm{H} 13$ & & & & & \\
\hline 14 & Cf & 249 & & & & & & \\
\hline 15 & Cf & 252 & & & Am241 & & & \\
\hline 16 & $\mathrm{Cm}$ & 244 & & & Pu238 & $=N 10+N 23+N 24$ & 2 & $=\mathrm{P} 16 / 016 / 37000000000$ \\
\hline 17 & $\mathrm{Cm}$ & 246 & & & Pu239 & & & \\
\hline 18 & Co & 60 & $=D 18^{*} F 18^{*} J 18 / H 18$ & & & & & \\
\hline 19 & Cs & 137 & $=D 19^{*} F 19^{*} \mathrm{~J} 19 / \mathrm{H} 19$ & & & & & \\
\hline 20 & H & 3 & & & & & & \\
\hline 21 & Np & 237 & & & & & & \\
\hline 22 & $\mathrm{Pm}$ & 147 & $=\mathrm{D} 22^{\star} \mathrm{F} 22^{*} \mathrm{~J} 22 / \mathrm{H} 22$ & & & & & \\
\hline 23 & $\mathrm{Pu}$ & 238 & $=\mathrm{D} 23^{*} \mathrm{~F} 23^{*} \mathrm{~J} 23 / \mathrm{H} 23$ & $=$ Quantities $1 \$ 123$ & $=L 23^{*} M 23$ & & & \\
\hline 24 & $\mathrm{Pu}$ & 239 & $=D 24^{*} F 24^{*} \mathrm{~J} 24 / \mathrm{H} 24$ & $=$ Quantities $1 \$ 124$ & $=L 24^{*} M 24$ & & & \\
\hline 25 & $\mathrm{Pu}$ & & $=\mathrm{D} 25^{\star} \mathrm{F} 25^{\star} \mathrm{J} 25 / \mathrm{H} 25$ & & & & & \\
\hline 26 & $\mathrm{Pu}$ & 240 & $=\mathrm{D} 26^{*} \mathrm{~F} 26^{*} \mathrm{~J} 26 / \mathrm{H} 26$ & $=$ Quantities!\$126 & $=\mathrm{L} 26^{*} \mathrm{M} 26$ & & & \\
\hline 27 & $\mathrm{Pu}$ & 241 & $=\mathrm{D} 27^{*} \mathrm{~F} 27^{*} \mathrm{~J} 27 / \mathrm{H} 27$ & $=$ Quantities $1 \$ 127$ & $=\mathrm{L} 27^{*} \mathrm{M} 27$ & $=\mathrm{N} 27$ & 120 & $=\mathrm{P} 27 / \mathrm{O} 27 / 37000000000$ \\
\hline 28 & $\mathrm{Pu}$ & 242 & $=D 28^{*} F 28^{*} \mathrm{~J} 28 / \mathrm{H} 28$ & $=$ Quantities $\$ \$ 128$ & $=\mathrm{L} 28^{*} \mathrm{M} 28$ & & & \\
\hline 29 & $\mathrm{Sr}$ & 90 & $=D 29^{*} F 29^{*} \mathrm{~J} 29 / \mathrm{H} 29$ & & & & & \\
\hline 30 & Tc & 99 & $=\mathrm{D} 30^{*} \mathrm{~F} 30^{*} \mathrm{~J} 30 / \mathrm{H} 30$ & & & & & \\
\hline 31 & Th & 232 & & & & & & \\
\hline 32 & $\mathrm{TI}$ & 204 & & & & & & \\
\hline 33 & u & 235 & $=D 33^{*} F 33^{*} J 33 / H 33$ & & & & & \\
\hline 34 & $\mathrm{u}$ & 238 & $=D 34^{*} F 34^{*} \mathrm{~J} 34 / \mathrm{H} 34$ & & & & & \\
\hline
\end{tabular}




\section{Attachment D}

Contamination of beef spreadsheet calculations 


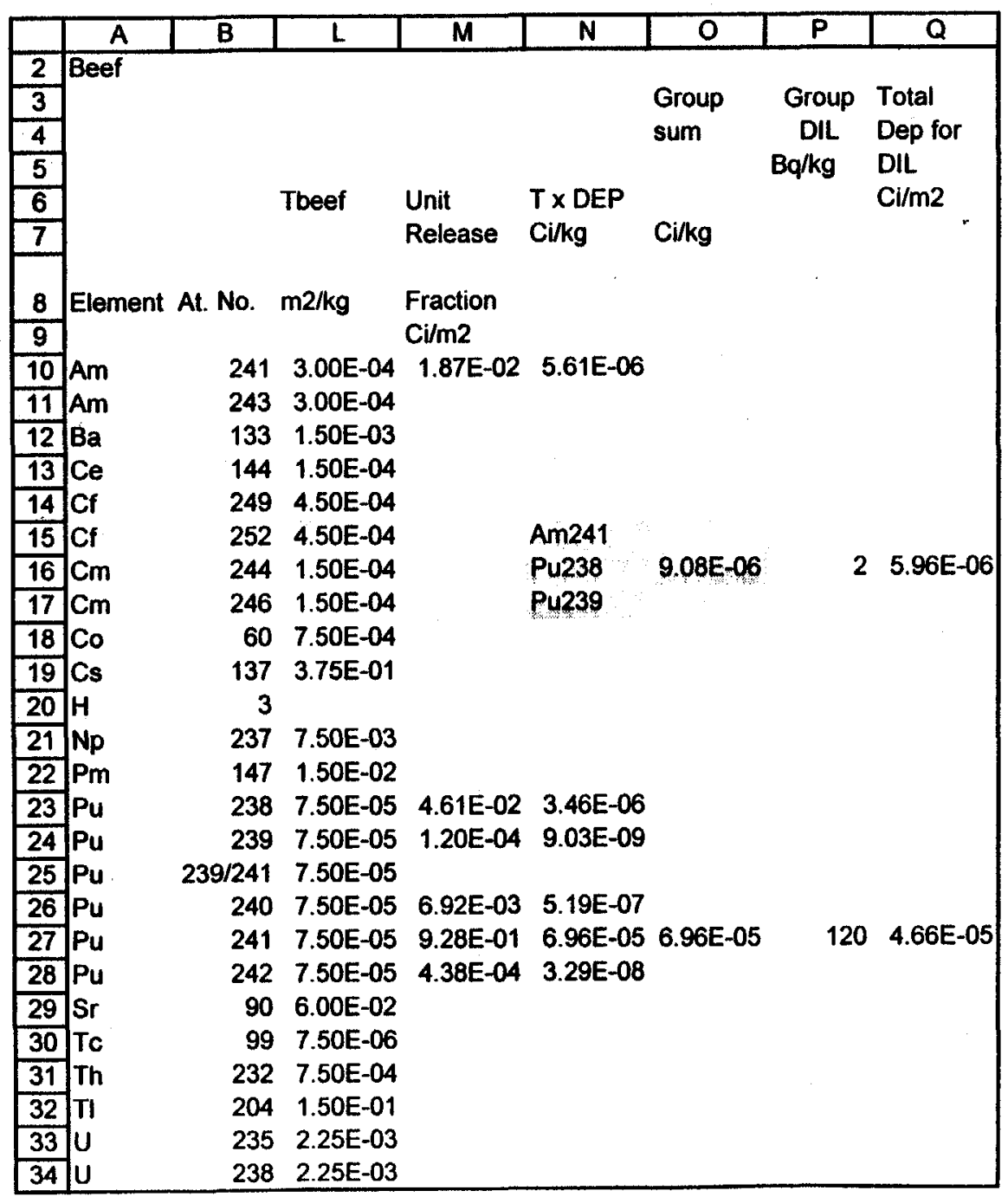









\section{Attachment E}

Contamination of veal spreadsheet calculations 
Source_H.xls

\begin{tabular}{|c|c|c|c|c|c|c|c|c|}
\hline & $A$ & B & $L$ & $\bar{M}$ & $\mathbf{N}$ & 0 & $\mathbf{P}$ & $\mathbf{Q}$ \\
\hline 2 & Veal & & & & & & & 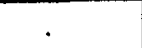 \\
\hline 3 & & & & & & Group & $\begin{array}{l}\text { Group } \\
\text { DIL }\end{array}$ & Total \\
\hline 4 & & & & & & sum & & Dep for \\
\hline 5 & & & & & & & $\mathrm{~Bq} / \mathrm{kg}$ & DIL \\
\hline 6 & & & Tveal & Unit & $T \times D E P$ & & & \\
\hline 7 & & & & Release & Ci/kg & Ci/kg & & \\
\hline 8 & Element & At. No. & $\mathrm{m} 2 / \mathrm{kg}$ & Fraction & & & & \\
\hline 9 & & & & $\mathrm{Ci} / \mathrm{m} 2$ & & & & \\
\hline 10 & Am & 241 & $2.39 E-03$ & 1.87E-02 & 4.47E-05 & & & \\
\hline 11 & Am & 243 & 2.39E-03 & & & & & \\
\hline 12 & $\mathrm{Ba}$ & 133 & & & & & & \\
\hline 13 & $\mathrm{Ce}$ & 144 & & & & & & \\
\hline 14 & Cf & 249 & & & & & & \\
\hline 15 & Cf & 252 & & & Am241 & & & \\
\hline 16 & $\mathrm{Cm}$ & 244 & & & Pu238 & $1.55 \mathrm{E}-04$ & & $3.49 E-07$ \\
\hline 17 & $\mathrm{Cm}$ & 246 & & & Pu239 & & & \\
\hline 18 & Co & 60 & & & & & & \\
\hline 19 & Cs & 137 & $4.78 E-01$ & & & & & \\
\hline 20 & $H$ & 3 & & & & & & \\
\hline 21 & $\mathrm{~Np}$ & 237 & & & & & & \\
\hline 22 & $\mathrm{Pm}$ & 147 & & & & & & \\
\hline 23 & $\mathrm{Pu}$ & 238 & 2.39E-03 & 4.61E-02 & 1.10E-04 & & & \\
\hline 24 & $\mathrm{Pu}$ & 239 & 2.39E-03 & 1.20E-04 & 2.88E-07 & & & \\
\hline 25 & $\mathrm{Pu}$ & $39 / 241$ & 2.39E-03 & & & & & \\
\hline 26 & $\mathrm{Pu}$ & 240 & 2.39E-03 & $6.92 E-03$ & 1.65E-05 & & & \\
\hline 27 & $\mathrm{Pu}$ & 241 & 2.39E-03 & $9.28 E-01$ & 2.22E-03 & 2.22E-03 & 120 & $1.46 \mathrm{E}-06$ \\
\hline 28 & $\mathrm{Pu}$ & 242 & 2.39E-03 & 4.38E-04 & 1.05E-06 & & & \\
\hline 29 & $\mathrm{Sr}$ & 90 & 2.39E-01 & & & & & \\
\hline 30 & TC & 99 & & & & & & \\
\hline 31 & Th & 232 & & & & & & \\
\hline 32 & $\mathrm{TI}$ & 204 & & & & & & \\
\hline 33 & $u$ & 235 & & & & & & \\
\hline 34 & u & 238 & & & & & & \\
\hline
\end{tabular}




\begin{tabular}{|c|c|c|c|c|c|c|c|c|}
\hline & $\bar{A}$ & $\bar{B}$ & $L$ & $\bar{M}$ & $\mathbf{N}$ & 0 & $P$ & $\mathbf{Q}$ \\
\hline 2 & \multicolumn{8}{|l|}{ Veal } \\
\hline 3 & & & & & & Group & Group DIL & Total \\
\hline$\frac{4}{5}$ & & & & & & sum & & Dep for \\
\hline$\frac{5}{6}$ & & & Treal & Unit & $T \times D E P$ & & $\mathbf{B q} / \mathbf{k g}$ & DIL \\
\hline 7 & & & & Release & Ci/kg & $\mathrm{Ci} / \mathrm{kg}$ & & \\
\hline 8 & Element & At. No. & $\mathrm{m} 2 / \mathrm{kg}$ & Fraction & & & & \\
\hline 9 & & & & $\mathrm{Ci} / \mathrm{m} 2$ & & & & \\
\hline 10 & Am & 241 & $=\mathrm{D} 10^{*} \mathrm{~F} 10^{*} \mathrm{~J} 10 / \mathrm{H} 10$ & $=$ Quantities $! \$ 10$ & $=L 10^{*} M 10$ & & & \\
\hline 11 & Am & 243 & $=\mathrm{D} 11^{*} \mathrm{~F} 11^{*} \mathrm{~J} 11 / \mathrm{H} 11$ & & & & & \\
\hline 12 & $\mathrm{Ba}$ & 133 & & & & & & \\
\hline 13 & $\mathrm{Ce}$ & 144 & & & & & & \\
\hline 14 & Cf & 249 & & & & & & \\
\hline 15 & $\mathrm{Cf}$ & 252 & & & Am241 & & & \\
\hline 16 & $\mathrm{Cm}$ & 244 & & & Pu238 & $=N 10+N 23+N 24$ & 2 & $=\mathrm{P} 16 / 016 / 37000000000$ \\
\hline 17 & $\mathrm{Cm}$ & 246 & & & Pu239 & & & \\
\hline 18 & Co & 60 & & & & & & \\
\hline 19 & Cs & 137 & $=D 19^{*} F 19^{*} J 19 / H 19$ & & & & & \\
\hline 20 & $H$ & 3 & & & & & & - \\
\hline 21 & $\mathrm{~Np}$ & 237 & & & & & & \\
\hline 22 & $\mathrm{Pm}$ & 147 & & & & & & \\
\hline 23 & $\mathrm{Pu}$ & 238 & $=\mathrm{D} 23^{*} \mathrm{~F} 23^{*} \mathrm{~J} 23 / \mathrm{H} 23$ & $=$ Quantities $1 \$ 123$ & $=\mathrm{L} 23^{*} \mathrm{M} 23$ & & & \\
\hline 24 & $\mathrm{Pu}$ & 239 & $=\mathrm{D} 24^{*} \mathrm{~F} 24^{*} \mathrm{~J} 24 / \mathrm{H} 24$ & $=$ Quantities $1 \$ 124$ & $=\mathrm{L} 24^{*} \mathrm{M} 24$ & & & \\
\hline 25 & $\mathrm{Pu}$ & & $=\mathrm{D} 25^{\star} \mathrm{F} 25^{*} \mathrm{~J} 25 / \mathrm{H} 25$ & & & & & \\
\hline 26 & $\mathrm{Pu}$ & 240 & $=D 26^{*} F 26^{*} \mathrm{~J} 26 / \mathrm{H} 26$ & $=$ Quantities $1 \$ 126$ & $=\mathrm{L} 26^{*} \mathrm{M} 26$ & & & \\
\hline 27 & $\mathrm{Pu}$ & 241 & $=\mathrm{D} 27^{*} \mathrm{~F} 27^{*} \mathrm{~J} 27 / \mathrm{H} 27$ & $=$ Quantities $1 \$ 127$ & $=\mathrm{L} 27^{\star} \mathrm{M} 27$ & $=\mathrm{N} 27$ & 120 & $=P 27 / O 27 / 37000000000$ \\
\hline 28 & $\mathrm{Pu}$ & 242 & $=D 28^{*} \mathrm{~F} 28^{*} \mathrm{~J} 28 / \mathrm{H} 28$ & $=$ Quantities $1 \$ 128$ & $=L 28^{*} M 28$ & & & \\
\hline 29 & $\mathrm{Sr}$ & 90 & $=\mathrm{D} 29^{*} \mathrm{~F} 29^{*} \mathrm{~J} 29 / \mathrm{H} 29$ & & & & & \\
\hline 30 & Tc & 99 & & & & & & \\
\hline 31 & Th & 232 & & & & & & \\
\hline 32 & $\mathrm{TI}$ & 204 & & & & & & \\
\hline 33 & $u$ & 235 & & & & & & \\
\hline 34 & $\mathrm{U}$ & 238 & & & & & & \\
\hline
\end{tabular}




\section{Attachment F}

Contamination of sheep spreadsheet calculations 







\begin{tabular}{|c|c|c|c|c|c|c|c|c|}
\hline & A & $\bar{B}$ & $\mathrm{~L}$ & $M$ & $\mathbf{N}$ & 0 & $\mathbf{P}$ & $\mathbf{Q}$ \\
\hline 2 & Sheep & & & & & & & \\
\hline 3 & & & & & & Group & Group DIL & Total \\
\hline 4 & & & & & & sum & & Dep for \\
\hline 5 & & & & & & & $\mathrm{~Bq} / \mathrm{kg}$ & \\
\hline 6 & & & Tsheep & Unit & $T \times$ DEP & & & $\mathrm{Ci} / \mathrm{m} 2$ \\
\hline 7 & & & & Release & $\mathrm{Ci} / \mathbf{k g}$ & Ci/kg & . & \\
\hline 8 & Element & At. No. & $\mathrm{m} 2 / \mathrm{kg}$ & Fraction & & & & \\
\hline 9 & & & & $\mathrm{Ci} / \mathrm{m} 2$ & & & & \\
\hline 10 & Am & 241 & $=\mathrm{D} 10^{*} \mathrm{~F} 10^{*} \mathrm{~J} 10 / \mathrm{H} 10$ & $=$ Quantities $\$ \$ 10$ & $=L 10^{*} M 10$ & & & \\
\hline 11 & Am & 243 & $=\mathrm{D} 11^{*} \mathrm{~F} 11^{*} \mathrm{~J} 11 / \mathrm{H} 11$ & & & & & \\
\hline 12 & $\mathrm{Ba}$ & 133 & & & & & & \\
\hline 13 & $\mathrm{Ce}$ & 144 & $=D 13^{*} \mathrm{~F} 13^{*} \mathrm{~J} 13 / \mathrm{H} 13$ & & & & & \\
\hline 14 & $\mathrm{Cf}$ & 249 & & & & & & \\
\hline 15 & $\mathrm{Cf}$ & 252 & & & Am241. & & & \\
\hline 16 & $\mathrm{Cm}$ & 244 & & & Pu238 & $=N 10+N 23+N 24$ & 2 & $=\mathrm{P} 16 / \mathrm{O} 16 / 37000000000$ \\
\hline 17 & $\mathrm{Cm}$ & 246 & & & Pu239 & & & \\
\hline 18 & Co & 60 & $=\mathrm{D} 18^{*} \mathrm{~F} 18^{\star} \mathrm{J} 18 / \mathrm{H} 18$ & & & & & \\
\hline 19 & Cs & 137 & $=D 19^{*} F 19^{*} \mathrm{~J} 19 / \mathrm{H} 19$ & & & & & \\
\hline 20 & H & 3 & & & & & & \\
\hline 21 & $\mathrm{~Np}$ & 237 & & & & & & \\
\hline 22 & $\mathrm{Pm}$ & 147 & & & & & & \\
\hline 23 & $\mathrm{Pu}$ & 238 & $=\mathrm{D} 23^{*} \mathrm{~F} 23^{*} \mathrm{~J} 23 / \mathrm{H} 23$ & $=$ Quantities $! \$ 123$ & $=\mathrm{L} 23^{*} \mathrm{M} 23$ & & & \\
\hline 24 & $\mathrm{Pu}$ & 239 & $=\mathrm{D} 24^{*} \mathrm{~F} 24^{*} \mathrm{~J} 24 / \mathrm{H} 24$ & $=$ Quantities $1 \$ 124$ & $=\mathrm{L} .24^{\star} \mathrm{M} 24$ & & & \\
\hline 25 & $\mathrm{Pu}$ & & $=\mathrm{D} 25^{*} \mathrm{~F} 25^{*} \mathrm{~J} 25 / \mathrm{H} 25$ & & & & & \\
\hline 26 & $\mathrm{Pu}$ & 240 & $=\mathrm{D} 26^{*} \mathrm{~F} 26^{*} \mathrm{~J} 26 / \mathrm{H} 26$ & $=$ Quantities $1 \$ 126$ & $=\mathrm{L} 26^{*} \mathrm{M} 26$ & & & \\
\hline 27 & $\mathrm{Pu}$ & 241 & $=\mathrm{D} 27^{\star} \mathrm{F} 27^{*} \mathrm{~J} 27 / \mathrm{H} 27$ & $=$ Quantities $1 \$ 127$ & $=L 27 * M 27$ & $=\mathrm{N} 27$ & 120 & $=\mathrm{P} 27 / \mathrm{O} 27 / 37000000000$ \\
\hline 28 & $\mathrm{Pu}$ & 242 & $=\mathrm{D} 28^{*} \mathrm{~F} 28^{*} \mathrm{~J} 28 / \mathrm{H} 28$ & $=$ Quantities $1 \$ 128$ & $=\mathrm{L} 28^{*} \mathrm{M} 28$ & & & \\
\hline 29 & $\mathrm{Sr}$ & 90 & $=\mathrm{D} 29^{*} \mathrm{~F} 29^{*} \mathrm{~J} 29 / \mathrm{H} 29$ & & & & & \\
\hline 30 & Tc & 99 & & & & & & \\
\hline 31 & Th & 232 & & & & & & \\
\hline 32 & $\mathrm{TI}$ & 204 & & & & & & \\
\hline 33 & $\mathrm{u}$ & 235 & & & & & & \\
\hline 34 & u & 238 & & & & & & \\
\hline
\end{tabular}


Attachment G

Contamination of lamb spreadsheet calculations 







\begin{tabular}{|c|c|c|c|c|c|c|c|c|}
\hline & $\mathrm{A}$ & $\bar{B}$ & $L$ & $M$ & $\mathbf{N}$ & 0 & $\mathbf{P}$ & $Q$ \\
\hline 2 & Lamb & & & & & & & \\
\hline 3 & & & & & & Group & Group DIL & Total \\
\hline 4 & & & & & & sum & & Dep for \\
\hline 5 & & & & & & & $\mathrm{~Bq} / \mathrm{kg}$ & DIL \\
\hline 6 & & & Tlamb & Unit & $T \times$ DEP & & & $\mathrm{Ci} / \mathrm{m} 2$ \\
\hline 7 & & & & Release & $\mathrm{Ci} / \mathrm{kg}$ & $\mathrm{Ci} / \mathrm{kg}$ & " & \\
\hline 8 & Element & At. No. & $\mathrm{m} 2 / \mathrm{kg}$ & Fraction & & & & \\
\hline 9 & & & & $\mathrm{Ci} / \mathrm{m} 2$ & & & & \\
\hline 10 & Am & 241 & $=\mathrm{D} 10^{*} \mathrm{~F} 10^{*} \mathrm{~J} 10 / \mathrm{H} 10$ & $=$ Quantities $1 \$ 110$ & $=L 10^{*} \mathrm{M} 10$ & & & \\
\hline 11 & Am & 243 & $=\mathrm{D} 11^{*} \mathrm{~F} 11^{*} \mathrm{~J} 11 / \mathrm{H} 11$ & & & & & \\
\hline 12 & $B a$ & 133 & & & & & & \\
\hline 13 & $\mathrm{Ce}$ & 144 & & & & & & \\
\hline 14 & Cf & 249 & & & & & & \\
\hline 15 & Cf & 252 & & & Am241 & & & \\
\hline 16 & $\mathrm{Cm}$ & 244 & & & Pu238 & $=N 10+N 23+N 24$ & 2 & $=\mathrm{P} 16 / 016 / 37000000000$ \\
\hline 17 & $\mathrm{Cm}$ & 246 & & & Ru239 & & & \\
\hline 18 & Co & 60 & & & & & & \\
\hline 19 & Cs & 137 & $=\mathrm{D} 19^{*} \mathrm{~F} 19^{*} \mathrm{~J} 19 / \mathrm{H} 19$ & & & & & \\
\hline 20 & $H$ & 3 & & & & & & \\
\hline 21 & Np & 237 & & & & & & \\
\hline 22 & Pm & 147 & & & & & & \\
\hline 23 & $\mathrm{Pu}$ & 238 & $=D 23^{*} F 23^{*} \mathrm{~J} 23 / \mathrm{H} 23$ & $=$ Quantities $1 \$ 123$ & $=\mathrm{L} 23^{\star} \mathrm{M} 23$ & & & \\
\hline 24 & $\mathrm{Pu}$ & 239 & $=D 24^{*} \mathrm{~F} 24^{*} \mathrm{~J} 24 / \mathrm{H} 24$ & $=$ Quantities $1 \$ 124$ & $=L 24^{*} M 24$ & & & \\
\hline 25 & $\mathrm{Pu}$ & & $=\mathrm{D} 25^{\star} \mathrm{F} 25^{*} \mathrm{~J} 25 / \mathrm{H} 25$ & & & & & \\
\hline 26 & $\mathrm{Pu}$ & 240 & $=\mathrm{D} 26^{*} \mathrm{~F} 26^{*} \mathrm{~J} 26 / \mathrm{H} 26$ & $=$ Quantities!\$126 & $=\mathrm{L} 26^{*} \mathrm{M} 26$ & & & \\
\hline 27 & $\mathrm{Pu}$ & 241 & $=\mathrm{D} 27^{*} \mathrm{~F} 27^{*} \mathrm{~J} 27 / \mathrm{H} 27$ & $=$ Quantities $1 \$ 127$ & $=\mathrm{L} 27^{*} \mathrm{M} 27$ & $=N 27$ & 120 & $=\mathrm{P} 27 / \mathrm{O} 27 / 37000000000$ \\
\hline 28 & $\mathrm{Pu}$ & 242 & $=\mathrm{D} 28^{*} \mathrm{~F} 28^{*} \mathrm{~J} 28 / \mathrm{H} 28$ & $=$ Quantities $1 \$ 128$ & $=L 28^{*} M 28$ & & & \\
\hline 29 & $\mathrm{Sr}$ & 90 & $=\mathrm{D} 29^{*} \mathrm{~F} 29^{*} \mathrm{~J} 29 / \mathrm{H} 29$ & & & & & \\
\hline 30 & Tc & 99 & & & & & & \\
\hline 31 & Th & 232 & & & & & & \\
\hline 32 & $\mathrm{TI}$ & 204 & & & & & & \\
\hline 33 & $u$ & 235 & & & & & & \\
\hline 34 & $u$ & 238 & & & & & & \\
\hline
\end{tabular}


Attachment $H$

Contamination of pork spreadsheet calculations 


\begin{tabular}{|c|c|c|c|c|c|c|c|c|}
\hline & A & $\bar{B}$ & $L$ & $\bar{M}$ & $\bar{N}$ & 0 & $\overline{\mathbf{P}}$ & $\bar{Q}$ \\
\hline 2 & Pork & & & & & & & \\
\hline 3 & & & & & & Group & Group DIL & Total \\
\hline 4 & & & & & & sum & & Dep for \\
\hline 5 & & & & & & & $\mathrm{~Bq} / \mathrm{kg}$ & DIL \\
\hline 6 & & & Tpork & Unit & $T \times$ DEP & & & $\mathrm{Ci} / \mathrm{m} 2$ \\
\hline 7 & & & & Release & Ci/kg & Ci/kg & & \\
\hline 8 & Element & At. No. & $\mathrm{m} 2 / \mathrm{kg}$ & Fraction & & & & \\
\hline 9 & & & & $\mathrm{Ci} / \mathrm{m} 2$ & & & & \\
\hline 10 & Am & 241 & 5.19E-04 & 1.87E-02 & 9.71E-06 & & & \\
\hline 11 & Am & 243 & 5.19E-04 & & & & & \\
\hline 12 & $\mathrm{Ba}$ & 133 & $0.00 E+00$ & & & & & \\
\hline 13 & $\mathrm{Ce}$ & 144 & 3.06E-04 & & & & & \\
\hline 14 & Cf & 249 & & & & & & \\
\hline 15 & Cf & 252 & & & Am241 & & & \\
\hline 16 & $\mathrm{Cm}$ & 244 & & & Pu238 & $2.10 \mathrm{E}-05$ & 2 & 2.57E-06 \\
\hline 17 & $\mathrm{Cm}$ & 246 & & & Pu239 & & & \\
\hline 18 & Co & 60 & & & & & & \\
\hline 19 & Cs & 137 & 7.33E-01 & & & & & \\
\hline 20 & H & 3 & & & & & & \\
\hline 21 & Np & 237 & & & & & & \\
\hline 22 & $\mathrm{Pm}$ & 147 & & & & & & \\
\hline 23 & $\mathrm{Pu}$ & 238 & 2.44E-04 & 4.61E-02 & 1.13E-05 & & & \\
\hline 24 & Pu & 239 & 2.44E-04 & $1.20 E-04$ & 2.94E-08 & & & \\
\hline 25 & $\mathrm{Pu}$ & $39 / 241$ & $2.44 E-04$ & & & & & \\
\hline 26 & $\mathrm{Pu}$ & 240 & 2.44E-04 & $6.92 E-03$ & 1.69E-06 & & & \\
\hline 27 & $\mathrm{Pu}$ & 241 & $2.44 E-04$ & $9.28 \mathrm{E}-01$ & 2.27E-04 & 2.27E-04 & 120 & 1.43E-05 \\
\hline 28 & $\mathrm{Pu}$ & 242 & 2.44E-04 & 4.38E-04 & 1.07E-07 & & & \\
\hline 29 & $\mathrm{Sr}$ & 90 & 1.22E-01 & & & & & \\
\hline 30 & Tc & 99 & 4.58E-04 & & & & & \\
\hline 31 & Th & 232 & & & & & & \\
\hline 32 & $\mathrm{Tl}$ & 204 & & & & & & \\
\hline 33 & $\mathrm{u}$ & 235 & $1.89 E-01$ & & & & & \\
\hline 34 & $\mathbf{u}$ & 238 & 1.89E-01 & & & & & \\
\hline
\end{tabular}







Attachment I

Contamination of poultry spreadsheet calculations 


\begin{tabular}{|c|c|c|c|c|c|c|c|c|}
\hline & $\bar{A}$ & B & $L$ & $M$ & $\mathbf{N}$ & 0 & $\bar{P}$ & $\mathbf{Q}$ \\
\hline 2 & \multicolumn{8}{|l|}{ Poultry } \\
\hline 3 & \multirow{7}{*}{ Element } & \multirow{7}{*}{ At. No. } & \multirow{7}{*}{ Tpoultry } & \multirow{7}{*}{$\begin{array}{l}\text { Unit } \\
\text { Release } \\
\text { Fraction } \\
\text { Ci/m2 }\end{array}$} & \multirow{5}{*}{$\begin{array}{l}T \times \text { DEP } \\
\mathrm{Ci} / \mathrm{kg}\end{array}$} & \multirow{4}{*}{$\begin{array}{l}\text { Group } \\
\text { sum }\end{array}$} & Group DIL & Total \\
\hline 4 & & & & & & & & Dep for \\
\hline 5 & & & & & & & $\mathrm{~Bq} / \mathrm{kg}$ & DIL \\
\hline 6 & & & & & & & & $\mathrm{Ci} / \mathrm{m} 2$. \\
\hline 7 & & & & & & $\mathrm{Ci} / \mathrm{kg}$ & & \\
\hline 8 & & & & & & & & \\
\hline 9 & & & & & & & & \\
\hline 10 & Am & 241 & $5.25 E-04$ & 1.87E-02 & $9.82 E-06$ & & & \\
\hline 11 & Am & 243 & $5.25 \mathrm{E}-04$ & & & & & \\
\hline 12 & $\mathrm{Ba}$ & 133 & 7.88E-04 & & & & & \\
\hline 13 & $\mathrm{Ce}$ & 144 & 3.50E-04 & & & & & \\
\hline 14 & Cf & 249 & & & & & & \\
\hline 15 & $\mathrm{Cf}$ & 252 & & & Am241 & & & \\
\hline 16 & $\mathrm{Cm}$ & 244 & & & Pu238 & $2.19 E-05$ & 2 & 2.46E-06 \\
\hline 17 & $\mathrm{Cm}$ & 246 & & & Pu239 & & & \\
\hline 18 & Co & 60 & 1.75E-01 & & & & & \\
\hline 19 & Cs & 137 & $8.75 E-01$ & & & & & \\
\hline 20 & $H$ & 3 & & & & & & \\
\hline 21 & $N p$ & 237 & & & & & & \\
\hline 22 & Pm & 147 & $1.75 E-04$ & & & & & \\
\hline 23 & $\mathrm{Pu}$ & 238 & 2.63E-04 & 4.61E-02 & $1.21 \mathrm{E}-05$ & & & \\
\hline 24 & $\mathrm{Pu}$ & 239 & 2.63E-04 & 1.20E-04 & $3.16 E-08$ & & & \\
\hline 25 & $\mathrm{Pu}$ & $239 / 241$ & 2.63E-04 & & & & & \\
\hline 26 & $\mathrm{Pu}$ & 240 & 2.63E-04 & $6.92 \mathrm{E}-03$ & $1.82 E-06$ & & & \\
\hline 27 & $\mathrm{Pu}$ & 241 & 2.63E-04 & 9.28E-01 & 2.44E-04 & 2.44E-04 & 120 & 1.33E-05 \\
\hline 28 & $\mathrm{Pu}$ & 242 & 2.63E-04 & 4.38E-04 & 1.15E-07 & & & \\
\hline 29 & $\mathrm{Sr}$ & 90 & $7.00 \mathrm{E}-03$ & & & & & \\
\hline 30 & Tc & 99 & 2.63E-03 & & & & & \\
\hline 31 & Th & 232 & & & & & & \\
\hline 32 & $\mathrm{TI}$ & 204 & & & & & & \\
\hline 33 & $u$ & 235 & 8.75E-02 & & & & & \\
\hline 34 & $\mathrm{u}$ & 238 & $8.75 \mathrm{E}-02$ & & & & & \\
\hline
\end{tabular}




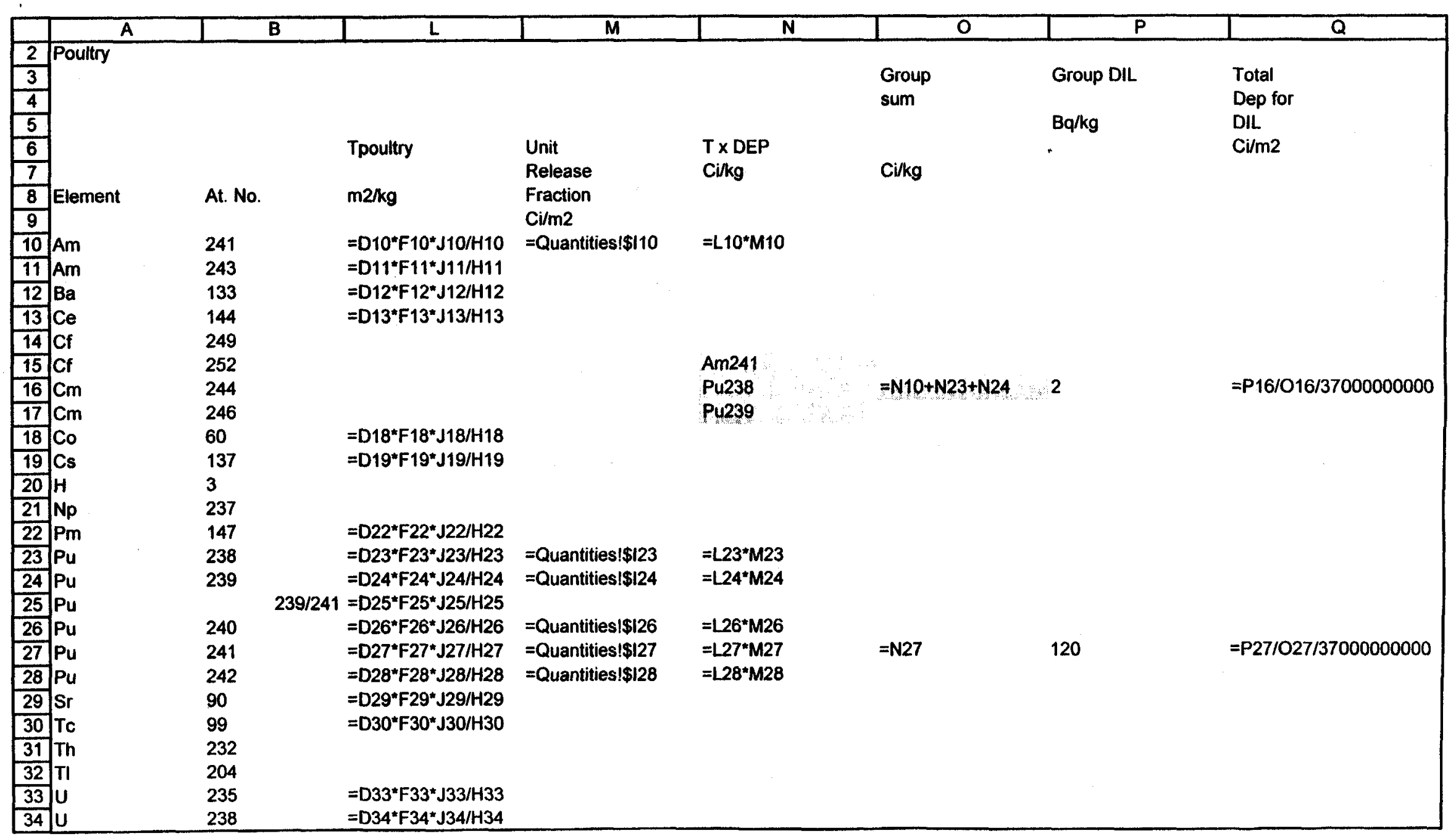




\section{Attachment J}

Contamination of fish spreadsheet calculations 


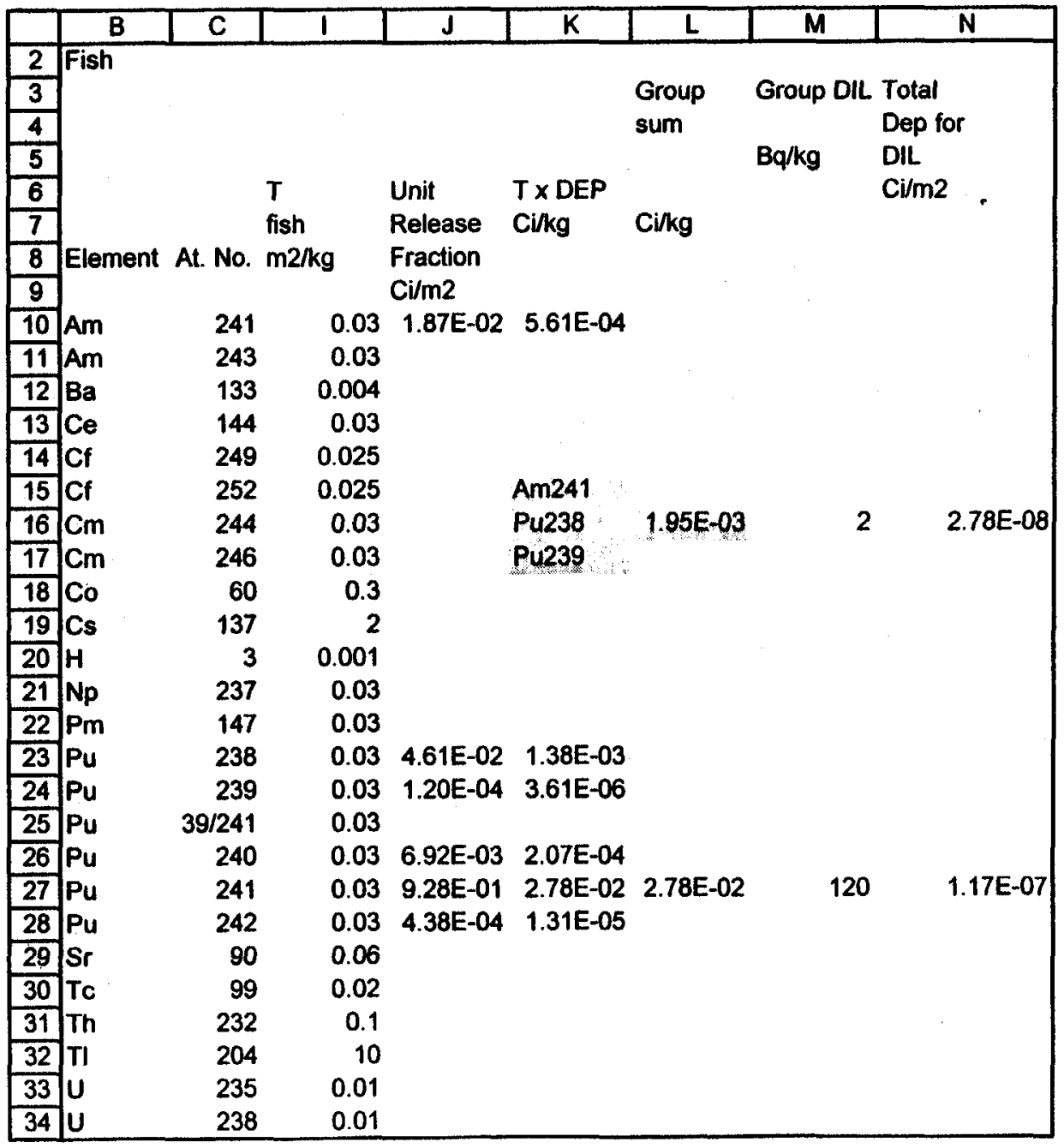




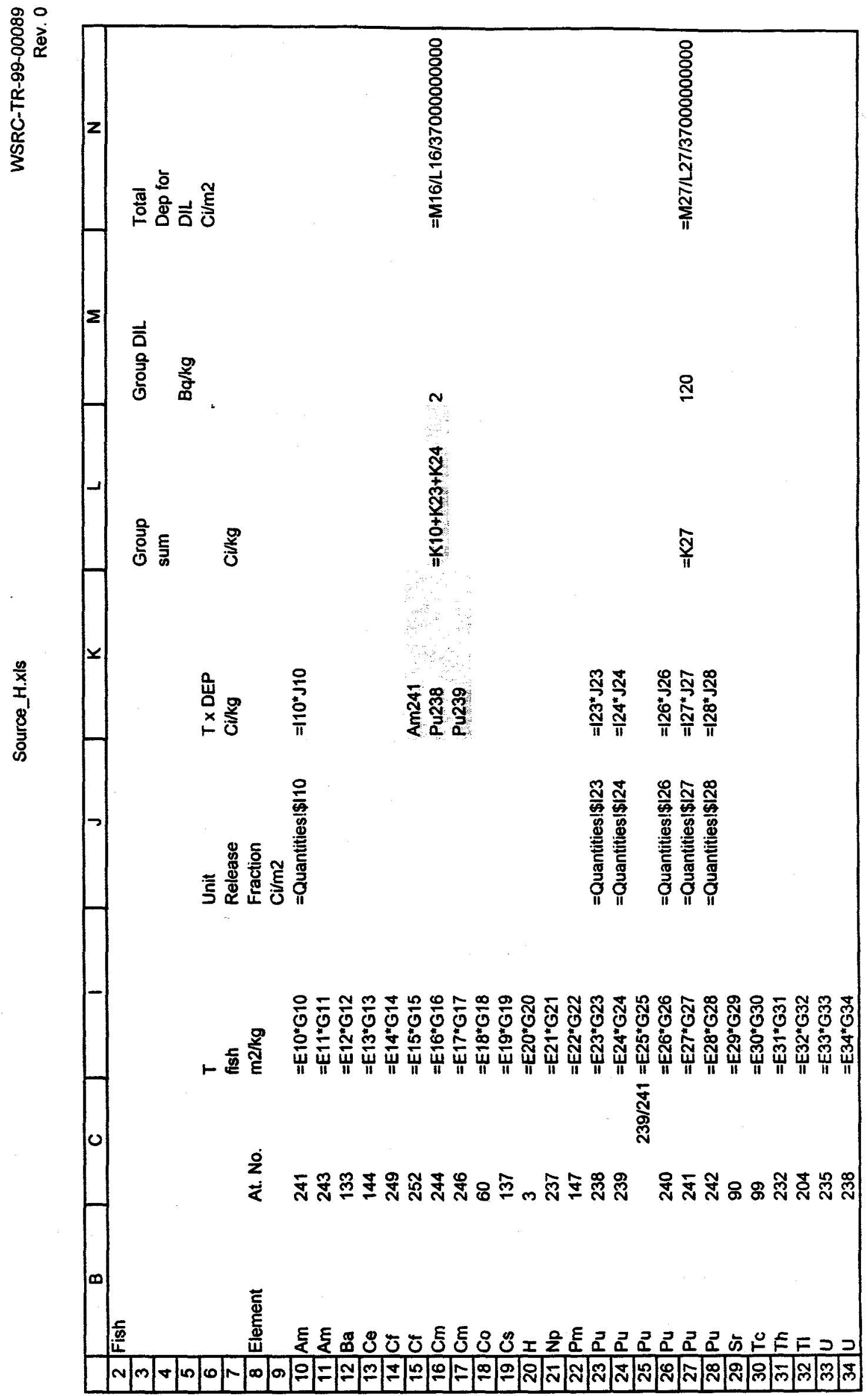


Attachment K

External contamination of produce from direct deposition spreadsheet calculations 


\begin{tabular}{|c|c|c|c|c|c|c|c|c|}
\hline & $\bar{A}$ & B & $\mathrm{H}$ & T & $J$ & $\bar{K}$ & $\bar{L}$ & $\bar{M}$ \\
\hline 2 & \multicolumn{8}{|c|}{ Produce-direct deposition } \\
\hline 3 & & & & & & Group & Group DIL & Total \\
\hline 4 & & & & & & sum & & Dep for \\
\hline 5 & & & $\mathrm{~m} 2 / \mathrm{kg}$ & & & & $\mathrm{Bq} / \mathrm{kg}$ & DIL \\
\hline 6 & & & & Unit & $T \times D E P$ & & & $\mathrm{Ci} / \mathrm{m} 2$ \\
\hline 7 & & & & Release & $\mathrm{Ci} / \mathrm{kg}$ & $\mathrm{Ci} / \mathrm{kg}$ & & \\
\hline 8 & Element & At. No. & $T$ & Fraction & & & & \\
\hline 9 & & & & $\mathrm{Ci} / \mathrm{m} 2$ & & & & \\
\hline 10 & Am & 241 & 0.285714 & 1.87E-02 & 5.34E-03 & & & \\
\hline 11 & Am & 243 & 0.285714 & & & & & \\
\hline 12 & $\mathrm{Ba}$ & 133 & 0.285714 & & & & & \\
\hline 13 & $\mathrm{Ce}$ & 144 & 0.285714 & & & & & \\
\hline 14 & Cf & 249 & 0.285714 & & & & & \\
\hline 15 & Cf & 252 & 0.285714 & & Am241 & & & \\
\hline 16 & $\mathrm{Cm}$ & 244 & 0.285714 & & Pu238 & $1.85 E-02$ & 2 & 2.91E-09 \\
\hline 17 & $\mathrm{Cm}$ & 246 & 0.285714 & & Pu239 & & & \\
\hline 18 & Co & 60 & 0.285714 & & & & & \\
\hline 19 & Cs & 137 & 0.285714 & & & & & \\
\hline 20 & $H$ & 3 & 0.285714 & & & & & \\
\hline$\overline{21}$ & Np & 237 & 0.285714 & & & & & \\
\hline 22 & $\mathrm{Pm}$ & 147 & 0.285714 & & & & & \\
\hline 23 & $\mathrm{Pu}$ & 238 & 0.285714 & 4.61E-02 & 1.32E-02 & & & \\
\hline 24 & $\mathrm{Pu}$ & 239 & 0.285714 & 1.20E-04 & 3.44E-05 & & & \\
\hline 25 & $\mathrm{Pu}$ & $239 / 241$ & 0.285714 & & & & & \\
\hline 26 & $\mathrm{Pu}$ & 240 & 0.285714 & 6.92E-03 & $1.98 E-03$ & & & \\
\hline 27 & $\mathrm{Pu}$ & 241 & 0.285714 & $9.28 E-01$ & $2.65 E-01$ & 2.65E-01 & 120 & 1.22E-08 \\
\hline 28 & $\mathrm{Pu}$ & 242 & 0.285714 & 4.38E-04 & 1.25E-04 & & & \\
\hline 29 & $\mathrm{sr}$ & 90 & 0.285714 & & & & & \\
\hline 30 & Tc & 99 & 0.285714 & & & & & \\
\hline 31 & Th & 232 & 0.285714 & & & & & \\
\hline 32 & $\mathrm{TI}$ & 204 & 0.285714 & & & & & \\
\hline 33 & $u$ & 235 & 0.285714 & & & & & \\
\hline 34 & $\mathrm{u}$ & 238 & 0.285714 & & & & & \\
\hline
\end{tabular}









\section{Attachment L}

Internal contamination of produce from root uptake spreadsheet calculations 


\begin{tabular}{|c|c|c|c|c|c|c|c|c|}
\hline & $\bar{A}$ & $\mathbf{B}$ & $\bar{H}$ & 1 & $J$ & $\mathbf{K}$ & $\mathbf{L}$ & $\mathbf{M}$ \\
\hline 2 & \multicolumn{8}{|c|}{ Produce-root uptake } \\
\hline 3 & & & & & & Group & \multirow{2}{*}{\multicolumn{2}{|c|}{$\begin{array}{l}\text { Group Cotal } \\
\text { Dep for }\end{array}$}} \\
\hline 4 & & & & & & sum & & \\
\hline 5 & & & & & & & $\mathrm{~Bq} / \mathrm{kg}$ & DIL \\
\hline 6 & & & m2/kg & Unit & $T \times D E P$ & & & $\mathrm{Ci} / \mathrm{m} 2$. \\
\hline 7 & & & & Release & Ci/kg & Ci/kg & & \\
\hline 8 & Element & At. No. & $T=B / 280$ & Fraction & & & & \\
\hline 9 & & & & $\mathrm{Ci} / \mathrm{m} 2$ & & & & \\
\hline 10 & Am & 241 & 3.37E-07 & 1.87E-02 & 6.30E-09 & & & \\
\hline 11 & Am & 243 & 3.37E-07 & & & & & \\
\hline 12 & $\mathrm{Ba}$ & 133 & $1.53 E-05$ & & & & & \\
\hline 13 & $\mathrm{Ce}$ & 144 & 1.53E-05 & & & & & \\
\hline 14 & $\mathrm{Cf}$ & 249 & $0.00 E+00$ & & & & & \\
\hline 15 & $\mathrm{Cf}$ & 252 & $0.00 E+00$ & & Am241 & & & \\
\hline 16 & $\mathrm{Cm}$ & 244 & 3.93E-07 & & Pu238 & $8.02 E-09$ & 2 & $6.74 E-03$ \\
\hline 17 & $\mathrm{Cm}$ & 246 & $3.93 E-07$ & & Pu239 & & & \\
\hline 18 & Co & 60 & $1.02 E-04$ & & & & & \\
\hline$\overline{19}$ & Cs & 137 & & & & & & \\
\hline 20 & $H$ & 3 & & & & & & \\
\hline 21 & $\mathrm{~Np}$ & 237 & 1.89E-05 & & & & & \\
\hline 22 & $\mathrm{Pm}$ & 147 & $0.00 E+00$ & & & & & \\
\hline 23 & Pu & 238 & 3.72E-08 & 4.61E-02 & 1.72E-09 & & & \\
\hline 24 & $\mathrm{Pu}$ & 239 & 3.72E-08 & $1.20 E-04$ & $4.48 E-12$ & & & \\
\hline 25 & $\mathrm{Pu}$ & $39 / 241$ & 3.72E-08 & & & & & \\
\hline 26 & $\mathrm{Pu}$ & 240 & 3.72E-08 & $6.92 E-03$ & 2.58E-10 & & & \\
\hline 27 & $\mathrm{Pu}$ & 241 & 3.72E-08 & $9.28 E-01$ & $3.46 \mathrm{E}-08$ & $3.46 E-08$ & 120 & 9.39E-02 \\
\hline 28 & $\mathrm{Pu}$ & 242 & 3.72E-08 & 4.38E-04 & $1.63 \mathrm{E}-11$ & & & \\
\hline 29 & Sr & 90 & & & & & & \\
\hline 30 & Tc & 99 & $1.02 \mathrm{E}-01$ & & & & & \\
\hline 31 & Th & 232 & $9.18 \mathrm{E}-07$ & & & & & \\
\hline 32 & $\mathrm{TI}$ & 204 & $0.00 E+00$ & & & & & \\
\hline 33 & $\mathrm{U}$ & 235 & 4.23E-06 & & & & & \\
\hline 34 & u & 238 & 4.23E-06 & & & & & \\
\hline
\end{tabular}




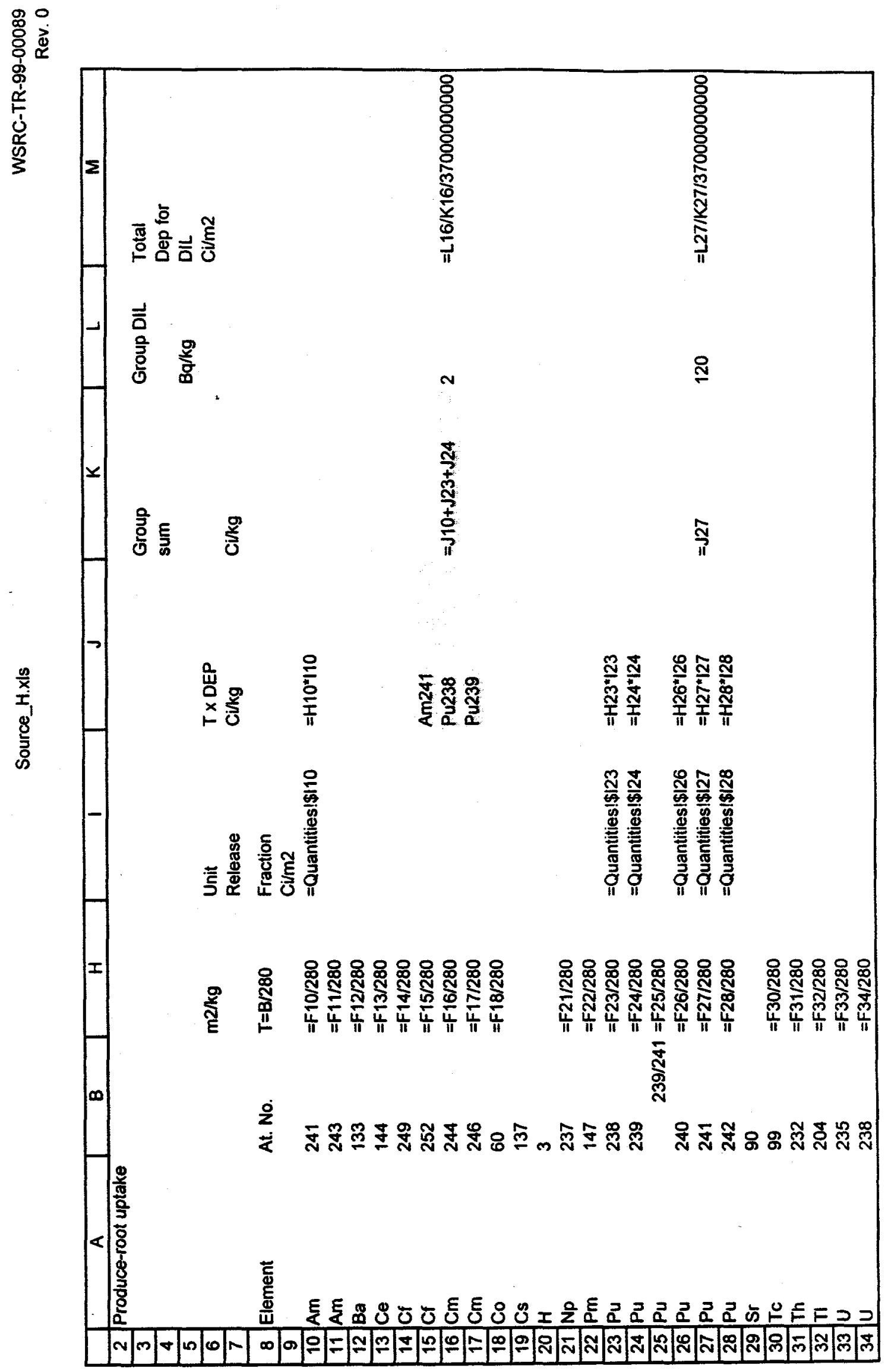

บ 
Attachment $\mathbf{M}$

External contamination of leafy vegetables during harvest spreadsheet calculations 


\begin{tabular}{|c|c|c|c|c|c|c|c|c|}
\hline & A & $\bar{B}$ & $\mathrm{H}$ & T & $J$ & $\bar{K}$ & $\mathrm{~L}$ & $\mathbf{M}$ \\
\hline 2 & \multicolumn{8}{|c|}{ Produce-soil adhesion } \\
\hline 3 & & & & & & Group & Group DIL & Total \\
\hline 4 & & & & & & sum & & Dep for \\
\hline 5 & & & & & & & $\mathrm{~Bq} / \mathrm{kg}$ & DIL \\
\hline 6 & & & & Unit & $T \times$ DEP & & & Ci/m2 \\
\hline 7 & & & & Release & Ci/kg & $\mathrm{Ci} / \mathrm{kg}$ & & \\
\hline 8 & Element & At. No. & $T=D x F / 280$ & Fraction & & & & \\
\hline 9 & & & & $\mathrm{Ci} / \mathrm{m} 2$ & & & & \\
\hline 10 & Am & 241 & 5.10204E-06 & 1.87E-02 & $9.54 E-08$ & & & \\
\hline 11 & Am & 243 & 5.10204E-06 & & & & & \\
\hline 12 & $\mathrm{Ba}$ & 133 & 5.10204E-06 & & & & & \\
\hline 13 & $\mathrm{Ce}$ & 144 & 5.10204E-06 & & & & & \\
\hline 14 & $\mathrm{Cf}$ & 249 & 5.10204E-06 & & & & & \\
\hline 15 & Cf & 252 & 5.10204E-06 & & Am241 & & & \\
\hline 16 & $\mathrm{Cm}$ & 244 & 5.10204E-06 & & Pu238 & $3.31 E-07$ & 2 & 1.63E-04 \\
\hline 17 & $\mathrm{Cm}$ & 246 & 5.10204E-06 & - & Pu239 & & & \\
\hline 18 & Co & 60 & 5.10204E-06 & & & & & \\
\hline 19 & Cs & 137 & 5.10204E-06 & & & & & \\
\hline 20 & $\mathrm{H}$ & 3 & 5.10204E-06 & & & & & \\
\hline 21 & $\mathrm{~Np}$ & 237 & 5.10204E-06 & & & & & \\
\hline 22 & $\mathrm{Pm}$ & 147 & 5. $10204 E-06$ & & & & & \\
\hline 23 & $\mathrm{Pu}$ & 238 & 5. 10204E-06 & 4.61E-02 & 2.35E-07 & & & \\
\hline 24 & $\mathrm{Pu}$ & 239 & $5.10204 E-06$ & $1.20 \mathrm{E}-04$ & $6.14 E-10$ & & & \\
\hline 25 & $\mathrm{Pu}$ & $239 / 241$ & 5.10204E-06 & & & & & \\
\hline 26 & $\mathrm{Pu}$ & 240 & 5.10204E-06 & $6.92 \mathrm{E}-03$ & 3.53E-08 & & & \\
\hline 27 & $\mathrm{Pu}$ & 241 & 5.10204E-06 & $9.28 E-01$ & 4.73E-06 & 4.73E-06 & 120 & 6.85E-04 \\
\hline 28 & $\mathrm{Pu}$ & 242 & 5.10204E-06 & 4.38E-04 & 2.24E-09 & & & \\
\hline 29 & $\mathrm{Sr}$ & 90 & 5.10204E-06 & & & & & \\
\hline 30 & Tc & 99 & 5.10204E-06 & & & & & \\
\hline 31 & Th & 232 & 5.10204E-06 & & & & & \\
\hline 32 & $\mathrm{TI}$ & 204 & 5.10204E-06 & & & & & \\
\hline 33 & $\mathrm{u}$ & 235 & 5. 10204E-06 & & & & & \\
\hline 34 & $\mathrm{U}$ & 238 & 5.10204E-06 & & & & & \\
\hline
\end{tabular}




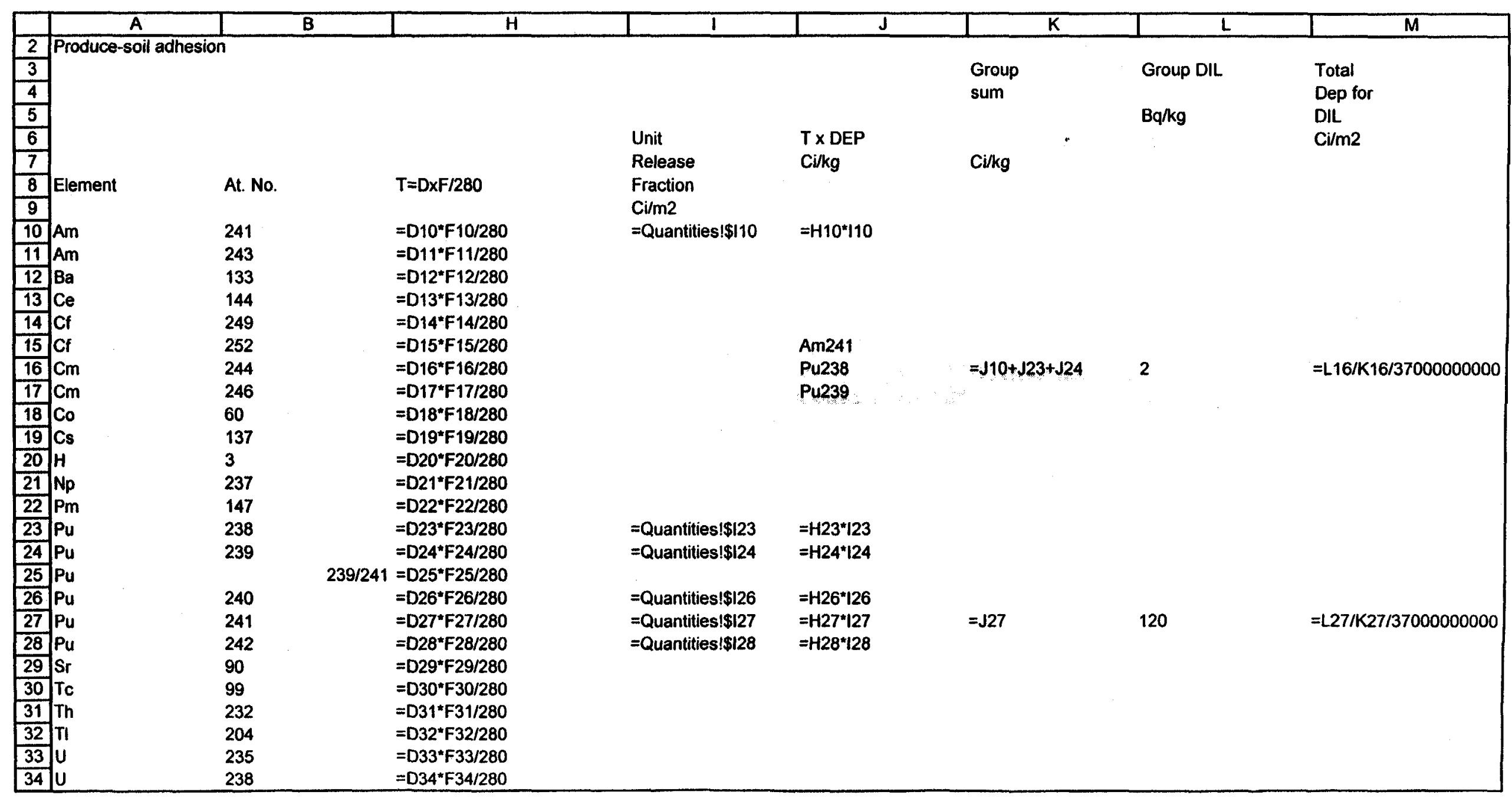


Attachment N

External contamination of grain spreadsheet calculations 


\begin{tabular}{|c|c|c|c|c|c|c|c|c|}
\hline & $\bar{A}$ & $\bar{B}$ & H & 1 & $\mathrm{~J}$ & $\bar{K}$ & $L$ & $\mathbf{M}$ \\
\hline 2 & \multicolumn{8}{|c|}{ Grain-direct deposition } \\
\hline 3 & & & & & & Group & Group DIL & Total \\
\hline 4 & & & & & & sum & & Dep for \\
\hline 5 & & & $\mathrm{~m} 2 / \mathrm{kg}$ & & & & $\mathrm{Bq} / \mathrm{kg}$ & DIL \\
\hline 6 & & & & Unit & $T \times D E P$ & & & $\mathrm{Ci} / \mathrm{m}_{2}$ \\
\hline 7 & & & & Release & $\mathrm{Ci} / \mathrm{kg}$ & Ci/kg & & \\
\hline 8 & Element & At. No. & $\mathbf{T}$ & Fraction & & & & \\
\hline 9 & & & & $\mathrm{Ci} / \mathrm{m} 2$ & & & & \\
\hline 10 & Am & 241 & 0.285714 & 1.87E-02 & 5.34E-03 & & & \\
\hline 11 & Am & 243 & 0.285714 & & & & & \\
\hline 12 & Ba & 133 & 0.285714 & & & & & \\
\hline 13 & $\mathrm{Ce}$ & 144 & 0.285714 & & & & & \\
\hline 14 & Cf & 249 & 0.285714 & & & & & \\
\hline 15 & Cf & 252 & 0.285714 & & Am241 & & & \\
\hline 16 & $\mathrm{Cm}$ & 244 & 0.285714 & & Pu238 & $1.85 E-02$ & 2 & 2.91E-09 \\
\hline 17 & $\mathrm{Cm}$ & 246 & 0.285714 & & Pu239 & & & \\
\hline 18 & Co & 60 & 0.285714 & & & & & \\
\hline 19 & Cs & 137 & 0.285714 & & & & & \\
\hline 20 & $H$ & 3 & 0.285714 & & & & & \\
\hline 21 & Np & 237 & 0.285714 & & & & & \\
\hline 22 & $\mathrm{Pm}$ & 147 & 0.285714 & & & & & \\
\hline 23 & $\mathrm{Pu}$ & 238 & 0.285714 & 4.61E-02 & 1.32E-02 & & & \\
\hline 24 & $\mathrm{Pu}$ & 239 & 0.285714 & 1.20E-04 & $3.44 E-05$ & & & \\
\hline 25 & $\mathrm{Pu}$ & $239 / 241$ & 0.285714 & & & & & \\
\hline 26 & $\mathrm{Pu}$ & 240 & 0.285714 & $6.92 E-03$ & $1.98 E-03$ & & & \\
\hline 27 & $\mathrm{Pu}$ & 241 & 0.285714 & 9.28E-01 & $2.65 \mathrm{E}-01$ & 2.65E-01 & 120 & 1.22E-08 \\
\hline 28 & $\mathrm{Pu}$ & 242 & 0.285714 & 4.38E-04 & 1.25E-04 & & & \\
\hline 29 & $\mathrm{Sr}$ & 90 & 0.285714 & & & & & \\
\hline 30 & Tc & 99 & 0.285714 & & & & & \\
\hline 31 & Th & 232 & 0.285714 & & & & & \\
\hline 32 & $\mathrm{TI}$ & 204 & 0.285714 & & & & & \\
\hline 33 & u & 235 & 0.285714 & & & & & \\
\hline 34 & $u$ & 238 & 0.285714 & & & & & \\
\hline
\end{tabular}







Attachment $\mathbf{O}$

Internal contamination of grain by root uptake spreadsheet calculations 


\begin{tabular}{|c|c|c|c|c|c|c|c|c|}
\hline & $\bar{A}$ & B & $\bar{H}$ & 1 & $\mathrm{~J}$ & $\mathbf{K}$ & $\mathbf{L}$ & $\bar{M}$ \\
\hline 2 & \multicolumn{8}{|c|}{ Grain-root uptake } \\
\hline 3 & & & & & & Group & Group DIL & Total \\
\hline 4 & & & & & & sum & & Dep for \\
\hline 5 & & & & & & & $\mathrm{~Bq} / \mathrm{kg}$ & DIL \\
\hline 6 & & & $\mathrm{~m} 2 / \mathrm{kg}$ & Unit & $T \times D E P$ & & & $\mathrm{Ci} / \mathrm{m}_{2}$ \\
\hline 7 & & & & Release & Ci/kg & $\mathrm{Ci} / \mathrm{kg}$ & & \\
\hline 8 & Element & At. No. & $T=B / 280$ & Fraction & & & & \\
\hline 9 & & & & $\mathrm{Ci} / \mathrm{m} 2$ & & & & \\
\hline 10 & Am & 241 & $6.76 E-08$ & 1.87E-02 & $1.26 E-09$ & & & \\
\hline 11 & Am & 243 & $6.76 E-08$ & & & & & \\
\hline 12 & $\mathrm{Ba}$ & 133 & 9.21E-05 & & & & & \\
\hline 13 & $\mathrm{Ce}$ & 144 & 9.21E-05 & & & & & \\
\hline 14 & Cf & 249 & & & & & & \\
\hline 15 & $\mathrm{Cf}$ & 252 & & & Am241 & & & \\
\hline 16 & $\mathrm{Cm}$ & 244 & $6.45 \mathrm{E}-08$ & & Pu238 & 2.48E-09 & 2 & 2.18E-02 \\
\hline 17 & $\mathrm{~cm}$ & 246 & 6.45E-08 & & Pu239 & & & \\
\hline 18 & Co & 60 & 1.14E-05 & & & & & \\
\hline 19 & Cs & 137 & & & & & & \\
\hline 20 & $H$ & 3 & & & & & & \\
\hline 21 & Np & 237 & 8.29E-06 & & & & & \\
\hline 22 & Pm & 147 & & & & & & \\
\hline 23 & $\mathrm{Pu}$ & 238 & $2.64 E-08$ & $4.61 E-02$ & $1.22 E-09$ & & & \\
\hline 24 & $\mathrm{Pu}$ & 239 & 2.64E-08 & 1.20E-04 & 3.18E-12 & & & \\
\hline 25 & $P u$ & $239 / 241$ & 2.64E-08 & & & & & \\
\hline 26 & $\mathrm{Pu}$ & 240 & 2.64E-08 & 6.92E-03 & $1.83 E-10$ & & & \\
\hline 27 & $\mathrm{Pu}$ & 241 & 2.64E-08 & $9.28 \mathrm{E}-01$ & 2.45E-08 & $2.45 E-08$ & 120 & $1.32 E-01$ \\
\hline 28 & $\mathrm{Pu}$ & 242 & $2.64 E-08$ & 4.38E-04 & 1.16E-11 & & & \\
\hline 29 & $\mathrm{Sr}$ & 90 & $0.00 E+00$ & & & & & \\
\hline 30 & TTc & 99 & $2.24 \mathrm{E}-03$ & & & & & \\
\hline 31 & Th & 232 & $1.04 \mathrm{E}-07$ & & & & & \\
\hline 32 & $\mathrm{TI}$ & 204 & $0.00 \mathrm{E}+00$ & & & & & \\
\hline 33 & u & 235 & $3.99 E-06$ & & & & & \\
\hline 34 & $\mathrm{U}$ & 238 & 3.99E-06 & & & & & \\
\hline
\end{tabular}









\section{Attachment $\mathbf{P}$}

External contamination of grain during harvest spreadsheet calculations 


\begin{tabular}{|c|c|c|c|c|c|c|c|c|}
\hline & $\mathbf{A}$ & B & $\mathrm{H}$ & 1 & $\mathrm{~J}$ & $\bar{K}$ & L & $\mathbf{M}$ \\
\hline 2 & \multicolumn{8}{|c|}{ Grain-soil adhesion } \\
\hline 3 & & & & & & Group & Group DIL & Total \\
\hline 4 & & & & & & sum & & Dep for \\
\hline 5 & & & & & & & $\mathrm{~Bq} / \mathrm{kg}$ & DIL \\
\hline 6 & & & $\mathrm{~m} 2 / \mathrm{kg}$ & Unit & $T \times D E P$ & & & Cirm2 \\
\hline 7 & & & & Release & Ci/kg & $\mathrm{Ci} / \mathrm{kg}$ & & \\
\hline 8 & Element & At. No. & $T=D x F / 280$ & Fraction & & & & \\
\hline 9 & & & & $\mathrm{Ci} / \mathrm{m} 2$ & & & & \\
\hline 10 & Am & 241 & 1.22857E-05 & 1.87E-02 & 2.30E-07 & & & \\
\hline 11 & Am & 243 & 1.22857E-05 & & & & & \\
\hline 12 & $\mathrm{Ba}$ & 133 & 1.22857E-05 & & & & & \\
\hline 13 & $\mathrm{Ce}$ & 144 & 1.22857E-05 & & & & & \\
\hline 14 & Cf & 249 & 1.22857E-05 & & & & & \\
\hline 15 & Cf & 252 & 1.22857E-05 & & Am241 & & & \\
\hline 16 & $\mathrm{Cm}$ & 244 & 1.22857E-05 & & Pu238 & $7.98 E-07$ & 2 & 6.78E-05 \\
\hline 17 & $\mathrm{Cm}$ & 246 & 1.22857E-05 & & Pu239 & & & \\
\hline 18 & Co & 60 & 1.22857E-05 & & & & & \\
\hline 19 & Cs & 137 & 1.22857E-05 & & & & & \\
\hline 20 & $\mathrm{H}$ & 3 & 1.22857E-05 & & & & & \\
\hline 21 & Np & 237 & 1.22857E-05 & & & & & \\
\hline 22 & $\mathrm{Pm}$ & 147 & 1.22857E-05 & & & & & \\
\hline 23 & $\mathrm{Pu}$ & 238 & 1.22857E-05 & 4.61E-02 & 5.66E-07 & & & \\
\hline 24 & $\mathrm{Pu}$ & 239 & 1.22857E-05 & $1.20 E-04$ & 1.48E-09 & & & \\
\hline 25 & $\mathrm{Pu}$ & $239 / 241$ & 1.22857E-05 & & & & & \\
\hline 26 & $\mathrm{Pu}$ & 240 & 1.22857E-05 & $6.92 E-03$ & 8.50E-08 & & & \\
\hline 27 & $\mathrm{Pu}$ & 241 & 1.22857E-05 & $9.28 \mathrm{E}-01$ & 1.14E-05 & 1.14E-05 & 120 & 2.85E-04 \\
\hline 28 & $\mathrm{Pu}$ & 242 & 1.22857E-05 & 4.38E-04 & $5.38 E-09$ & & & \\
\hline 29 & $\mathrm{sr}$ & 90 & 1.22857E-05 & & & & & \\
\hline 30 & Tc & 99 & 1.22857E-05 & & & & & \\
\hline 31 & Th & 232 & $1.22857 \mathrm{E}-05$ & & & & & \\
\hline 32 & $\mathrm{TI}$ & 204 & 1.22857E-05 & & & & & \\
\hline 33 & $\mathrm{u}$ & 235 & 1.22857E-05 & & & & & \\
\hline 34 & u & 238 & 1.22857E-05 & & & & & \\
\hline
\end{tabular}




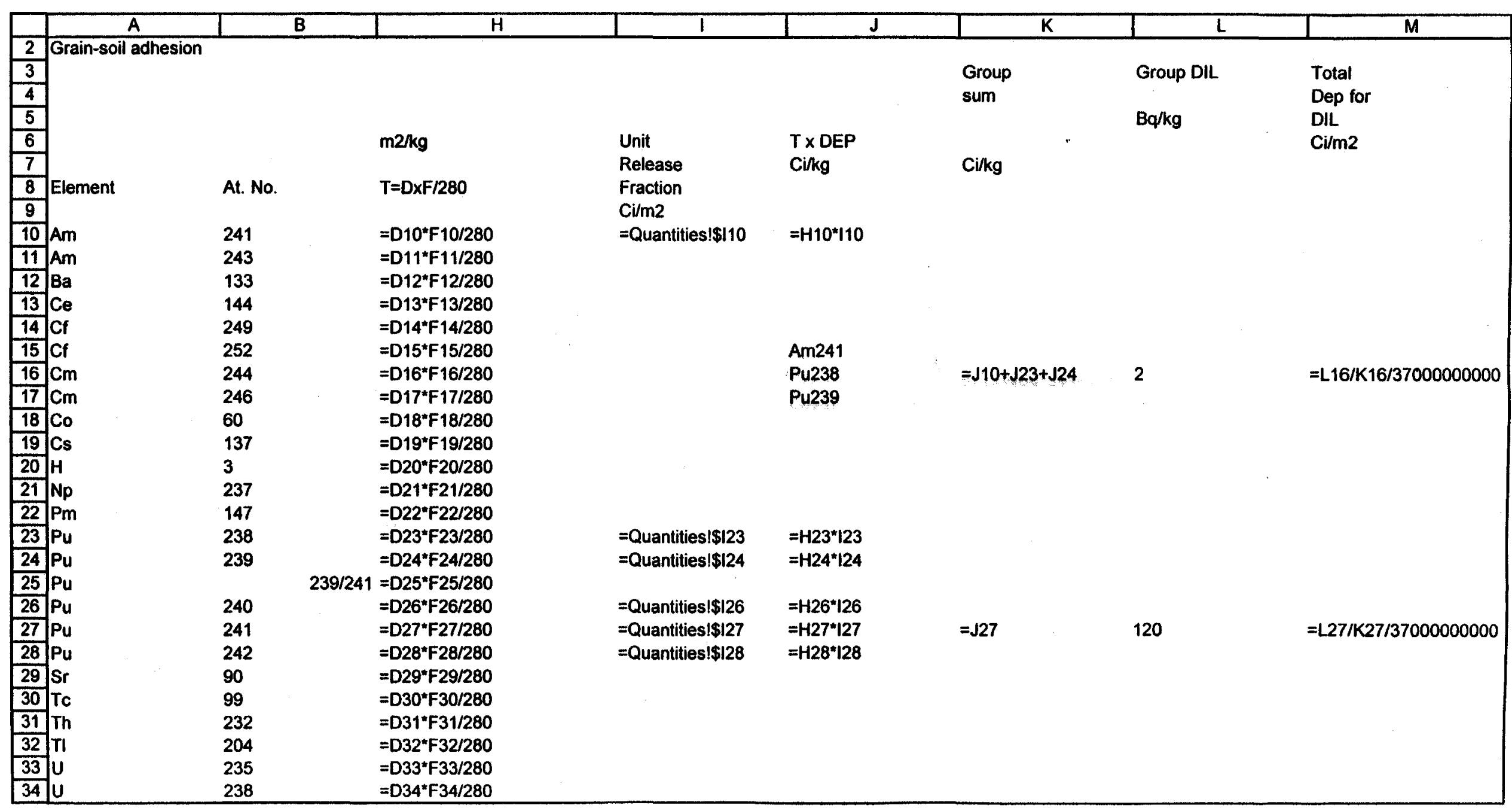


Attachment Q

Contamination of beverages spreadsheet calculations 


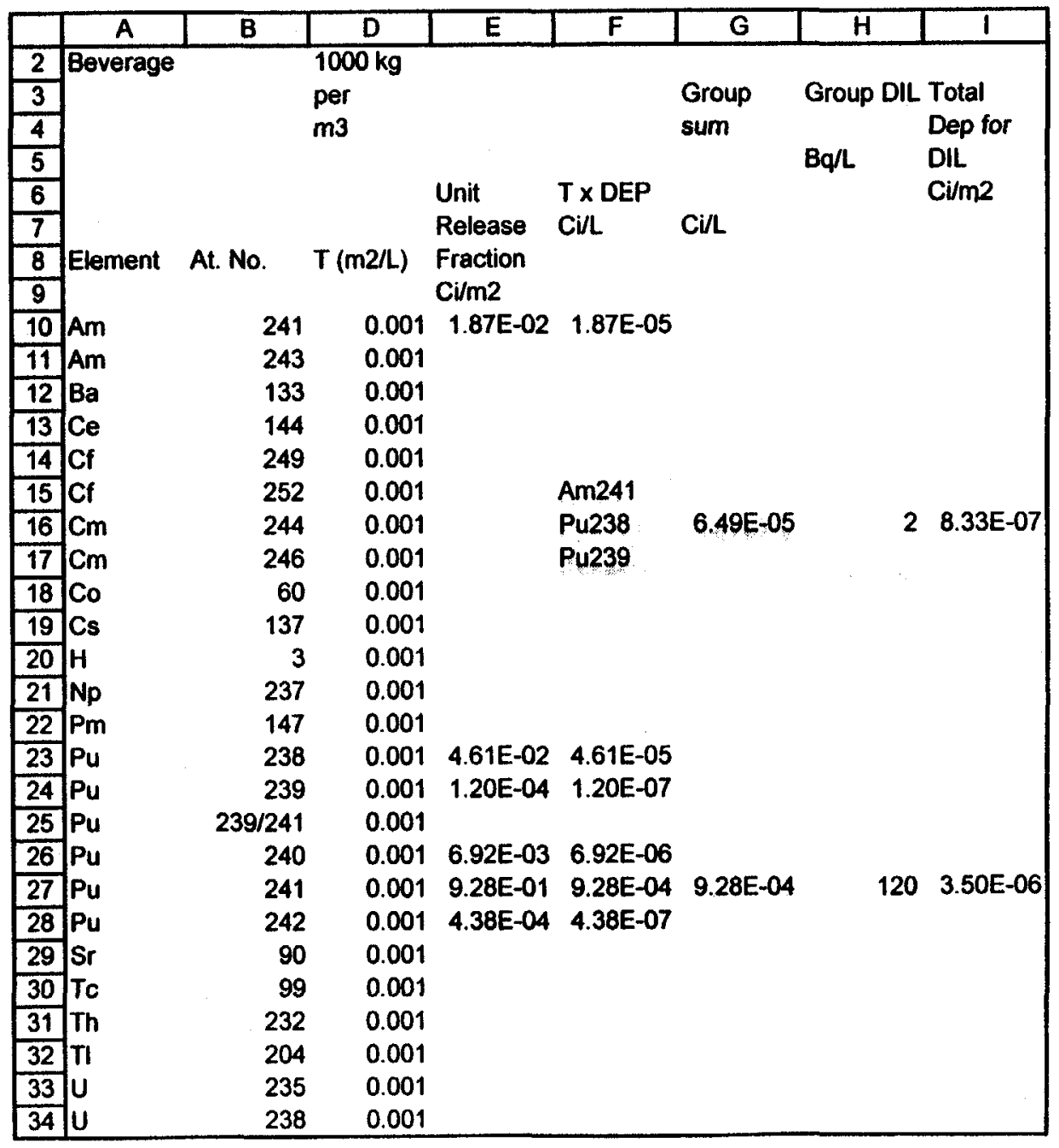




\begin{tabular}{|c|c|c|c|c|c|c|c|c|}
\hline & $A$ & B & $\bar{D}$ & $E$ & $F$ & $\mathbf{G}$ & $\mathrm{H}$ & 1 \\
\hline 2 & Beverage & & $1000 \mathrm{~kg}$ & & & & & \\
\hline 3 & & & per & & & Group & Group DIL & Total \\
\hline 4 & & & m3 & & & sum & & Dep for \\
\hline 5 & & & & & & & $\mathrm{~Bq} / \mathrm{L}$ & DIL \\
\hline 6 & & & & Unit & $T \times D E P$ & & & $\mathrm{Ci} / \mathrm{m} 2$ \\
\hline 7 & & & & Release & $\mathrm{Ci} / \mathrm{L}$ & $\mathrm{Ci} / \mathrm{L}$ & & \\
\hline 8 & Element & At. No. & $T(m 2 / L)$ & Fraction & & & & \\
\hline 9 & & & & $\mathrm{Ci} / \mathrm{m} 2$ & & & & \\
\hline 10 & Am & 241 & $=1 / 1000$ & $=$ Quantities $\mid \$ 110$ & $=D 10^{*} E 10$ & & & \\
\hline 11 & Am & 243 & $=1 / 1000$ & & & & & \\
\hline 12 & $\mathrm{Ba}$ & 133 & $=1 / 1000$ & & & & & \\
\hline 13 & $\mathrm{Ce}$ & 144 & $=1 / 1000$ & & & & & \\
\hline 14 & $c f$ & 249 & $=1 / 1000$ & & & & & \\
\hline 15 & Cf & 252 & $=1 / 1000$ & & Am241 & & & \\
\hline 16 & $\mathrm{Cm}$ & 244 & $=1 / 1000$ & & Pu238 & $=F 10+F 23+F 24$ & 2 & $=H 16 / G 16 / 37000000000$ \\
\hline 17 & $\mathrm{Cm}$ & 246 & $=1 / 1000$ & & Pu239 & & & \\
\hline 18 & Co & 60 & $=1 / 1000$ & & & & & \\
\hline 19 & Cs & 137 & $=1 / 1000$ & & & & & \\
\hline 20 & $\mathbf{H}$ & 3 & $=1 / 1000$ & & & & & \\
\hline 21 & Np & 237 & $=1 / 1000$ & & & & & \\
\hline 22 & $\mathrm{Pm}$ & 147 & $=1 / 1000$ & & & & & \\
\hline 23 & $\mathrm{Pu}$ & 238 & $=1 / 1000$ & $=$ Quantities $1 \$ 123$ & $=D 23^{*} E 23$ & & & \\
\hline 24 & Pu & 239 & $=1 / 1000$ & $=$ Quantities $1 \$ 124$ & $=D 24^{*} E 24$ & & & \\
\hline 25 & $\mathrm{Pu}$ & & $=1 / 1000$ & & & & & \\
\hline 26 & $\mathrm{Pu}$ & 240 & $=1 / 1000$ & $=$ Quantities $1 \$ 126$ & $=D 26^{\star} E 26$ & & & \\
\hline 27 & Pu & 241 & $=1 / 1000$ & $=$ Quantities $\$ \$ 127$ & $=\mathrm{D} 27^{\star} \mathrm{E} 27$ & $=F 27$ & 120 & $=H 27 / G 27 / 37000000000$ \\
\hline 28 & $\mathrm{Pu}$ & 242 & $=1 / 1000$ & $=$ Quantities $1 \$ 128$ & $=028^{\star} E 28$ & & & \\
\hline 29 & $\mathrm{sr}$ & 90 & $=1 / 1000$ & & & & & \\
\hline 30 & $\mathrm{Tc}$ & 99 & $=1 / 1000$ & & & & & \\
\hline 31 & Th & 232 & $=1 / 1000$ & & & & & \\
\hline 32 & $\mathrm{TI}$ & 204 & $=1 / 1000$ & & & & & \\
\hline 33 & $u$ & 235 & $=1 / 1000$ & & & & & \\
\hline 34 & $u$ & 238 & $=1 / 1000$ & & & & & \\
\hline
\end{tabular}




\section{Attachment R}

HOTSPOT results - average meteorology, $0.1 \mathrm{~cm} \mathrm{~s}^{-1}$ 


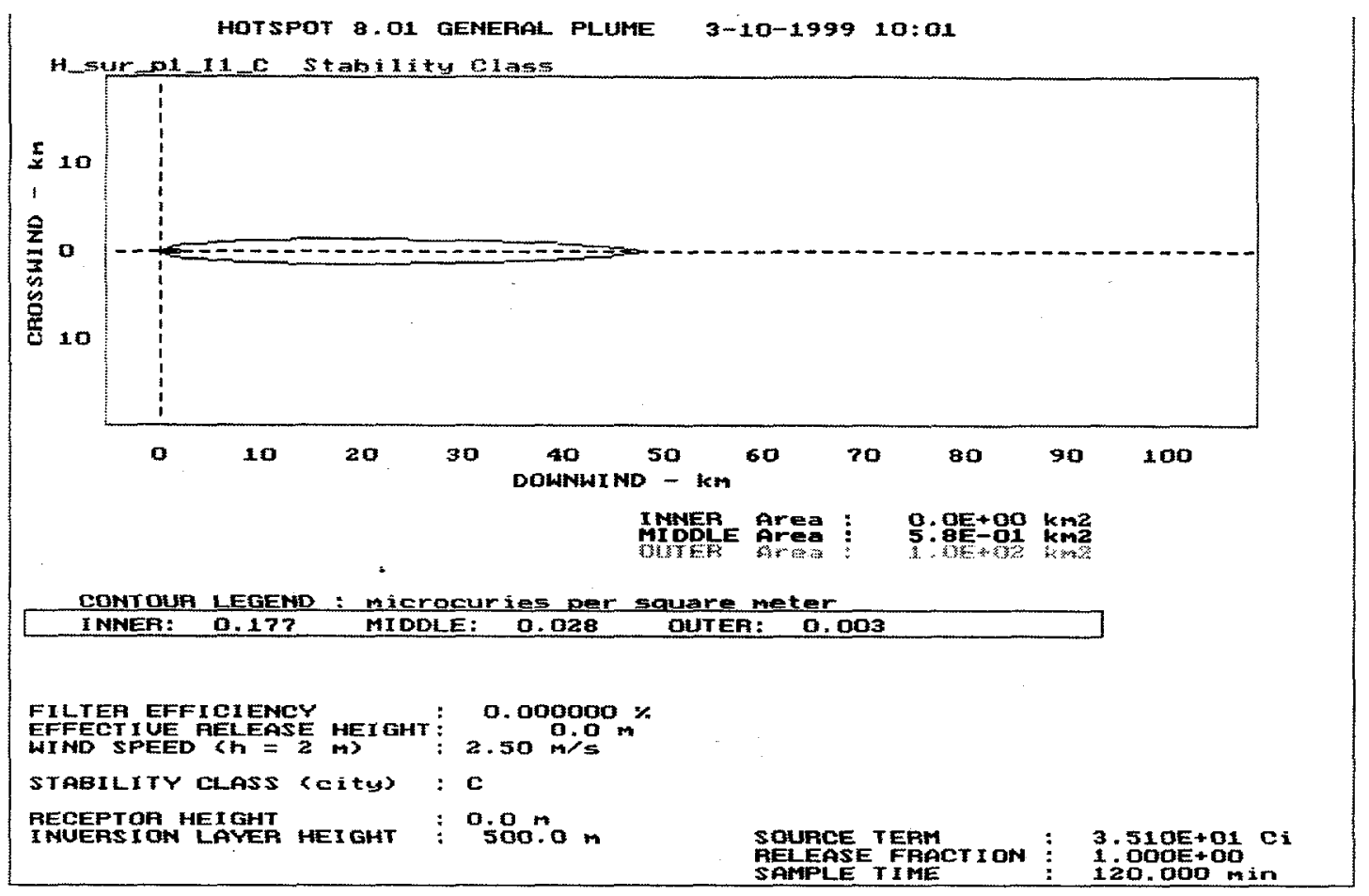

The $0.177 \mu \mathrm{Ci} \mathrm{m}^{-2}$ area value above is not used. It is erroneously displayed as zero because of the course grid spacing. This case was rerun to give more a more accurate area calculation.

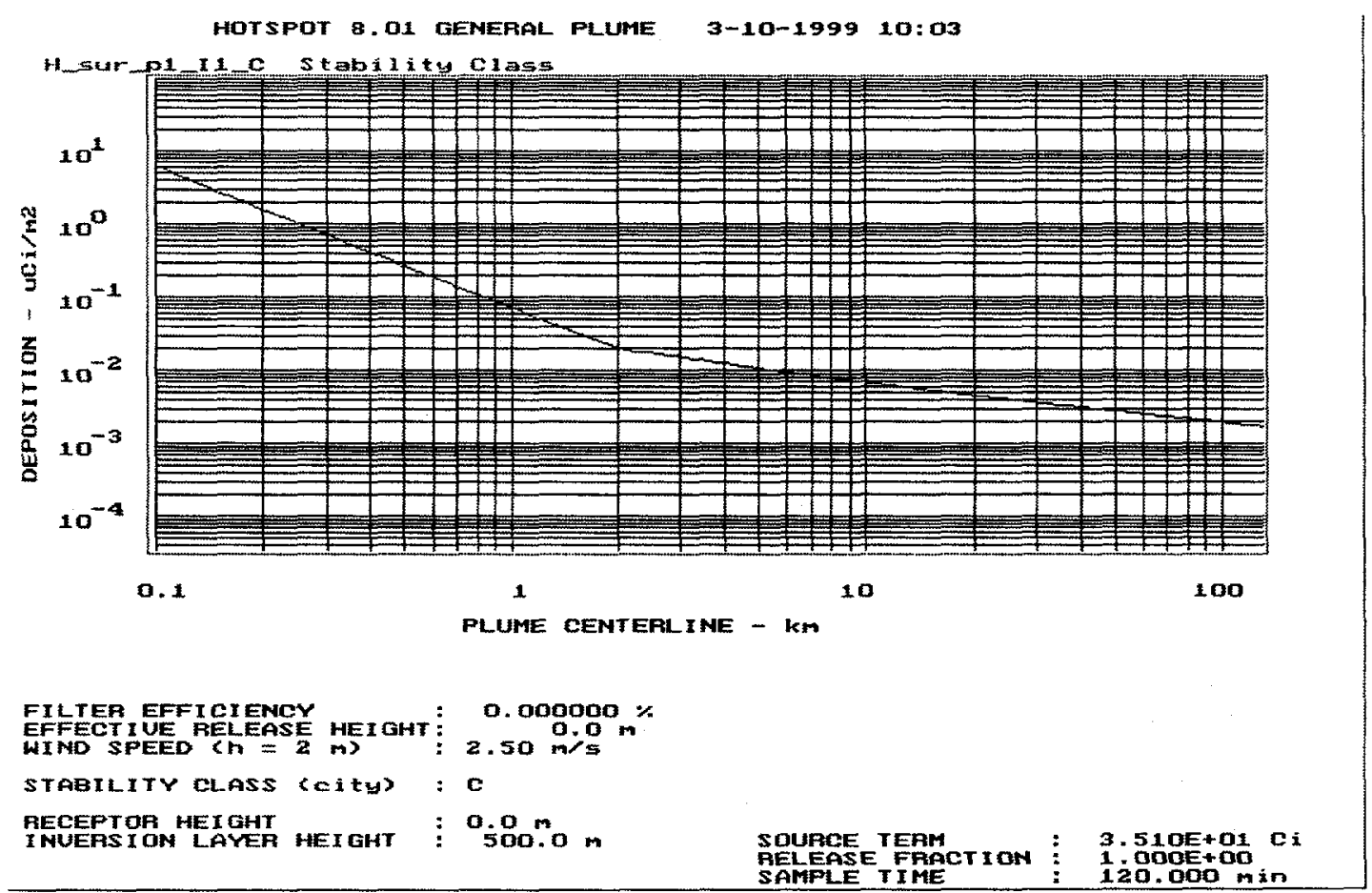




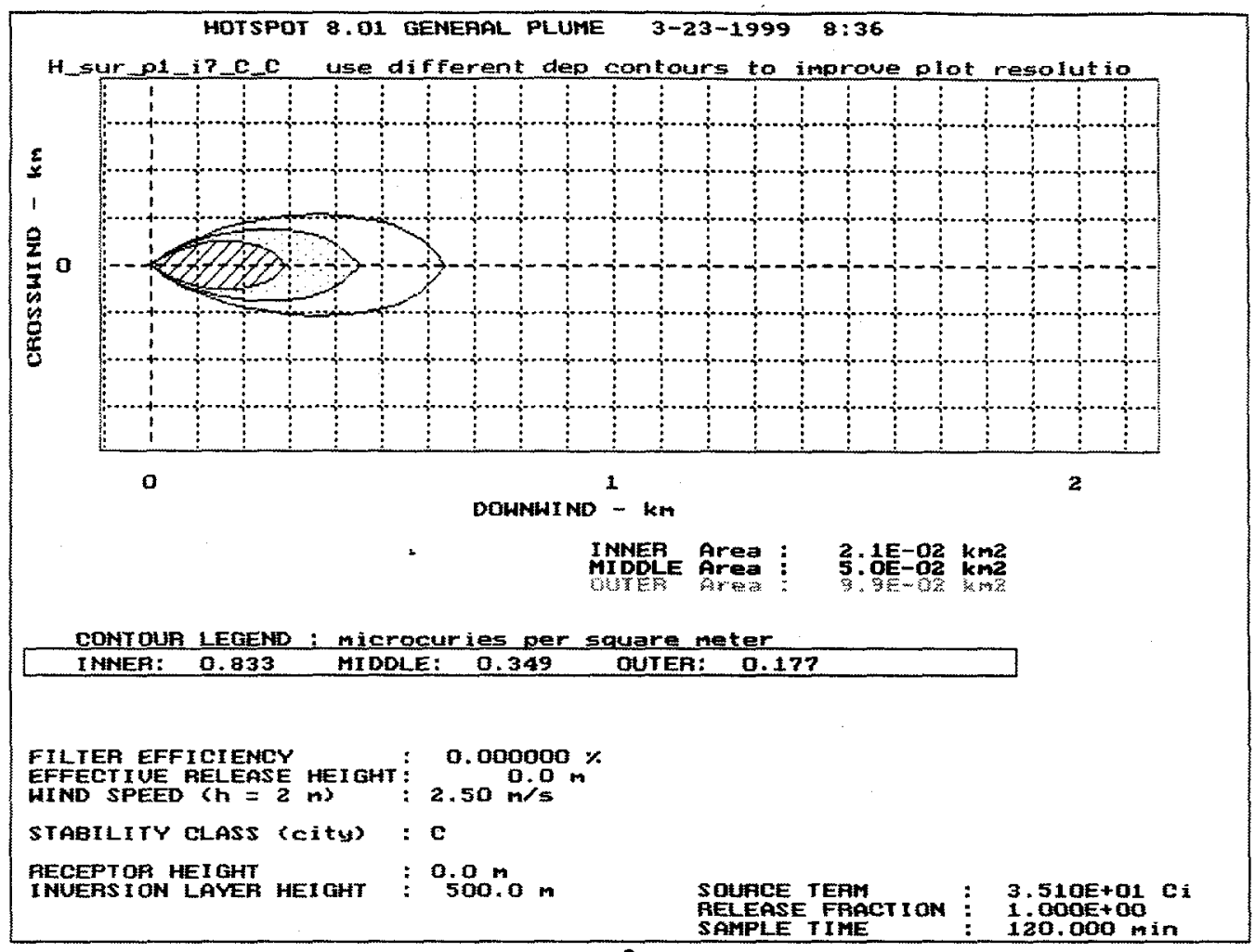

This plot was used for the $0.177 \mu \mathrm{Ci} \mathrm{m}^{-2}$ area value. 

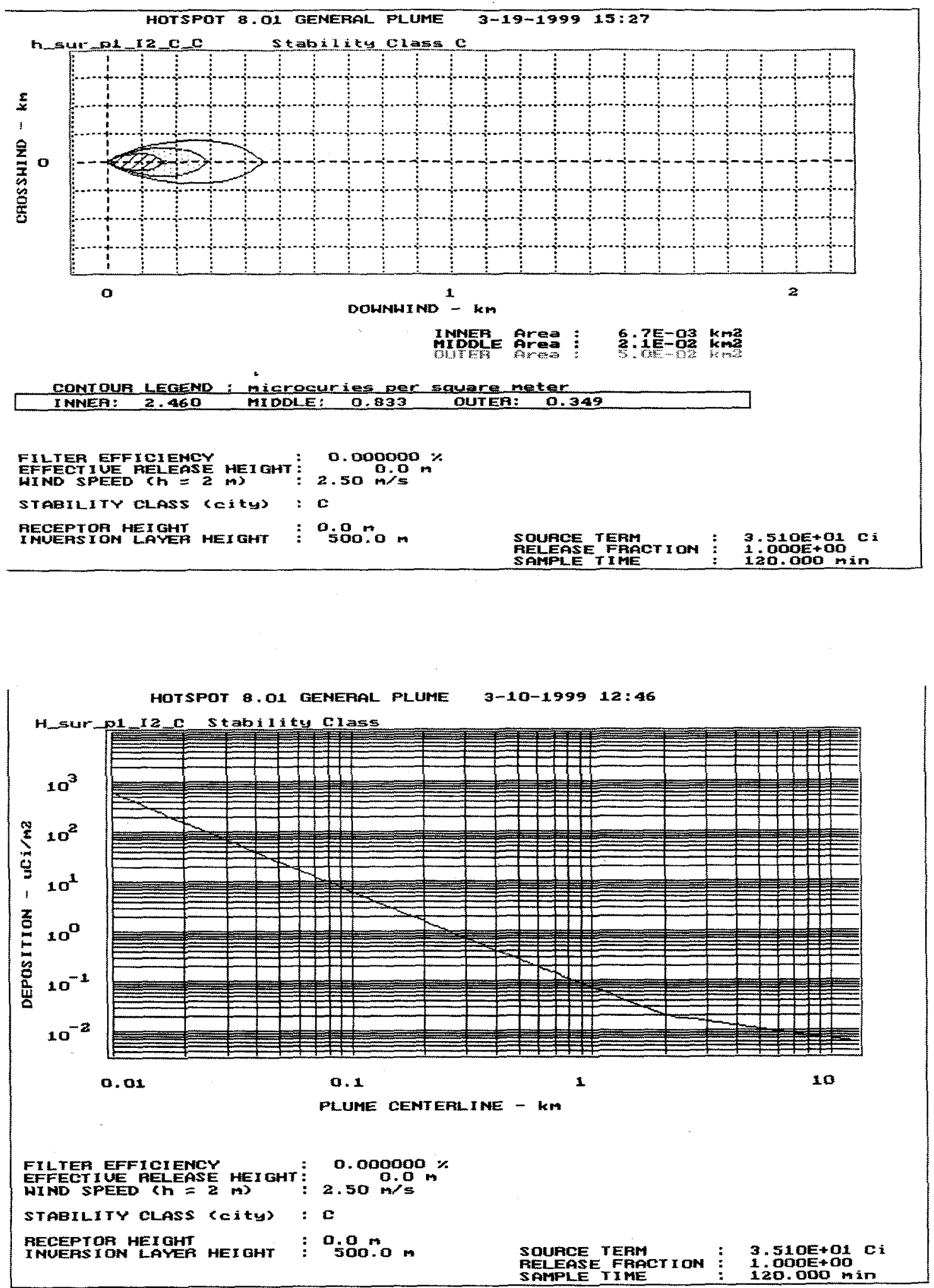


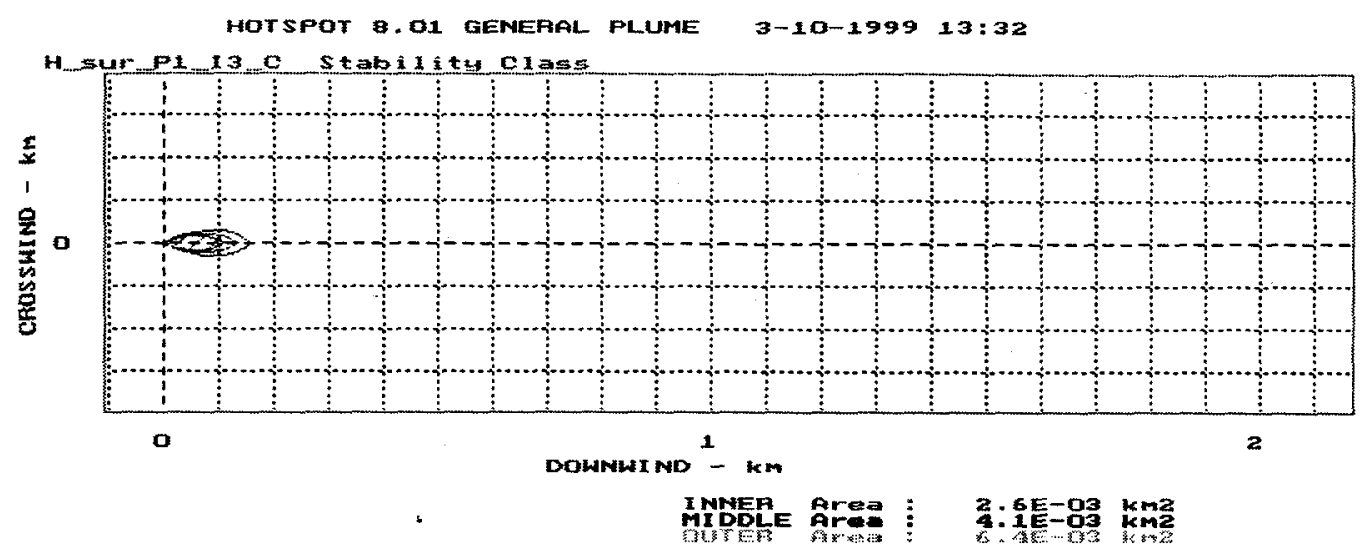

CONTOUR LEGEND: nicracuries per square neter

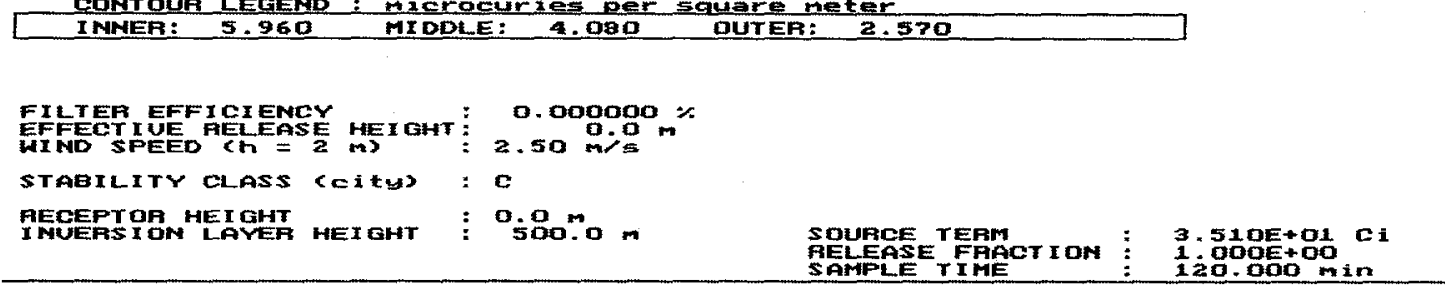

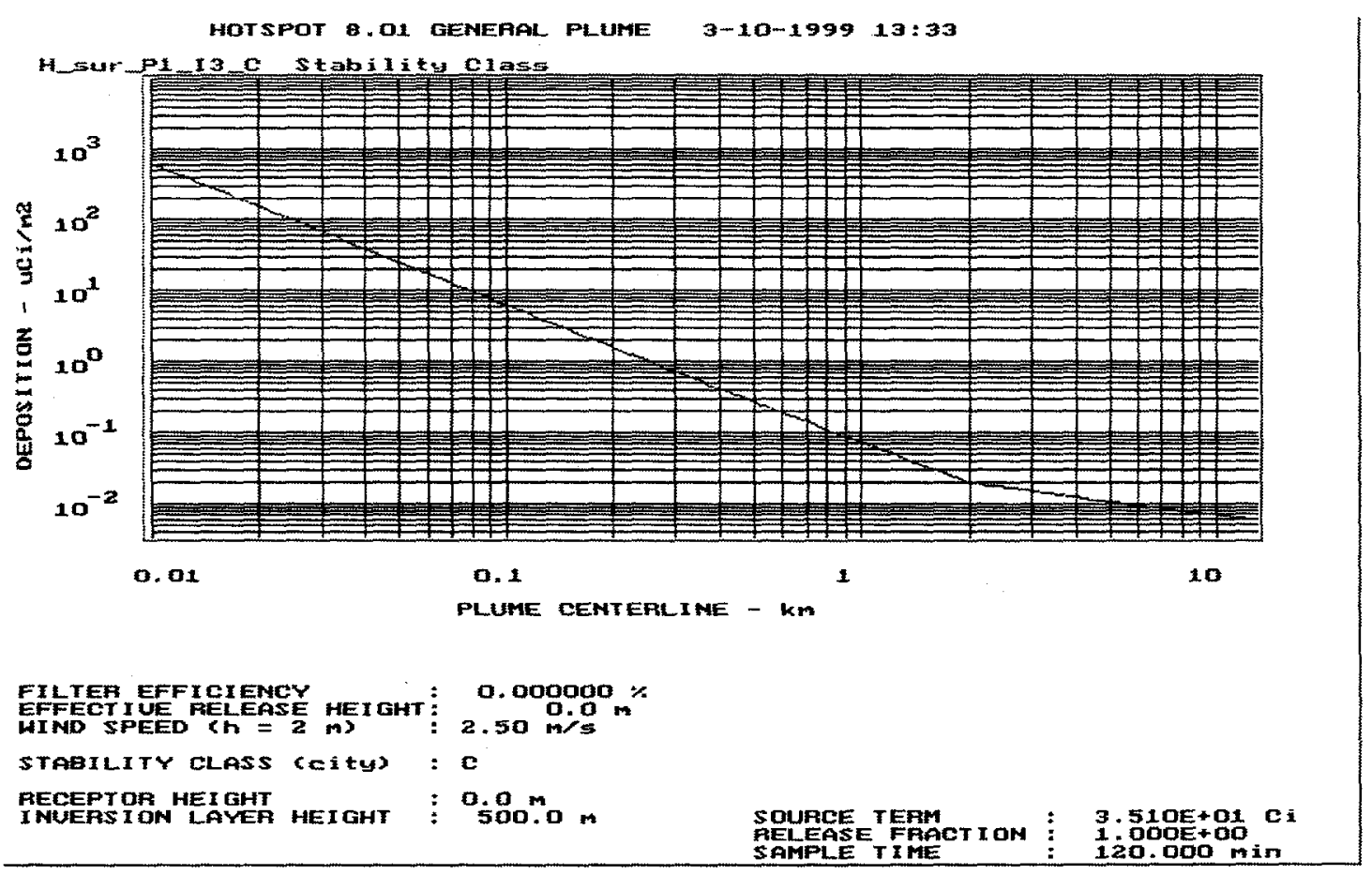




\section{Attachment S}

HOTSPOT results - average meteorology, $1.0 \mathrm{~cm} \mathrm{~s}^{-1}$ 

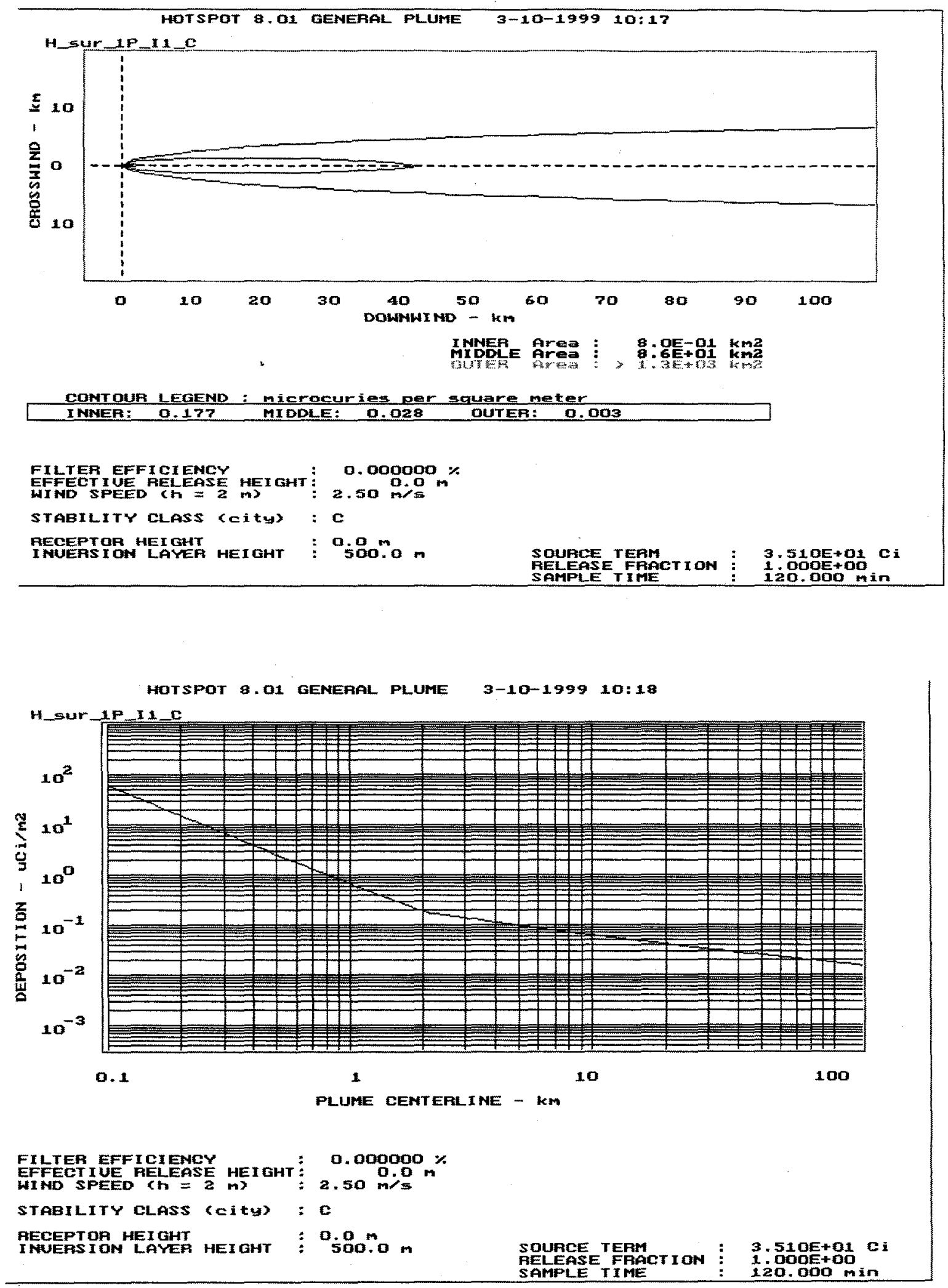

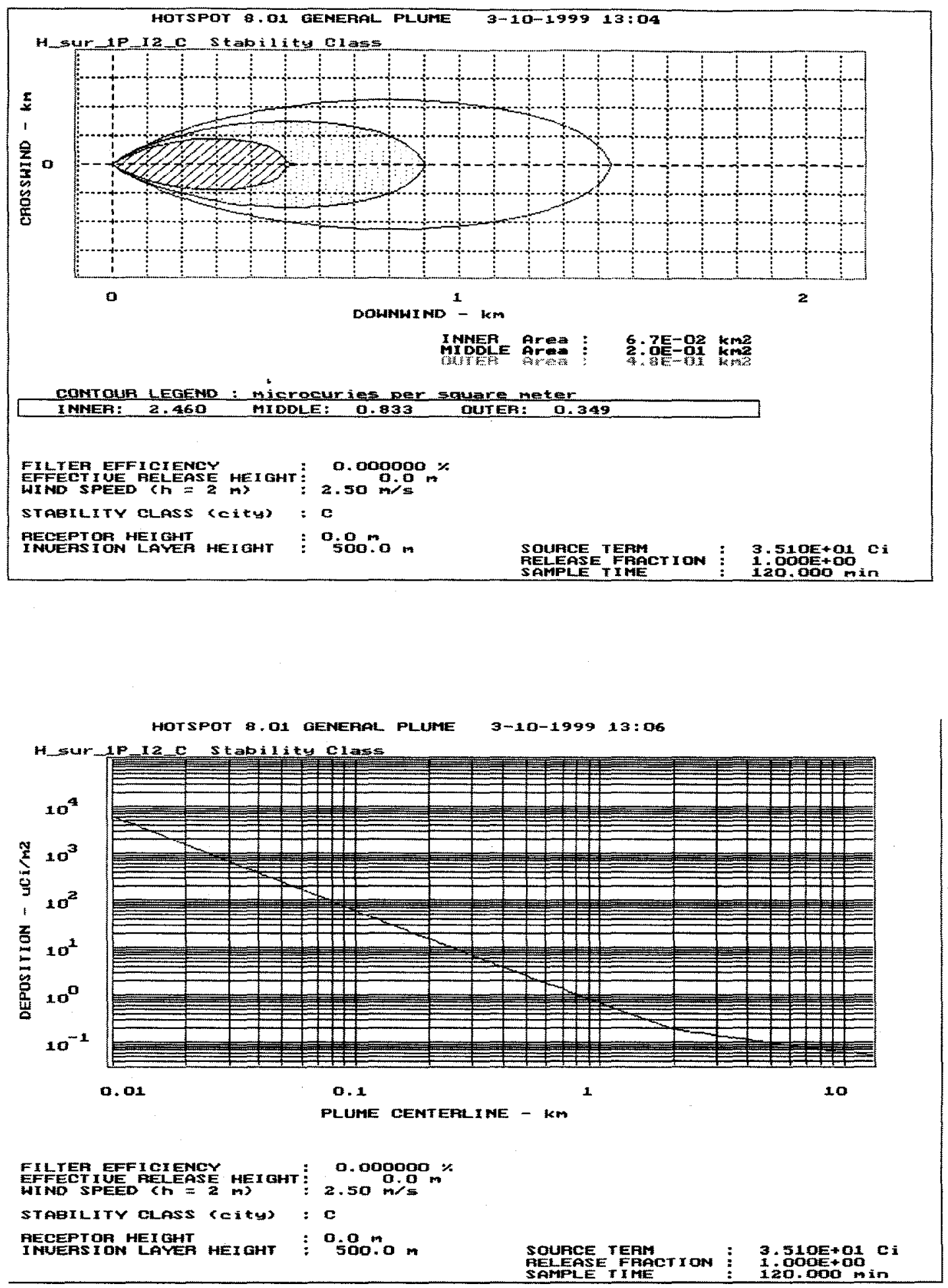

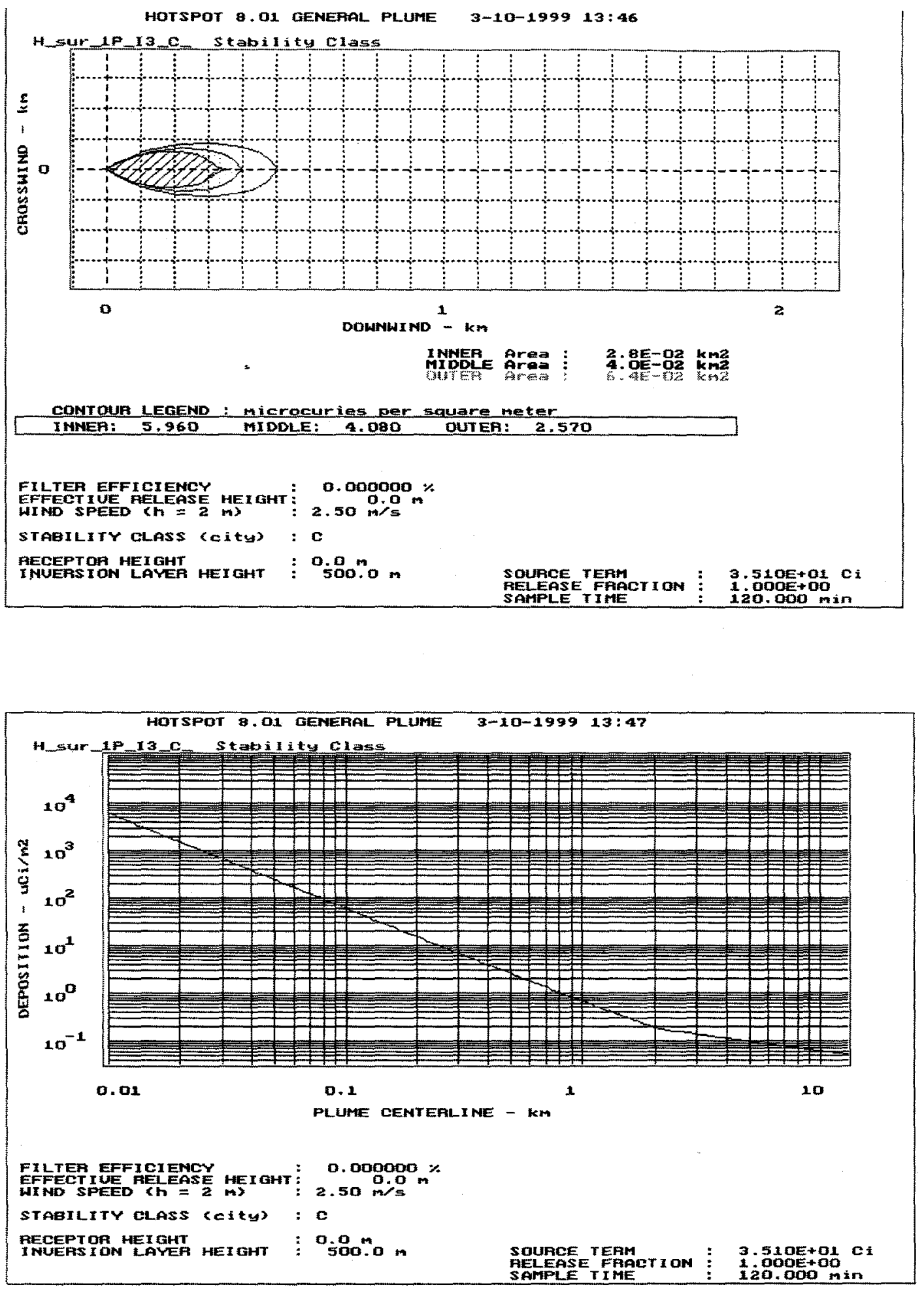




\section{Attachment T}

HOTSPOT results - average meteorology, $10 \mathrm{~cm} \mathrm{~s}^{-1}$ 



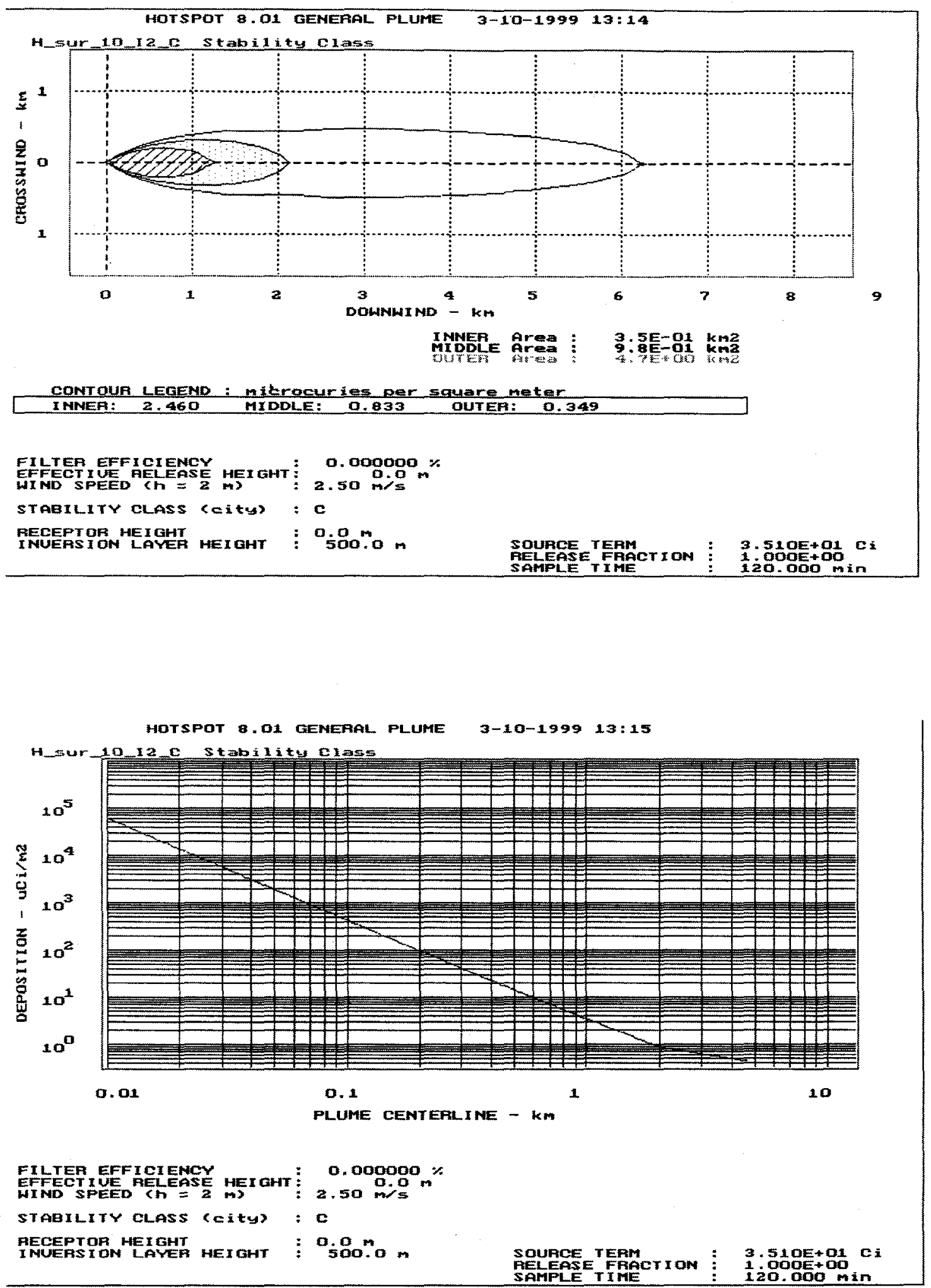

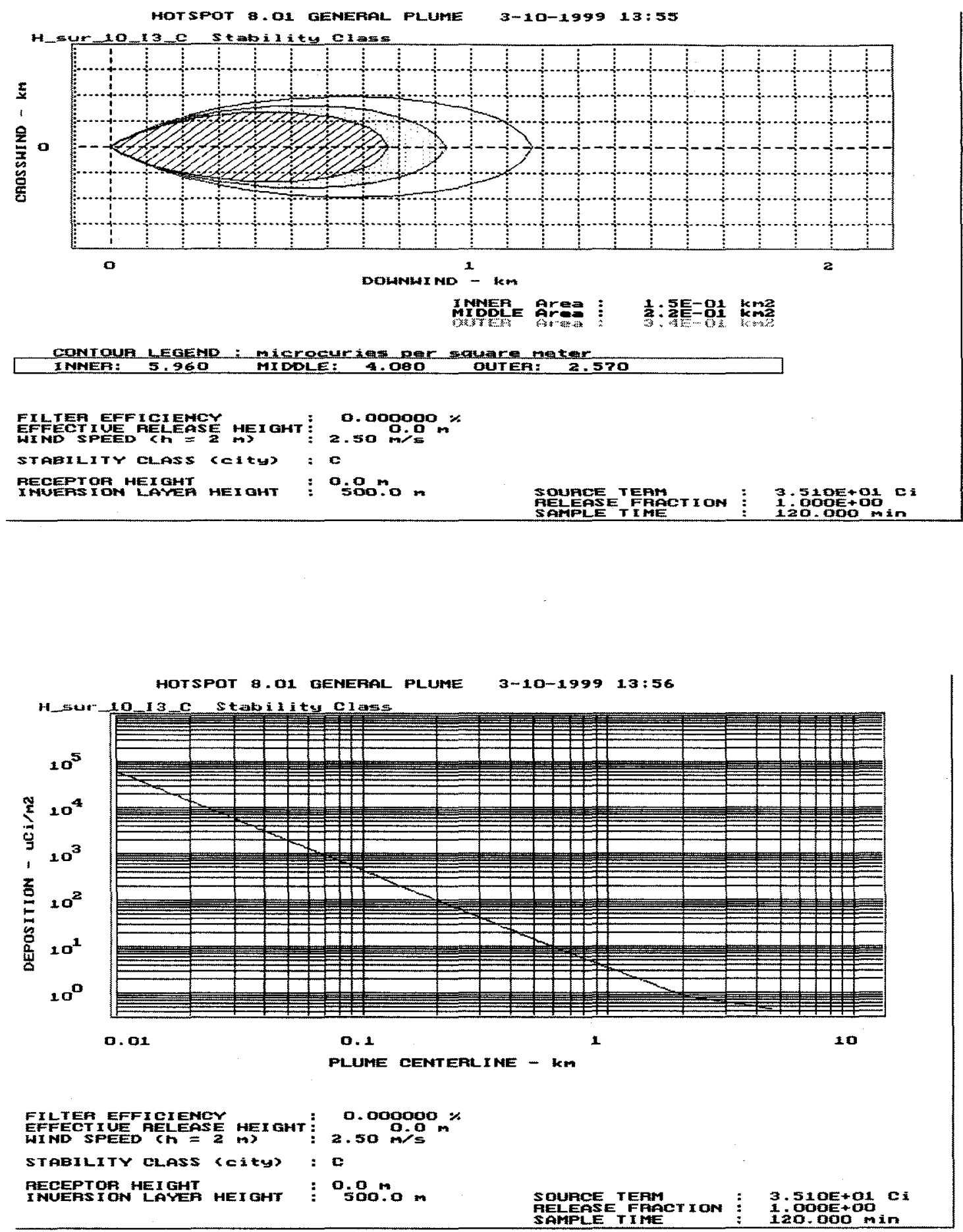




Ingestion Pathway Consequences of an H-Separations Coil and Tube Failure (U)

\section{Attachment $\mathbf{U}$}

HOTSPOT results - adverse meteorology, $0.1 \mathrm{~cm} \mathrm{~s}^{-1}$ 

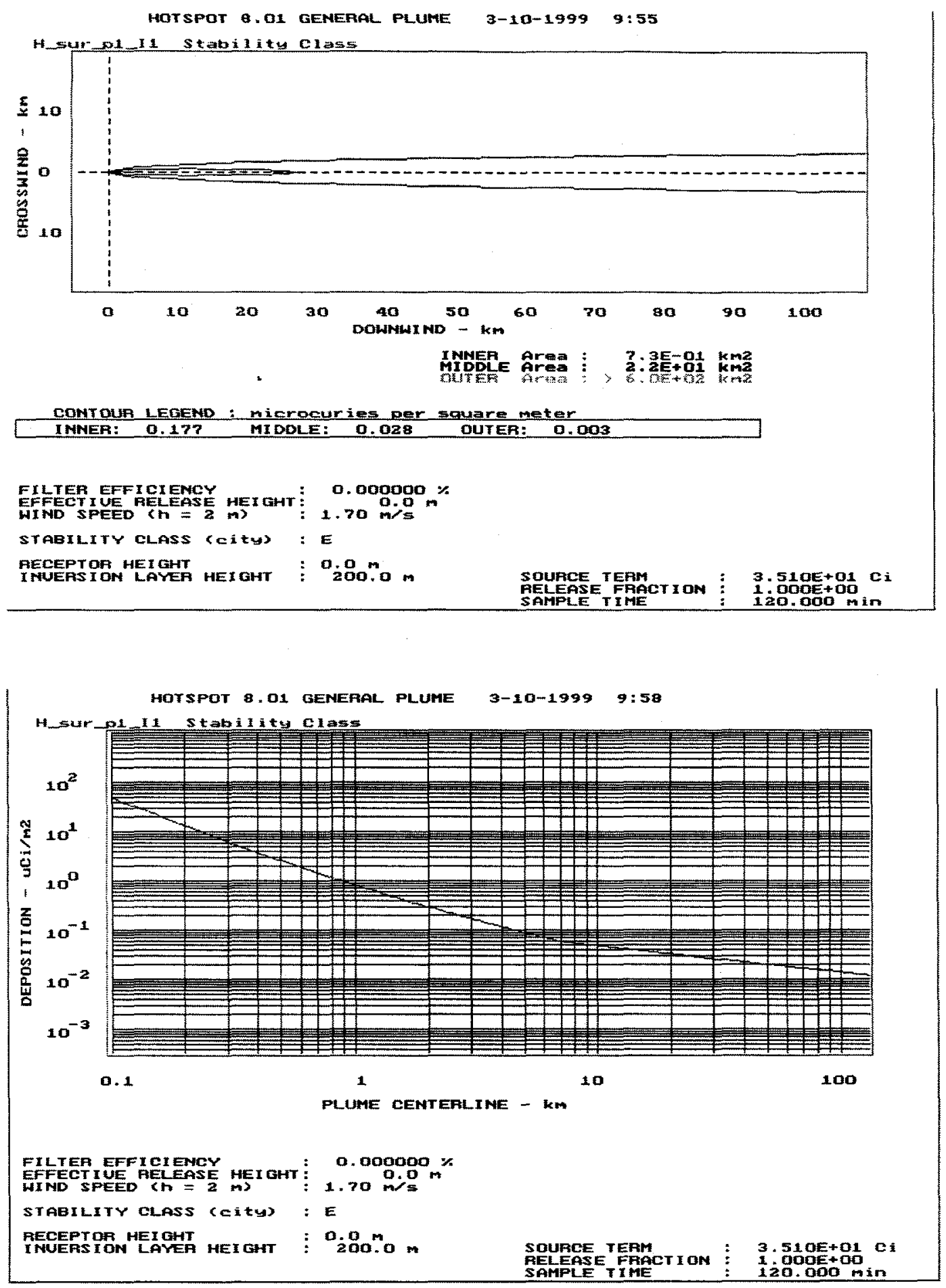

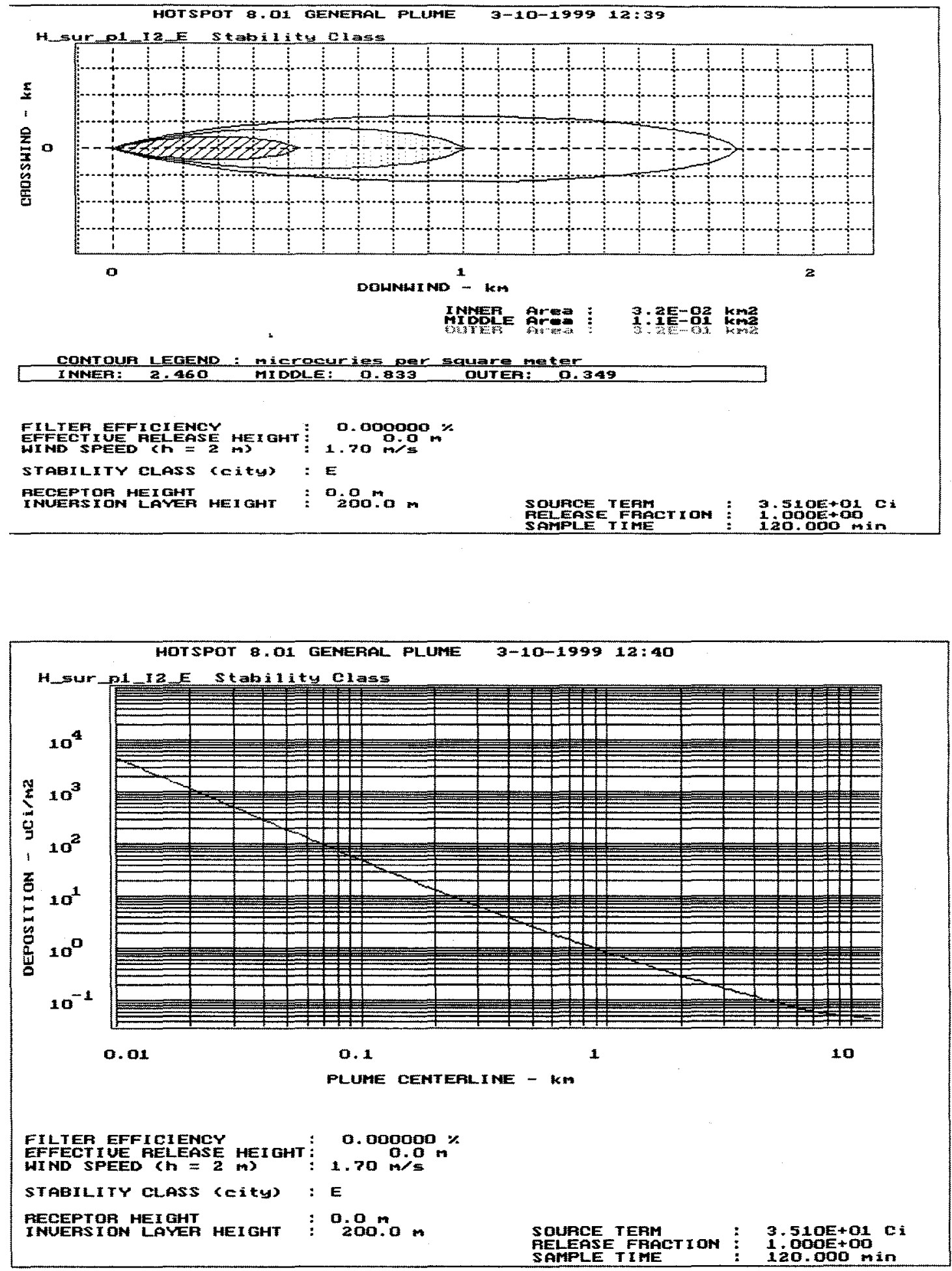

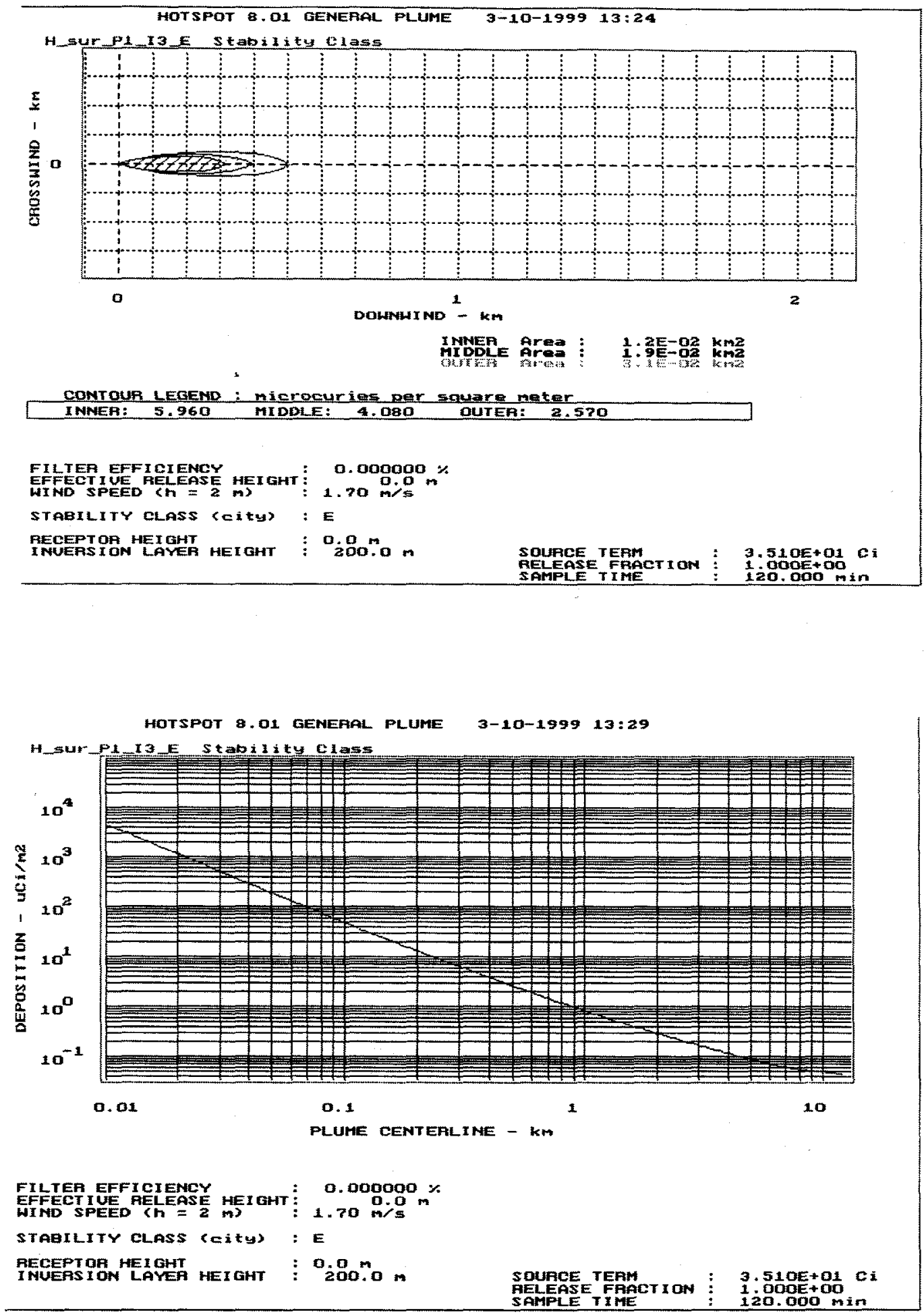

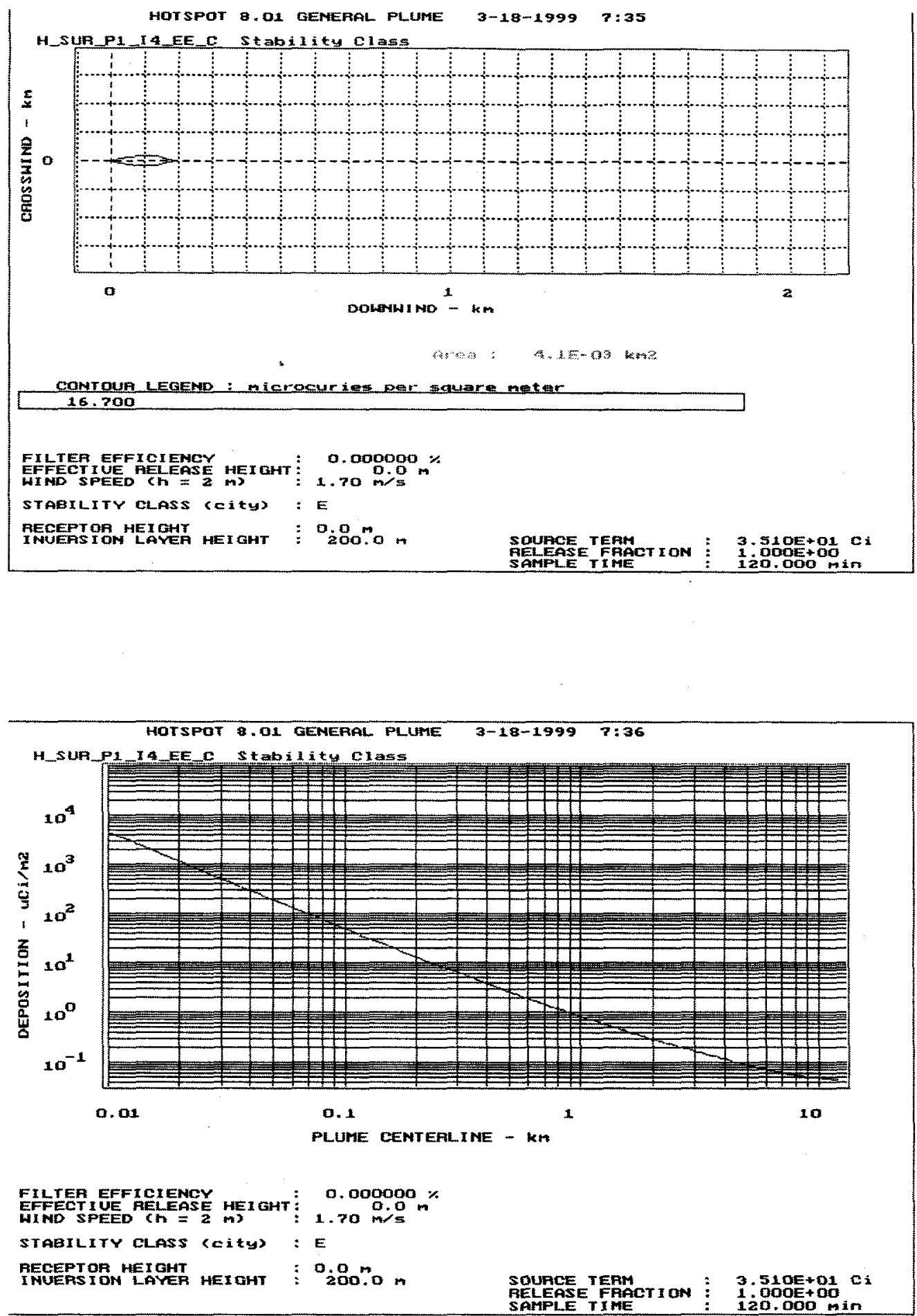


\section{Attachment $V$}

HOTSPOT results - adverse meteorology, $1.0 \mathrm{~cm} \mathrm{~s}^{-1}$ 



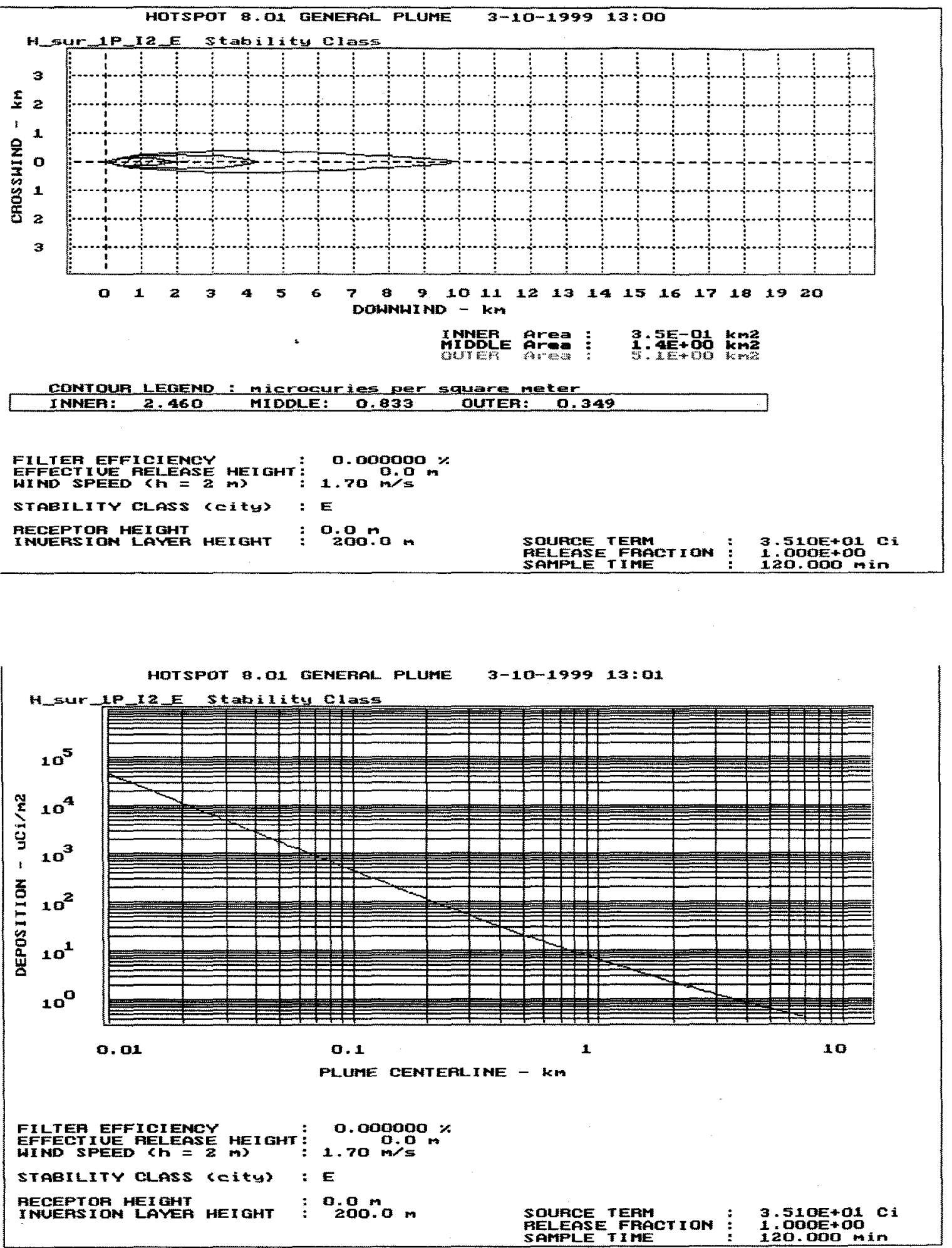

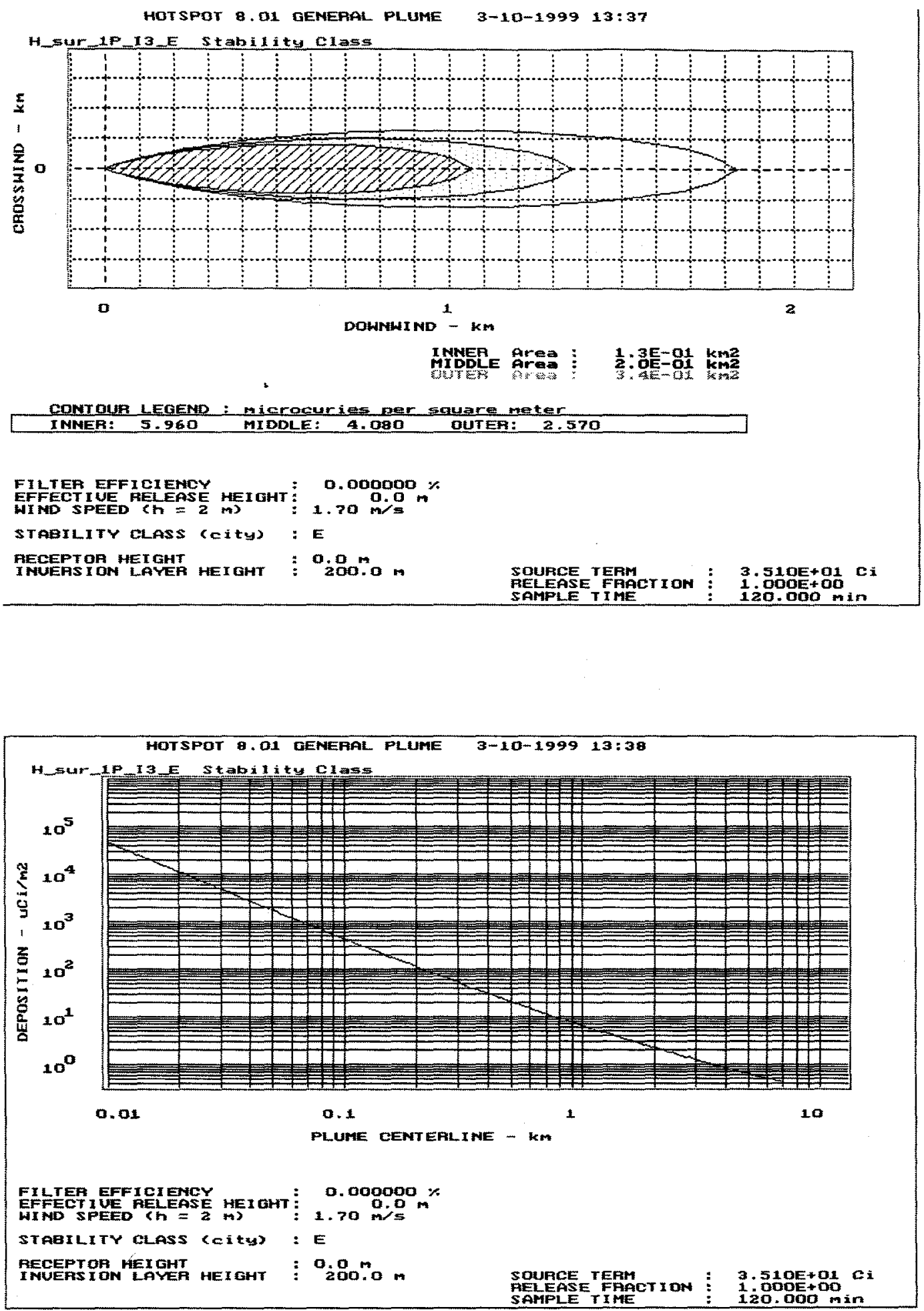






\section{Attachment W}

HOTSPOT results - adverse meteorology, $10 \mathrm{~cm} \mathrm{~s}^{-1}$ 



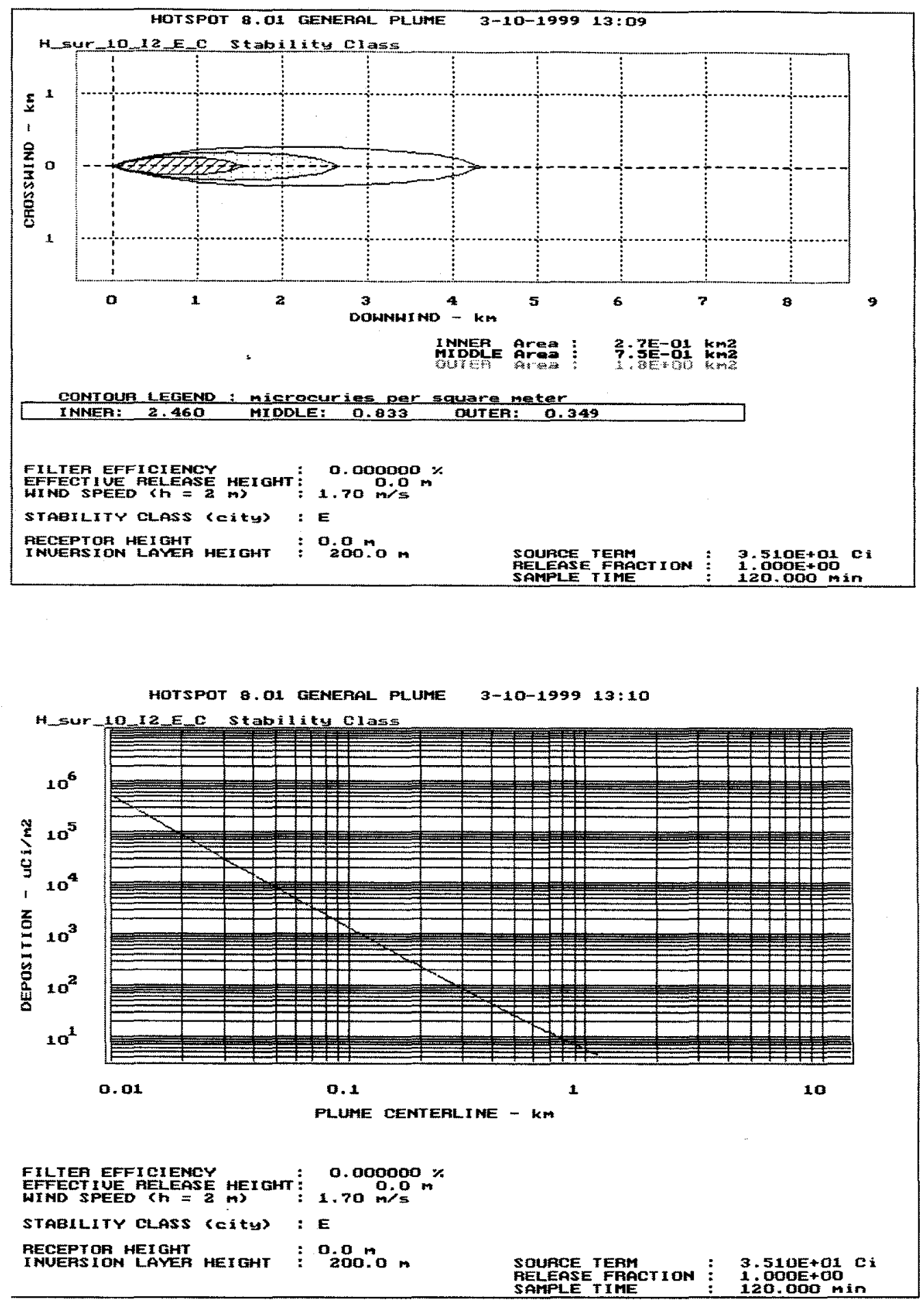

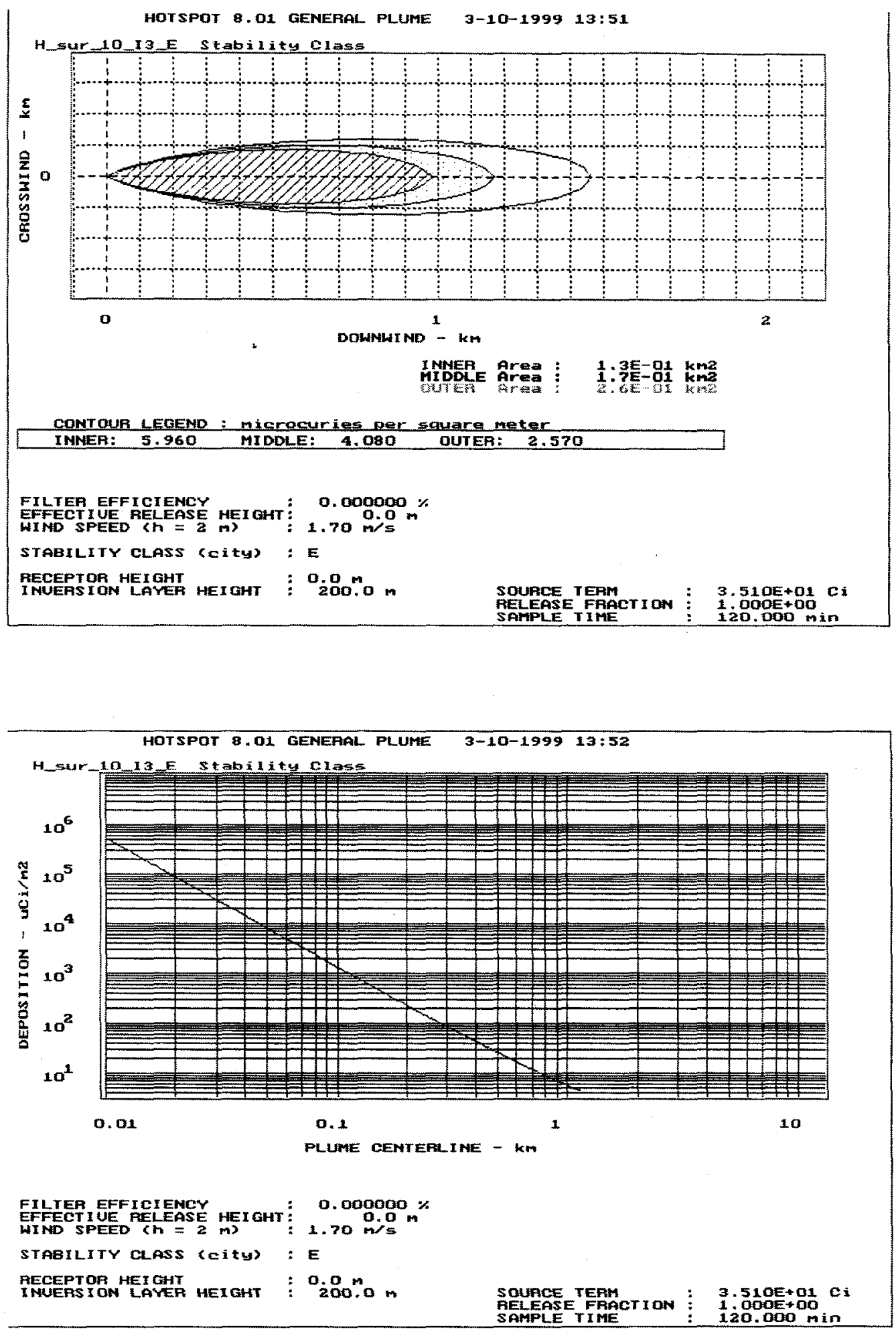




DISTRIBUTION FOR WSRC-TR-99-00089, Revision 0

WSMS Personnel:

1. Eric Shogren

2. Kevin O'Kula

CCC-3

3. Jay Thompson

CCC -3

4. Liz Thompson

CCC-3, Cube 125

5. Danielle Marx

$\mathrm{CCC}-3$

6. Thomas Brown

CCC-3

CCC-3

WSRC Personnel:

7. Davida Matthews

Building 706-8C

DOE Personnel:

1. Christina T. Edwards

P.O. Box A, Aiken, SC

Files:

1. WSRC Document Control (Original) c/o Debbie Strautmann

CCC-3, Cube 340 